\title{
Caloric curves of classical self-gravitating systems in general relativity
}

\author{
Giuseppe Alberti \\ Laboratoire de Physique Théorique, Université de Toulouse, CNRS, UPS, France and \\ Living Systems Research, Roseggerstraße 27/2, A-9020 Klagenfurt am Wörthersee, Austria \\ Pierre-Henri Chavanis \\ Laboratoire de Physique Théorique, Université de Toulouse, CNRS, UPS, France
}

\begin{abstract}
We determine the caloric curves of classical self-gravitating systems at statistical equilibrium in general relativity. In the classical limit, the caloric curves of a self-gravitating gas depend on a unique parameter $\nu=G N m / R c^{2}$, called the compactness parameter, where $N$ is the particle number and $R$ the system's size. Typically, the caloric curves have the form of a double spiral. The "cold spiral", corresponding to weakly relativistic configurations, is a generalization of the caloric curve of nonrelativistic classical self-gravitating systems. The "hot spiral", corresponding to strongly relativistic configurations, is similar (but not identical) to the caloric curve of the ultrarelativistic self-gravitating black-body radiation. We introduce two types of normalization of energy and temperature in order to obtain asymptotic caloric curves describing respectively the cold and the hot spirals in the limit $\nu \rightarrow 0$. As the number of particles increases, the cold and the hot spirals approach each other, merge at $\nu_{S}^{\prime}=0.128$, form a loop above $\nu_{S}=0.1415$, reduce to a point at $\nu_{\max }=0.1764$, and finally disappear. Therefore, the double spiral shrinks when the compactness parameter $\nu$ increases, implying that general relativistic effects render the system more unstable. We discuss the nature of the gravitational collapse at low and high energies with respect to a dynamical (fast) or a thermodynamical (slow) instability.

PACS numbers: 04.40.Dg, 05.70.-a, 05.70.Fh, 95.30.Sf, 95.35.+d
\end{abstract}

\section{INTRODUCTION}

The statistical mechanics of self-gravitating systems has a rich history. In this introduction (see also [1]), we review the main developments of this subject, restricting ourselves to classical self-gravitating systems (the case of self-gravitating fermions is reviewed in [2, 3] and the case of self-gravitating bosons is reviewed in [4]). This review is useful because no history of this important topic has been given previously. We successively consider nonrelativistic stellar systems and general relativistic star clusters.

The statistical mechanics of nonrelativistic stellar systems started with the work of Chandrasekhar [5] (see Appendix A for the pre-history of the subject). He developed a kinetic theory of stellar systems in order to determine their time of relaxation due to gravitational twobody close encounters. He obtained an expression of the form $t_{\text {coll }} \sim(N / \ln N) t_{\mathrm{D}}$, where $N$ is the number of stars in the system and $t_{\mathrm{D}}$ is the dynamical time. ${ }^{1}$ For galaxies, containing a large number of stars $\left(N \sim 10^{12}\right)$, the relaxation time is much larger than the age of the Universe so these systems are essentially collisionless, described by the Vlasov (or collisionless Boltzmann) equation. ${ }^{2}$ By

\footnotetext{
${ }^{1}$ An estimate of the time of relaxation of a star cluster was previously obtained by Jeans [6] 9, Eddington [10, Charlier [1], Schwarzschild [12, Rosseland 13], Smart [14, Ambartsumian 15, Mineur 16, and Spitzer 17.

2 This equation was introduced by Jeans [18 in the context of stellar systems and by Vlasov [19] for Coulombian plasmas. See
}

contrast, for globular clusters containing a small number of stars $\left(N \sim 10^{6}\right)$, the time of relaxation is of the order of their age so they should be close to a state of statistical equilibrium. ${ }^{3}$ Statistical equilibrium may also be established in the central regions of galaxies where the density is high. Therefore, Chandrasekhar [5] assumed that globular clusters (and the core of galaxies) are in a statistical equilibrium state described by the MaxwellBoltzmann distribution in which the gravitational potential is determined by the Poisson equation. This leads to the Boltzmann-Poisson equation which is equivalent to Emden's equation describing the hydrostatic equilibrium of an isothermal gas sphere 21, 22. However, Chandrasekhar 5 noted that the isothermal approximation cannot be used for a complete description of a globular cluster because it predicts an infinite mass. ${ }^{4}$ Indeed, the density of a self-gravitating isothermal sphere decreases as $r^{-2}$ at large distances 27, 28. Therefore, the isothermal approximation is inadequate for describing the outer regions of a globular cluster. Statistical equilibrium does not hold anymore at low densities because the relaxation time increases so the system does not reach a statistical equilibrium state. On the other hand, there is a contin-

Hénon [20] for some comments about the name that should be given to this equation.

3 The important difference between the relaxation time of galaxies and globular clusters was first pointed out by Jeans [6].

4 This problem was previously pointed out by Eddington [23, 24], Jeans [7, Heckmann and Siedentopf [25], Dicke [26], and Spitzer 17. 
ual loss of stars by escape and this phenomenon is important in the outer regions (stars with an energy larger than the escape energy leave the cluster). The continuous loss of stars from the system leads to a contraction and to a gradual disintegration of the system as pointed out by Jeans [7, Ambartsumian [15, Dicke [26, and Spitzer [17. In this sense, there is no statistical equilibrium state for self-gravitating systems. However, evaporation occurs on a long timescale, much larger than the relaxation time. Therefore, the escape of stars is a slow process and the system can reach a quasiequilibrium state, close to the Maxwell-Boltzmann distribution, on an intermediate timescale. The effect of evaporation is to change the Maxwell-Boltzmann distribution close to the escape energy. ${ }^{5}$ Using an analogy between stellar dynamics and Brownian motion, Chandrasekhar $32 \sqrt{34}$ derived a kinetic equation for stellar systems of the Fokker-Planck form ${ }^{6}$ and used it to study in greater detail the relaxation of the system towards statistical equilibrium and the escape of stars from globular clusters (see also White [36, Spitzer and Härm 37, von Hoerner [38, King 31, 3944, Hénon [45]48, Michie 30, 49] 51], Miller and Parker [52, and Spitzer and Saslaw [53]). King [40] suggested that a globular cluster, as a result of evaporation and contraction, may end as a binary star.

Ogorodnikov [54, 55] looked for the most probable distribution of stars in a galaxy by using methods of statistical mechanics. This amounts to maximizing the Boltzmann entropy at fixed energy and particle number. ${ }^{7}$ He only considered the first order variational problem (extremization of entropy) and derived the mean field Maxwell-Boltzmann distribution in which the gravitational potential is determined self-consistently by the Poisson equation. Like previous authors, he was careful to note that this distribution function is not valid at large distances (outside the main body) and for high velocities (greater than the escape velocity) where the phase-space density is low. As a result, the Maxwell-Boltzmann distribution should be truncated at large energies in order to take into account the escape of high energy stars. This is how he proposed to solve the infinite mass problem.

The complete statistical mechanics problem initiated by Ogorodnikov [54, 55] was solved by Antonov [57. He neglected the escape of stars, proceeding as if the stars were confined within a sphere of radius $R$ with reflecting boundary. The physical reason advocated is that the

\footnotetext{
5 The modification of the distribution function due to the escape of stars was considered later by Woolley 29] (following Eddington 24]), Michie 30] and King 31.

6 The kinetic theory of stellar systems is reviewed in 35.

7 Ogorodnikov [54, 55] argued that the relaxation time of galaxies must be of the order of the dynamical time. As a result, in his point of view, the Maxwell-Boltzmann distribution (most probable state) cannot be established by two-body encounters (like in Ref. [5]) but by a relaxation process of a different nature that he left unspecified. This timescale problem was solved later by Lynden-Bell [56] in his theory of violent collisionless relaxation.
}

escape of stars is a slow process so that, on intermediate timescales, everything happens as if the system were confined within a box. Thus, Antonov [57] considered the problem of maximizing the Boltzmann entropy at fixed energy and particle number within a box. He showed that the Boltzmann entropy has no global maximum (there is no fully stable equilibrium state $)^{8}$ but that it may have a local maximum (corresponding to a metastable equilibrium state) with the Maxwell-Boltzmann distribution. He showed that a local entropy maximum is necessarily spherically symmetric. Furthermore, by calculating the second order variations of entropy, he showed that the density contrast of a star system with Maxwellian distribution cannot exceed 709 otherwise that Maxwellian distribution is not a local entropy maximum. In that case, the system evolves away from the Maxwell distribution and becomes non-stationary.

Lynden-Bell \& Wood [58] confirmed and extended the results of Antonov [57] by calculating the series of equilibria of self-gravitating isothermal spheres using the results known in the context of stellar structure and described in the books of Emden 21] and Chandrasekhar 22. Lynden-Bell \& Wood 58] showed that there is no equilibrium state if the energy is lower than a minimum value $E_{c}=-0.335 G M^{2} / R$ corresponding to the density contrast of 709 found by Antonov. In that case, they argued that the system takes a core-halo structure and evolves away from equilibrium: the center of the system contracts and achieves very high temperatures and densities while the halo remains cool. They explained this runaway (Antonov's instability) in terms of the negative specific heat $(C=d E / d T<0)$ of self-gravitating systems: by losing heat, the core grows hotter, contracts, and loses heat again to the profit of the halo in an unstoppable process. They called this phenomenon the "gravothermal catastrophe" (another proposed name was "thermal runaway" 59)..$^{9}$ They studied the thermodynamical stability of isothermal spheres by using the Poincaré theory of linear series of equilibria $64 .{ }^{10}$ This theory tells

\footnotetext{
8 The entropy diverges if we concentrate a part of the system and redistribute the released potential energy in another part of the system under the form of kinetic energy.

9 Their results prove that the escape of stars from a cluster is not necessary for its evolution (as was believed before their work) but rather that extended systems naturally grow a core-halo structure reminiscent of the internal constitution of a red giant star. Even when the system is confined within a box, there can be an evolution away from the Boltzmann distribution provided that the system is sufficiently centrally condensed. This can lead to the formation of a small dense nucleus which is to some extent independent of the outer parts of the system. This corroborates previous works by von Hoerner [60,62, Hénon [46, 47] and Aarseth 63. In particular, Hénon 46 47 (see also von Hoerner [62]) developed an homologous model of globular clusters leading to an infinite central density in finite time and suggested that the formation of binary stars would occur at very high densities and that this would produce a new energy source.

10 Poincaré 64 invented a powerful method for separating stable
} 
us that the instability arises at a turning point. In the microcanonical ensemble (entropy $S$ maximum at fixed energy $E$, mass $M$ and volume $V$ ), the series of equilibria becomes unstable when the density contrast is larger than 709, corresponding to the first energy peak $E_{c}$. This is the point where the specific heat vanishes $\left(C=0^{\mp}\right)$, passing from negative to positive values. In the canonical ensemble (Helmholtz free energy $F=E-T S$ minimum at fixed temperature $T$, mass $M$ and volume $V$ ), they showed that there is no equilibrium state if the temperature is lower than a minimum value $T_{c}=G M m / 2.52 k_{B} R$ corresponding to a density contrast of $32.1 .^{11}$ On the other hand, using the Poincaré criterion, they showed that the series of equilibria becomes unstable when the density contrast is larger than 32.1 , corresponding to the first temperature peak $T_{c}$. This is the point where the specific heat becomes infinite $(C= \pm \infty)$, passing from positive to negative values. They also considered other ensembles making the connection with the earlier works of Ebert [67, 68, Bonnor 69] and McCrea 70] (Gibbs free energy $G=E-T S+P V$ minimum at fixed temperature $T$, mass $M$ and pressure $P$ ) and with the SchönbergChandrasekhar 71] limit (corresponding approximately to the canonical ensemble). Therefore, the onset of instability is different depending whether the energy or the temperature are held fixed. This corresponds to what is now called ensembles inequivalence for systems with longrange interactions in statistical mechanics. Lynden-Bell and Wood [58 mentioned this inequivalence (see their footnote p. 509) and connected it with the existence of negative specific heats that are allowed in the microcanonical ensemble but not in the canonical ensemble (see Appendix B). Finally, they related the absence of global entropy maximum with the formation of a subset of particles very closely bound together by gravity, like a binary star. However, they mentioned that the formation of binary stars is a rare event so we can consider "frozen equilibria" (corresponding to metastable states) in which the number of binaries is unchanged in the time available.

Thirring 72 studied the statistical mechanics of selfgravitating systems without being aware of previous works on the subject (he mentioned the work of LyndenBell and Wood [58] as a Note added in proof). He emphasized the fact that self-gravitating systems may have negative specific heats in the microcanonical ensemble while the specific heat is necessarily positive in the canonical ensemble (see Appendix B). He concluded therefore that the ensembles are not equivalent. He showed that the

from unstable equilibria. It is based on series of equilibrium configurations. The method was used by Poincaré and many others (see for instance Jeans [9], Lyttleton [65] and Ledoux [66]) to find stable equilibria of rotating liquid masses and rotating systems of rigid bodies.

11 This result was actually discovered by Emden 21] (see Chap. $\mathrm{XI})$. microcanonical entropy, defined as the logarithm of the density of states, diverges if one particle is sent to infinity or if two of them are approached at infinitely close distances. It is therefore necessary to introduce a box and a small distance repulsion. Using a saddle point approximation, he derived the mean field Boltzmann distribution in which the gravitational potential is produced by the system as a whole.

Horwitz and Katz [73, 74] (independently from Thirring [72]) developed a field theory based on path integrals to study the equilibrium statistical mechanics of stellar systems. Their approach uses a rigorous microcanonical formulation which produces an exact functional integral expression for the density of states and the entropy. ${ }^{12}$ This integral is evaluated by steepest-descent methods, the saddle-point value giving the mean field entropy (Boltzmann entropy) and the analysis of quadratic fluctuations yielding conditions of stability. This provides an approximation which is mean field plus fluctuations. Horwitz and Katz [73, 74, determined sequences of equilibrium states which are presumed to simulate slowly evolving, near-equilibrium, configurations of real star clusters. They considered different ensembles (microcanonical, canonical and grand canonical) and obtained stability criteria in the form of eigenvalue equations involving the Schrödinger operator introduced by Lynden-Bell and Sanitt 75. They also showed that nonradial perturbations are stable in all ensembles. Using the Poincaré 64 criterion, they showed that instability occurs at the turning point of an appropriate thermodynamic potential. Therefore, the onset of instabilities for spherical perturbations can be associated with the sign change of standard thermodynamic functions such as the heat capacity. ${ }^{13}$ Finally, they noted that a system which

12 True statistical equilibrium for particles interacting gravitationally with an $r^{-1}$ law is impossible because the statistical integral diverges both when a particle moves out to infinity, as well as when two particles approach one another indefinitely. The ultimate configuration, reached for $t \rightarrow+\infty$, consists in a hard binary plus $N-2$ high velocity stars. However, the evaporation of particles proceeds slowly and the formation of binaries is a rare event. We can therefore consider near equilibrium states of the system with given energy and particle number. Horwitz and Katz 73, 74] confined the stars to a finite volume (box) in order to eliminate evaporation and introduced an appropriate short distance cut-off in the interparticle interaction so as to eliminate tightly bound pairs. This is necessary to make their integrals convergent. However, at the level of the mean field approximation, they showed that the short distance cutoff can finally be taken to zero.

13 Generally, constraints act to stabilize the system. The microcanonical which constrains both the energy and the mass is the most stable (with a critical density constrast of 709), while the grand canonical ensemble which allows both the energy and the mass to fluctuate (at fixed temperature and chemical potential) is the least stable (with a critical density constrast of 1.58). The canonical ensemble which allows the energy to fluctuate (at fixed temperature) but constrains the mass lies between the two (with a critical density constrast of 32.1 ). 
is thermodynamically stable is also dynamically stable.

Katz [76, 77] generalized the turning point criterion of Poincaré when there are more than one turning point and applied it to thermodynamical problems thereby recovering the results of Lynden-Bell and Wood [58] and Horwitz and Katz 74]. The Poincaré criterion allows one to determine the thermodynamical stability of the system from topological properties of continuous series of equilibria (provided stability conditions are known for one configuration) without having to solve an eigenvalue equation. In this connection, Katz [76] plotted for the first time (by hand) the caloric curve $\beta(E)$ giving the inverse temperature as a function of the energy ( $\beta$ is the variable conjugate to $E$ with respect to $S$ ). This curve has a striking spiralling (snail-like) shape. The series of equilibria is parametrized in terms of the density contrast which grows as one spirals inwards. The series of equilibria becomes unstable at the first energy minimum in the microcanonical ensemble and at the first temperature minimum in the canonical ensemble as previously showed by Lynden-Bell and Wood [58. However, Katz [76] showed that more and more modes of stability are lost (more and more eigenvalues become negative) as one rotates clockwise along the spiralling series of equilibria.

Lecar and Katz [78] introduced a new ensemble, called the grand microcanonical ensemble which constrains the energy but allows the mass to fluctuate (at fixed chemical potential). Using the turning point method they found that the series of equilibria is successively stable, unstable, stable again, and finally unstable.

Padmanabhan [79] wrote the first review on the statistical mechanics of self-gravitating systems. In his review, and in Ref. 80, he presented a simplified derivation of the Antonov instability and gravothermal catastrophe by explicitly solving the zero eigenvalue equation associated with the second variations of the entropy. ${ }^{14}$ This solution allowed him to study the nature of the mode that triggers the instability in the microcanonical ensemble. He proposed a very elegant graphical method to obtain the point of marginal stability and the form of the perturbation at the critical point. He found that that this mode has a "core-halo" structure (the density perturbation $\delta \rho$ has two nodes).

Chavanis [89, 90] applied the approach of Padmanabhan [79, 80] to the canonical, grand-canonical and grand-microcanonical ensembles, thereby recovering and extending the results previously obtained by Lynden-Bell and Wood [58, Horwitz and Katz [74, Katz 76, 77], and Lecar and Katz [78. He also considered the isobaric ensembles previously studied by Ebert 67, 68, Bonnor 69. and McCrea [70. In the canonical ensemble, he showed that thermodynamical stability coincides with dynam-

\footnotetext{
14 See also the previous works of Ebert [68, Yabushita [81, 82], Taff and van Horn 83, 84, Nakada 85, Hachisu and Sugimoto 86, Hachisu et al. 87. and Inagaki 88 .
}

ical stability (with respect to the Euler-Poisson equations) and that the mode of marginal instability has a "core" structure (the density perturbation $\delta \rho$ has just one node) contrary to the "core-halo" structure found by Padmanabhan [79, 80, in the microcanonical ensemble. As a consequence, in the microcanonical ensemble the gravothermal catastrophe [58] below $E_{c}$ ultimately leads to a binary star surrounded by a hot halo (this structure has an infinite entropy $S \rightarrow+\infty$ at fixed energy) while in the canonical ensemble the isothermal collapse 89. below $T_{c}$ ultimately leads to a Dirac peak containing all the particles (this structure has an infinite free energy $F \rightarrow-\infty)$. This is another manifestation of ensembles inequivalence. For isolated self-gravitating systems described by an $N$-body Hamiltonian system, like galaxies and globular clusters, only the microcanonical ensemble is relevant. The other ensembles have no physical meaning. ${ }^{15}$ However, the canonical ensemble is rigorously justifed for the model of self-gravitating Brownian particles introduced and studied by Chavanis and Sire [91 97].

Katz \& Okamoto 98 (see also the review of Katz 99]) studied temperature fluctuations in self-gravitating isothermal spheres and showed that the onset of gravitational instability (gravothermal catastrophe) is advanced because of finite $N$ effects. The critical density contrast taking into account the finite number of particles is $\mathcal{R}_{c}=709 \times \exp \left(-3.30 N^{-1 / 3}\right)$. This may explain why observations reveal that a greater number of globular clusters than is normally believed may already be in an advanced stage of core collapse.

Chavanis 100] (see also the review 101]) argued that the lifetime of a self-gravitating system trapped in a metastable state (local entropy maximum) scales as $e^{N \Delta s}$, where $\Delta s$ is the barrier of entropy per particle. ${ }^{16}$ For $N \gg 1$, the lifetime of a metastable state is exponentially large making it of extreme physical relevance. ${ }^{17}$ As a result, globular clusters that lie on the series of equilibria before the point of instability can be considered as

15 For systems with long-range interactions one cannot deduce the canonical ensemble (and the other ensembles) from the microcanonical ensemble by considering a subsystem of a large ensemble because the energy is nonadditive (the sum of energies of all the small sub-systems is not equal to the total energy of the system). Nevertheless, a mathematical interest of considering less constrained ensembles is that they are simpler to study and that they provide sufficient conditions of thermodynamical stability.

16 In the canonical ensemble the lifetime of a metastable state is given by the Kramers formula $e^{N \Delta f / k_{B} T}$, where $\Delta f$ is the barrier of free energy per particle.

17 For $t \rightarrow+\infty$, a self-gravitating system trapped in a metastable state is expected to ultimately collapse and form binaries since there is no global entropy maximum. However, the system may find itself "blocked" in a local entropy maximum for a very long time, of the order of $e^{N} t_{D}$, much larger than the age of the Universe. Only a large random fluctuation can drive the system out of this local maximum of entropy. This is a rare event. This makes local entropy maxima extremely important on the time scales relevant in astrophysics. 
long-lived metastable states. Chavanis [100] calculated the barrier of entropy close to the critical point $E_{c}$ and recovered the threshold of gravitational collapse due to finite $N$ effects obtained by Katz \& Okamoto [98].

de Vega and Sanchez [102, 103] (see also [104-106]) studied the statistical mechanics of the self-gravitating gas using field theory, thereby confirming and complementing the former works of Horwitz and Katz [73, 74]. The statistical mechanics approach (writing the density of states or the partition function as path integrals and evaluating them by making a saddle point approximation) is more rigorous than the thermodynamical method (maximizing or minimizing a relevant thermodynamic potential to obtain the most probable state) but it is considerably more formal and complicated. It can be shown that the statistical mechanics approach gives exactly the same results as the thermodynamic approach in a proper thermodynamic limit where the number of particles $N \rightarrow+\infty$ keeping $\Lambda=-E R / G M^{2}$ and $\eta=\beta G M m / R$ fixed [101]. We refer to [107 110] for rigorous mathematical results on this subject.

The works that we have reviewed so far consider boxconfined isothermal stellar systems. However, real stellar systems like globular clusters, are not in boxes and particles with high enough energy can escape from the system. As a result, the distribution function is not exactly given by the Boltzmann distribution (especially for high energies) and truncated models have been introduced, notably the Woolley model [29] and the Michie-King model [30, 44] (see footnote 5). The thermodynamical stability of these models has been studied by analogy with the thermodynamics of box-confined systems [58, 111 114. The caloric curves of the Woolley and Michie-King models have the form of spirals. The stability limits can be determined from the Poincaré criterion by identifying the turning point of energy in the series of equilibria. As first suggested by Lynden-Bell and Wood [58] and Horwitz and Katz 73], the sequences of equilibrium states are presumed to simulate slowly evolving near-equilibrium configurations of real stellar systems.

The physical picture that emerges from these studies is the following. Because of stellar encounters and evaporation, the globular clusters slowly evolve along a series of equilibria corresponding to the King model. The evolution is such that the central density increases and the energy decreases. ${ }^{18}$ In the region of positive specific heat, the temperature decreases while it increases in the

\footnotetext{
18 This can be understood as follows. Under the effect of close encounters, stars leave the system with an energy positive or close to zero. Therefore, the energy of the cluster decreases or remains approximately constant. Since the number of stars in the cluster decreases, the cluster contracts (according to the virial theorem) and becomes more and more concentrated. Therefore, the central density increases with time. Another argument, related to the $H$ theorem, explaining why the central density naturally increases with time is developed in 1 .
}

region of negative specific heat. The evolution continues until the point of instability, corresponding to the turning point of energy (energy minimum). This is when the specific heat vanishes. At that point, the system undergoes the Antonov instability [57, also known as the gravothermal catastrophe [58, and collapses. This instability can be followed by using dynamical models based either on moment equations derived from the Fokker-Planck equation 115, 116, Monte Carlo models [117, $N$-body simulations [118, heuristic fluid equations [87, 119], or kinetic equations such as the orbit-averaged-Fokker-Planck equation [120. During the collapse the system takes a core-halo structure (reminiscent of a red giant) in which the cluster develops a dense and "hot" core and a diffuse envelope. The dynamical evolution of the system is due to the gradient of temperature (velocity dispersion) between the core and the halo and the fact that the core has a negative specific heat (see Appendix B). The core loses heat to the profit of the halo, becomes hotter, and contracts. If the temperature increases more rapidly in the core than in the halo there is no possible equilibrium and we get a thermal runaway: this is the gravothermal catastrophe. As a result, the core collapses and reaches higher and higher densities and higher and higher temperatures while the halo is not sensibly affected by the collapse of the core and maintains its initial structure (it remains cool). The collapse of the core is self-similar and leads to a finite time singularity: the central density and the temperature become infinite in a finite time while the core radius shrinks to nothing [119, 120. This is called core collapse. The mass contained in the core tends to zero at the collapse time. In the case of globular clusters, the evolution continues in a self-similar postcollapse regime [121. with the formation of a binary star containing a significant fraction of the cluster energy (as previously found by Aarseth [118 in his $N$-body simulations). The energy released by the binary can stop the collapse and induce a reexpansion of the system. Then, a series of gravothermal oscillations is expected to follow [122, $123 .{ }^{19}$

It has to be stressed that the gravothermal catastrophe is a thermodynamical instability, not a dynamical instability. Indeed, it has been shown that all isotropic stellar systems with a distribution function of the form $f(\epsilon)$ with $f^{\prime}(\epsilon)<0$, including the truncated Maxwell-Boltzmann (isothermal) distribution, are dynamically stable with respect to the collisionless Vlasov-Poisson equations [124-

19 Analogously, the isothermal collapse of self-gravitating Brownian particles in the canonical ensemble when $T<T_{c}$ can be followed by solving the Smoluchowski-Poisson equations [91. The collapse of the system is self-similar and leads to a finite time singularity: the central density becomes infinite in a finite time while the core radius and the core mass vanish [92. The evolution continues in a self-similar postcollapse regime with the formation of a Dirac peak progressively accreting all the mass [93. 
129. In particular, all the isothermal configurations on the series of equilibria are dynamically stable, even those deep into the spiral that are thermodynamically unstable. Therefore, dynamical and thermodynamical stability do not coincide in Newtonian gravity (thermodynamical stability implies dynamical stability but the converse is wrong [130, 131]). This implies that the gravothermal catastrophe is a very long (secular) process, occurring on a collisional relaxation timescale of the order of the age of the Universe, not on a fast dynamical timescale.

The statistical mechanics of relativistic star clusters ${ }^{20}$ started with the seminal work of Zel'dovich and Podurets 132. They took into account collisions and evaporation and studied star clusters described by the truncated Maxwell-Boltzmann distribution in full general relativity. $^{21}$ This distribution has a finite mass so there is no need to introduce an artificial box to confine the system. They considered a series of quasi-equilibrium states and plotted the temperature $T_{\infty}$ measured by an infinitely-remote observer as a function of the central density $\rho_{0}$. They found that the function $T_{\infty}\left(\rho_{0}\right)$ rises up to a maximum temperature $k_{B}\left(T_{\infty}\right)_{\max } / m c^{2}=0.273$ then undergoes damped oscillations. ${ }^{22}$ As a result, equilibrium states can exist only below a maximum temperature $\left(T_{\infty}\right)_{\max }$. They heuristically argued that the series of equilibria becomes unstable after the first turning point of temperature and that a new mode of instability appears at each turning point of temperature. These considerations led them to the following scenario. Because of collisions and evaporation, a star cluster slowly (secularly) evolves through a sequence of quasiequilibrium states. During this evolution both the temperature and the central density increase. At a certain point the system has high temperatures, large velocities $v \sim c$, and is therefore general relativistic even if during the initial stage it was Newtonian $(v \ll c)$. When the temperature reaches $\left(T_{\infty}\right)_{\max }$ the system becomes unstable and undergoes a catastrophic gravitational collapse. This is what they called an "avalanche-type catastrophic contraction of the system." The mechanism of the collapse proposed by Zel'dovich and Podurets 132 is the following. The orbits of highly relativistic particles become unstable and the corresponding particles start falling in spirals towards the center. The collapse of the orbits of some particles leads to an increase of the field acting on the other particles, whose orbits collapse in turn etc.

20 They may be clusters of stars like white dwarfs, neutron stars or stellar mass black holes in galactic nuclei.

21 Truncated isothermal distributions of relativistic star clusters have been studied independently by Fackerell 133 .

22 They mentioned that the damped oscillations of $T\left(\rho_{0}\right)$ are similar to the damped oscillations of $M\left(\rho_{0}\right)$ for neutron stars discovered by Dmitriev and Kholin [134. This is because, in the ultrarelativistic limit where the density is large, the equation of state of a classical isothermal gas takes the form $P=\epsilon / 3$, where $\epsilon$ is the energy density, like the ultrarelativistic equation of state of a Fermi gas at $T=0$.
This catastrophic collapse occurs rapidly, on a dynamical timescale. A large fraction of the system (the main mass) rapidly contracts to its gravitational radius and forms what is now called a black hole. ${ }^{23}$ However, only the core of the system collapses. There remains a cloud surrounding the main mass. The particles in the cloud, following the laws of slow evolution, gradually fall into the collapsed mass.

Ipser [140] studied the dynamical stability with respect to the Vlasov-Einstein equations of isothermal relativistic star clusters with heavily truncated Maxwell-Boltzmann velocity distribution ${ }^{24}$ by using the equation of pulsations derived by Ipser and Thorne [141, 142. He showed that the clusters are unstable against gravitational collapse if the redshift $z_{0}$ of a photon emitted from its center and received at infinity is larger than 0.516 . The clusters are likely to be stable if $z_{0} \leq 0.516$. Ipser 140 also plotted the fractional binding energy $E / N m c^{2}=(M-N m) / N m$ as a function of the central redshift $z_{0}$ and showed that it reaches a minimum before undergoing damped oscillations. Interestingly, the point of onset of gravitational collapse $\left(z_{c}=0.516\right)$ appears to coincide with the first turning point of fractional binding energy (minimum). At that point $z_{c}=0.516,[(M-N m) / N m]_{c}=0.0357$, $\left(R c^{2} / 2 G N m\right)_{c}=4.42$ and $\left(k_{B} T_{\infty} / m c^{2}\right)_{\mathrm{c}}=0.23$. This is different from the turning point of temperature reported by Zel'dovich and Podurets [132] corresponding to $z_{0}=1.08,(M-N m) / N m=0.0133, R c^{2} / 2 G N m=$ 3.92 , and $k_{B}\left(T_{\infty}\right)_{\max } / m c^{2}=0.27$. In particular, the gravitational instability occurs sooner than predicted by Zel'dovich and Podurets [132.

Based on these results, Fackerell et al. [151] developed a scenario for the evolution of spherical relativistic star clusters improving the original picture of Zel'dovich and Podurets 132. A protocluster is expected to relax towards a relativistic isothermal distribution, ${ }^{25}$ after which it might evolve quasistatically along a series of equilibria by means of stellar collisions and by the evaporation of stars. Both collisions and evaporation should drive

23 The name "black hole" was popularized by Wheeler 135, 136 but it appeared earlier 137 138, being probably introduced by Dicke in analogy with the Black Hole prison of Calcutta (see Ref. [139]).

24 Following Zel'dovich and Podurets [132], Ipser [140] assumed a certain relation between the energy cutoff and the temperature. This relatively ad hoc choice was later criticized. This led to several generalizations of the problem by Katz et al. [143, Suffern and Fackerell 144, Fackerell and Suffern [145], Merafina and Ruffini 146 148, and Bisnovatyi-Kogan et al. 149, 150, that we do not review here.

25 They noted that if Newtonian stellar systems evolve only through evaporation they will never reach, on a relevant timescale, relativistic densities (as assumed by Zel'dovich and Podurets [132]). However, they added that recent studies by Antonov [57] and Lynden-Bell and Wood [58] indicate there is a rapid evolution by a "thermal runaway" in which the cluster develops a dense and hot core and a diffuse envelope on a timescale that could be less than $10^{10}$ years for the nuclei of some galaxies. 
the cluster towards states of tighter and tighter binding. Indeed, when a star is ejected from a cluster, it carries away nonzero kinetic energy as measured by an observer at infinity, and thereby decreases the fractional binding energy of the cluster; when two stars collide and stick they increase the cluster's rest mass and hence decrease its binding energy. When the cluster reaches the point of minimum fractional binding energy it can no longer evolve quasistatically and a catastrophic relativistic gravitational collapse ensues: the stars spiral inward through the gravitational radius of the cluster towards its center leaving behind a "black hole" in space with perhaps some stars orbiting it. ${ }^{26}$ They speculated that violent events in the nuclei of galaxies and in quasars might be associated with the onset of such a collapse or with encounters between an already collapsed cluster (black hole) and surrounding stars.

The thermodynamics of relativistic truncated isothermal star clusters (relativistic Woolley model) and relativistic isothermal clusters in a box was also studied by Katz and Horwitz [152] (see also [143, 153]) who extended their path integral approach and steepest descent techniques 73 , 74, 111 to general relativity. They showed that extrema (saddle points) of the action give the Einstein equations (including the condition of mechanical equilibrium) and the Tolman-Klein relations (uniformity of global temperature and global chemical potential). For box-confined systems, the mean field action is equal to the Boltzmann entropy and the mean field equilibrium distribution is the Maxwell-Boltzmann distribution. They derived a criterion of thermodynamical stability in terms of a relativistic "Schrödinger operator" extending the one introduced by Lynden-Bell and Sanitt [75] in Newtonian gravity. ${ }^{27}$ Using the Poincaré turning point criterion, Horwitz and Katz 154] showed that the change of thermodynamical stability in the microcanonical ensemble occurs at the turning point (minimum) of binding energy with respect to the central redshift along the series of equilibria at fixed $N$. This is when the heat capacity becomes equal to zero. Therefore, truncated isothermal spheres are thermodynamically stable before the turning point of energy $\left(z_{0}<0.516\right)$ and thermodynamically unstable after the turning point of energy $\left(z_{0}>0.516\right)$. This coincides with the dynamical stability results of Ipser [140].

Ipser [155] (see also [130, 156] in Newtonian gravity) considered the maximization of an arbitrary "entropic" functional $S$ at fixed mass-energy $M c^{2}$ and particle number $N$. This variational principle describes a large class of relativistic clusters that are not necessarily isothermal. He showed that the extermization problem determines an

${ }^{26}$ For a fluid sphere (star) the collapse is a radial infall of all the fluid. For a star cluster it is an inward spiralling of all the stars.

27 Their paper [152] (and [73] in Newtonian gravity) contains mistakes due to the inequivalence of statistical ensembles that were corrected in [154] (and 74, 111] in Newtonian gravity). isotropic stationary solution of the Vlasov-Einstein equations (for maxwellian clusters the Tolman-Klein relations are automatically satisfied in this formulation). He also showed that a distribution function that is a maximum of entropy $S$ at fixed mass-energy $M c^{2}$ and particle number $N$ is necessarily dynamically stable with respect to the Vlasov-Einstein equations. Therefore thermodynamical stability (in a general sense) implies dynamical stability. Using the Poincaré criterion, he obtained a binding energy stability theorem: "an isotropic relativistic star cluster is dynamically stable at least up to the turning point of fractional binding energy." This theorem is valid both for Newtonian clusters (as previously showed in [130]) and general relativistic clusters. Considering isothermal clusters as a particular case, it implies that the configurations with $z_{0}<0.516$ are both thermodynamically and dynamically stable. On the other hand, configurations with $z_{0}>0.516$ turn out to be dynamically unstable (as shown numerically in [140]) in addition of being thermodynamically unstable. Ipser [155] therefore concluded that, in general relativity, thermodynamical stability coincides with dynamical stability. He conjectured that this result remains valid for an arbitrary "entropic" functional so that, in general relativity, all isotropic star clusters become dynamically unstable after the turning point of binding energy. This is in sharp contrast with the Newtonian case where it has been shown 124 129. that all isotropic models are dynamically stable with respect to the Vlasov-Poisson equations, even those that lie after the turning point of energy.

These theoretical results have been confirmed by Shapiro and Teukolsky 157 161 who numerically solved the relativistic Vlasov-Einstein equations governing the dynamical evolution of a collisionless spherical gas of particles in general relativity. They followed the series of equilibria of truncated isothermal distributions (assumed to result from the gravothermal catastrophe of initially Newtonian clusters) and showed from direct numerical simulations that above a critical redshift $z_{c} \sim 0.516$, corresponding to the turning point of fractional binding energy, the relativistic star cluster becomes dynamically unstable and undergoes a catastrophic collapse to a supermassive black hole on a dynamical time scale.

Sorkin et al. 162 and more recently Chavanis 163 . studied the thermodynamics of a self-gravitating blackbody radiation confined within a cavity in general relativity. Black-body radiation is equivalent to an ultrarelativistic gas of massless bosons (photons) with a linear equation of state $P=\epsilon / 3$, where $\epsilon$ denotes the energy density. ${ }^{28}$ The equilibrium state of the selfgravitating black-body radiation is obtained by maximizing the entropy $S$ (proportional to the particle number

28 This equation of state also corresponds to the ultrarelativistic limit of an ideal gas of any kind of massive particles, classical, fermionic or bosonic. 
$N)$ at fixed mass-energy $M c^{2}$. This leads to the TolmanOppenheimer-Volkoff [164, 165] equations expressing the condition of hydrostatic equilibrium and to the Tolman 164 relation. The caloric curve $T_{\infty}(\mathcal{E})$, where $T_{\infty}$ denotes the Tolman temperature and $\mathcal{E}=M c^{2}$ the massenergy, forms a spiral so that no equilibrium state exists above a maximum energy $\mathcal{E}_{\max }=0.24632 R c^{4} / G$ for an isolated system or above a maximum temperature $k_{B}\left(T_{\infty}\right)_{\max }=0.445\left(\hbar^{3} c^{7} / G R^{2}\right)^{1 / 4}$ for a system in contact with a heat bath (see Fig. 15 of [163). Using different methods, Sorkin et al. 162 and Chavanis 163 showed that thermodynamical stability coincides with dynamical stability with respect to the Euler-Einstein equations and that the series of equilibria becomes unstable after the first turning point of energy in agreement with the Poincare criterion. This is when the specific heat $C=d \mathcal{E} / d T_{\infty}$ vanishes, passing from negative to positive values. This corresponds to an energy density contrast $\mathcal{R}_{\mathrm{MCE}}=22.4$ [163]. The system becomes unstable when it is "too hot" because energy is mass so that it gravitates. This is what Tolman 164 called the "weight of heat". The mode of marginal instability at $\mathcal{E}_{\max }$ has a "core" structure (the energy density perturbation $\delta \epsilon$ has just one node) [163, 166]. Therefore, gravitational collapse is expected to lead to the formation of a black hole.

The statistical mechanics of general relativistic classical self-gravitating systems confined within a box was reconsidered recently by Roupas [167] and, independently, by us (our study was made during the $\mathrm{PhD}$ thesis of $\mathrm{G}$. Alberti from 2014 to 2017). In this paper, we report our results which confirm and complete the results obtained by Roupas [167]. A comparison between the two studies is made in the conclusion. The paper is organized as follows. In Sec. II we recall the main equations governing the structure of a general relativistic classical gas at statistical equilibrium. In Sec. III we recall the caloric curve of a nonrelativistic classical self-gravitating gas (cold spiral) and the caloric curve of the self-gravitating blackbody radiation (hot spiral). In Sec. IV] we discuss a typical example where the caloric curve of a general relativistic classical gas presents a double spiral connecting the cold and hot spirals found previously. In Sec. $\mathrm{V}$ we treat the general case and show how the caloric curve changes as we increase the number of particles. In Sec. VI we consider the limit $N \rightarrow 0$. We introduce two types of normalization of energy and temperature, appropriate to the nonrelativistic and ultrarelativistic limits, in order to obtain asymptotic caloric curves describing the cold and hot spirals respectively. We discuss the analogies and the differences between the caloric curve of an ultrarelativistic classical gas and the caloric curve of the self-gravitating black-body radiation. In Sec. VII we study the evolution of the critical points of the caloric curves as a function of $N$ and obtain explicit asymptotic results. The extension of our results to self-gravitating fermions in general relativity is made in our companion paper 2] (see also [168, 169]). A summary of our main results is presented in 170 .

\section{BASIC EQUATIONS OF A GENERAL RELATIVISTIC CLASSICAL GAS}

In this section, we recall the basic equations describing the structure of a general relativistic classical gas at statistical equilibrium (see 1, 3, 167] for their derivation). In order to make the connection with our companion paper 2], we assume that this classical gas corresponds to the nondegenerate limit of a gas of fermions. At statistical equilibrium it is described by the Maxwell-Juttner distribution

$$
f(\mathbf{r}, \mathbf{p})=\frac{g}{h^{3}} e^{\alpha} e^{-E(p) / k_{B} T(r)},
$$

where $E(p)=\sqrt{p^{2} c^{2}+m^{2} c^{4}}$ is the energy of a particle. The temperature and the chemical potential are spacedependent. They are given by the Tolman-Klein relations $T(r)=T_{\infty} e^{-\nu(r) / 2}$ and $\mu(r)=\mu_{\infty} e^{-\nu(r) / 2}$, where $T_{\infty}$ and $\mu_{\infty}$ are the temperature and chemical potential measured by an observer at infinity and $\nu(r)$ is the metric coefficient. $T_{\infty}$ will be called the Tolman (global) temperature [164] and $\mu_{\infty}$ will be called the Klein (global) chemical potential [171]. Since the local temperature $T(r)$ and the local chemical potential $\mu(r)$ are red-shifted in the same manner, their ratio

$$
\alpha=\frac{\mu(r)}{k_{B} T(r)}=\frac{\mu_{\infty}}{k_{B} T_{\infty}}
$$

is uniform throughout the system.

The number density $n(r)=\int f d \mathbf{p}$, the energy density $\epsilon(r)=\int f E(p) d \mathbf{p}$ and the pressure $P(r)=$ $(1 / 3) \int f p E^{\prime}(p) d \mathbf{p}$ are related to the local temperature $T(r)$ and to the local chemical potential $\mu(r)$ by

$$
\begin{gathered}
n(r)=\frac{4 \pi g m^{3} c^{3}}{h^{3}} e^{\alpha} \frac{1}{b(r)} K_{2}(b(r)) \\
\epsilon(r)=\frac{4 \pi g m^{4} c^{5}}{h^{3}} e^{\alpha} \frac{1}{b(r)} K_{2}(b(r))\left[\frac{K_{1}(b(r))}{K_{2}(b(r))}+\frac{3}{b(r)}\right] \\
P(r)=n(r) \frac{m c^{2}}{b(r)}
\end{gathered}
$$

where $K_{n}(z)$ are the modified Bessel functions. In the foregoing equations, we have introduced the normalized local inverse temperature

$$
b(r)=\frac{m c^{2}}{k_{B} T(r)}
$$

and the parameter $\alpha$ defined by Eq. (2). Eqs. (3)(5) define the equation of state of a relativistic classical gas in parametric form. By construction, $b(r) \geq 0$ and $-\infty<\alpha<+\infty$.

The Tolman-Oppenheimer-Volkoff (TOV) equations [164, 165], which correspond to the equation of hydrostatic equilibrium in general relativity, can be written as

$$
\frac{d M}{d r}=\frac{\epsilon(r)}{c^{2}} 4 \pi r^{2},
$$




$$
\frac{1}{b(r)} \frac{d b}{d r}=\frac{1}{c^{2}} \frac{\frac{G M(r)}{r^{2}}+\frac{4 \pi G}{c^{2}} P(r) r}{1-\frac{2 G M(r)}{r c^{2}}},
$$

where $M(r)$ is the mass-energy contained within the sphere of radius $r$. They have to be solved with the boundary conditions

$$
M(0)=0, \quad b(0)=b_{0} \geq 0 .
$$

We assume that the system is confined within a spherical box of radius $R$ (as recalled in the Introduction, a box is necessary to prevent the evaporation of the gas and have a well-defined statistical equilibrium state). The massenergy of the gas and the particle number are then given by

$$
\begin{gathered}
M=M(R)=\frac{1}{c^{2}} \int_{0}^{R} \epsilon(r) 4 \pi r^{2} d r \\
N=\int_{0}^{R} n(r)\left[1-\frac{2 G M(r)}{r c^{2}}\right]^{-1 / 2} 4 \pi r^{2} d r .
\end{gathered}
$$

The Tolman temperature can be obtained from the relation

$$
T_{\infty}=T(R) \sqrt{1-\frac{2 G M}{R c^{2}}},
$$

where $T(R)$ is the temperature of the system on the box. Finally, the entropy is given by

$S=\int_{0}^{R} \frac{\epsilon(r)}{T(r)}\left[1-\frac{2 G M(r)}{r c^{2}}\right]^{-1 / 2} 4 \pi r^{2} d r+k_{B} N-\alpha k_{B} N$,

and the free energy by

$$
F=E-T_{\infty} S
$$

where

$$
E=(M-N m) c^{2}
$$

is the binding energy. ${ }^{29}$

In the following, in order to be consistent with our companion paper [2], we shall express the results in terms of the relativistic gravitational potential $\Phi(r)$ defined by ${ }^{30}$

$$
\frac{k_{B} T(r)}{m c^{2}}=\frac{1}{b(r)}=\frac{1}{|\alpha|} \sqrt{1+\frac{\Phi(r)}{c^{2}}}
$$

\footnotetext{
29 The binding energy is usually defined with the opposite sign, i.e., $E_{b}=(N m-M) c^{2}$. We shall, however, call $E$ the binding energy or, simply, the energy. In the nonrelativistic limit $c \rightarrow+\infty$, it reduces to the Newtonian energy $E=E_{\text {kin }}+W$ (kinetic + potential).

30 The gravitational potential is denoted $\varphi(r)$ in [1.
}

instead of the temperature $T(r)$. By construction, $\Phi(r) \geq-c^{2}$. The method used to obtain the caloric curve $T_{\infty}(E)$ is explained in Appendix C In order to make contact with the nonrelativistic results, we shall use the dimensionless energy $\Lambda$ and the dimensionless inverse Tolman temperature $\eta$ defined by

$$
\Lambda=-\frac{E R}{G N^{2} m^{2}} \quad \eta=\frac{\beta_{\infty} G N m^{2}}{R},
$$

In the microcanonical ensemble, a stable equilibrium state corresponds to a maximum of entropy $S$ at fixed energy $E$ and particle number $N$. In the canonical ensemble, a stable equilibrium state corresponds to a minimum of free energy $F$ at fixed particle number $N$. The stability of the system can be settled by plotting the caloric curve (or series of equilibria) $\eta(\Lambda)$ and using the Poincaré 64 turning point criterion.

As shown in 1, 167, the caloric curve of the general relativistic classical gas depends on a single control parameter

$$
\nu=\frac{G N m}{R c^{2}},
$$

called the compactness parameter. It can be interpreted as the ratio $\nu=R_{S}^{*} / R$ between an effective Schwarzschild radius $R_{S}^{*}=G N m / c^{2}$, defined in terms of the rest mass $N m$, and the box radius $R$. Alternatively, $\nu=N m / M_{S}^{*}$ where $M_{S}^{*}=R c^{2} / G$ is an effective Schwarzschild mass. Using the normalized variables introduced in Appendix B of [2] we can take $\hbar=c=G=m=g / 2=R=1$ without restriction of generality. In that case, we get

$$
\Lambda=-\frac{E}{N^{2}} \quad \eta=\beta_{\infty} N \quad \nu=N .
$$

\section{PARTICULAR LIMITS}

In this section, we recall the caloric curve of a nonrelativistic classical self-gravitating gas and the caloric curve of the self-gravitating black-body radiation. They will help us interpreting the nonrelativistic and ultrarelativistic limits of the general relativistic classical gas.

\section{A. Nonrelativistic classical self-gravitating gas}

The thermodynamics of a nonrelativistic classical selfgravitating gas confined within a box has been studied in detail in [57, 58, 72,74, 76, 80, 89, 90, 98, 100, 102 104. It is described by a linear equation of state $P(r)=$ $\rho(r) k_{B} T / m$ where $T$ is uniform throughout the system. The natural dimensionless energy and inverse temperature are

$$
\Lambda=-\frac{E R}{G M^{2}} \quad \eta=\frac{\beta G M m}{R},
$$

where we recall that $M=N m$ for nonrelativistic systems. The caloric curve (or series of equilibria) $\eta(\Lambda)$ has 
the form of a spiral (see Fig. 1) parametrized by the density contrast $\mathcal{R}=\rho(0) / \rho(R)$. It was first plotted by Katz [76. This spiral is implicit in the papers of Antonov [57. and Lynden-Bell and Wood [58. It also appears (plotted in terms of other variables) in earlier works on isothermal stars [21, 22, 67, 71].

In the microcanonical ensemble there is no equilibrium state below a minimum energy $E_{c}$ given by [58]

$$
\Lambda_{\mathrm{c}}=-\frac{E_{c} R}{G M^{2}}=0.335
$$

Using the Poincaré criterion 64, one can show that the series of equilibria becomes and remains unstable after the first turning point of energy $\Lambda_{c}$ (a new mode of stability is lost at each turning point of energy). This is when the specific heat $C=d E / d T$ vanishes. This corresponds to a density contrast $\mathcal{R}_{\mathrm{MCE}}=709$ [57. At that point, the system undergoes a gravothermal catastrophe [58. In the case of globular clusters, this gravitational collapse ultimately leads to the formation of a binary star surrounded by a hot halo [119 121]. This structure has an infinite entropy $S \rightarrow+\infty$ at fixed energy (see Appendix A of 92]).

In the canonical ensemble there is no equilibrium state below a minimum temperature $T_{c}$ given by [21]

$$
\eta_{c} \equiv \frac{\beta_{c} G M m}{R}=2.52 .
$$

Using the Poincaré criterion 64, one can show that the series of equilibria becomes and remains unstable after the first turning point of temperature $\eta_{c}$ (a new mode of stability is lost at each turning point of temperature). This is when the specific heat $C=d E / d T$ is infinite. This corresponds to a density contrast $\mathcal{R}_{\mathrm{CE}}=32.1$ [21. At that point, the system undergoes an isothermal collapse [89. In the case of self-gravitating Brownian particles, this gravitational collapse ultimately leads to a Dirac peak containing all the mass [93]. This structure has an infinite free energy $F \rightarrow-\infty$ (see Appendix B of 92]).

There is a region of ensemble inequivalences in the first region of negative specific heat between $\mathcal{R}_{\mathrm{CE}}=32.1$ and $\mathcal{R}_{\mathrm{MCE}}=709$ (see Fig. 1). The system becomes canonically unstable when the specific heat becomes infinite, passing from positive to negative values, and it becomes microcanonically unstable when the specific heat vanishes, passing from negative to positive values (see Appendix B.

\section{B. Self-gravitating black-body radiation}

The thermodynamics of the self-gravitating blackbody radiation (photon star) confined within a cavity in general relativity has been studied in [162, 163]. Blackbody radiation is equivalent to an ultrarelativistic gas of massless bosons (photons) with a linear equation of state

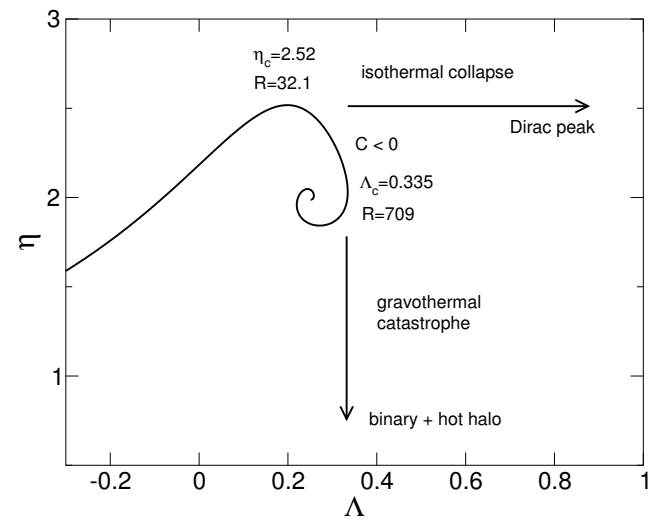

FIG. 1: Caloric curve of the nonrelativistic classical selfgravitating gas.

$P(r)=\epsilon(r) / 3$, where $\epsilon$ denotes the energy density. ${ }^{31}$ The natural dimensionless energy and temperature are

$$
\mathcal{M}=\frac{G M}{R c^{2}}, \quad \mathcal{T}=\frac{k_{B} T_{\infty} G^{1 / 4} R^{1 / 2}}{\hbar^{3 / 4} c^{7 / 4}}
$$

where $\mathcal{E}=M c^{2}$ is the mass-energy and $T_{\infty}$ is the Tolman temperature.

The caloric curve (or series of equilibria) $T_{\infty}(\mathcal{E})$ has the form of a spiral (see Fig. 2 parametrized by the energy density contrast $\mathcal{R}=\epsilon(0) / \epsilon(R) .{ }^{32}$ It was first plotted by Chavanis (see Fig. 15 of 163 ). It is implicit in the work of Sorkin et al. 162 who plotted $\epsilon(R)$ as a function of $M$ in their Fig. 2.

In the microcanonical ensemble there is no equilibrium state above a maximum mass-energy $\mathcal{E}_{\max }=M_{\max } c^{2}$ given by [162, 163 .

$$
\mathcal{M}_{\max }=\frac{G M_{\max }}{R c^{2}}=0.24632 .
$$

Using the Poincaré criterion [64, one can show that the series of equilibria becomes and remains unstable after the first turning point of energy $\mathcal{E}_{\max }$ (a new mode of stability is lost at each turning point of energy). This

31 The equilibrium states of a general relativistic gas described by a linear equation of state of the form $P=q \epsilon$ were considered by Oppenheimer and Volkoff [165, Dmitriev and Kholin [134, Misner and Zapolsky [172, Harrison [173, Harrison et al. [174], Bisnovatyi-Kogan and Zel'dovich [175], Bisnovatyi-Kogan and Thorne 176, Chandrasekhar 177, Yabushita 178 180, Sorkin et al. 162, Schmidt and Homann 181, Chavanis 166, Banks et al. 182, Pesci [183] and Chavanis [163. The coefficient $q=1 / 3$ describes the ultrarelativistic core of neutron stars or the blackbody radiation; the coefficient $q=1$ describes stiff stars [184]; the nonrelativistic isothermal gas is recovered in the limit $q \rightarrow 0$.

32 Basically, the spiral arises because the equation of state of the black-body radiation $P=\epsilon / 3$ is linear, similarly to the linear equation of state $P=\rho k_{B} T / m$ of a nonrelativistic classical gas which also leads to a spiralling caloric curve (see Fig. 17. 


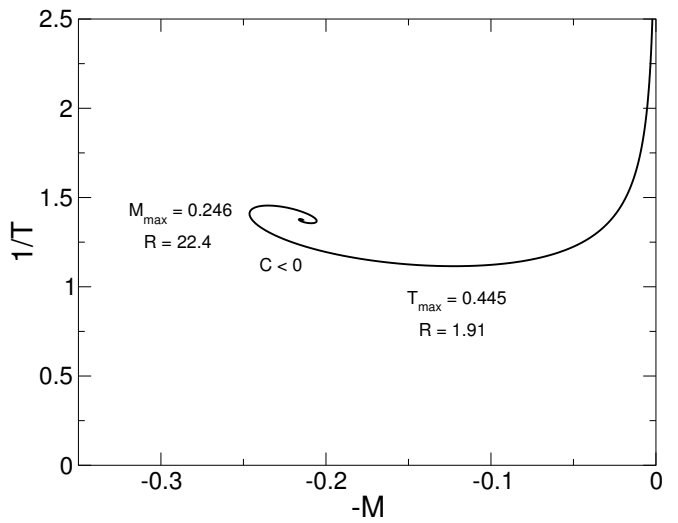

FIG. 2: Caloric curve of the self-gravitating black-body radiation (ultrarelativistic gas) with the normalization from Eq. 23 .

is when the specific heat $C=d \mathcal{E} / d T_{\infty}$ vanishes. This corresponds to a density contrast $\mathcal{R}_{\mathrm{MCE}}=22.4$ 163. In that case, the system is expected to collapse towards a black hole.

In the canonical ensemble there is no equilibrium state above a maximum temperature $\left(T_{\infty}\right)_{\max }$ given by 163

$$
\mathcal{T}_{\max }=\frac{k_{B}\left(T_{\infty}\right)_{\max } G^{1 / 4} R^{1 / 2}}{\hbar^{3 / 4} c^{7 / 4}}=0.445
$$

corresponding to a density contrast $\mathcal{R}_{\mathrm{CE}}=1.91$ [163]. In that case, the system is expected to collapse towards a black hole. As discussed in [163, it is not clear whether the turning point of temperature at $\left(T_{\infty}\right)_{\max }$ along the series of equilibria signals an instability in the present context.

\section{A TYPICAL EXAMPLE: $N=0.1$}

We now consider the thermodynamics of a general relativistic classical gas. Before considering the general case of an arbitrary number of particles, we first treat a typical example where $N$ is neither "too small" nor "too large". We take $N=0.1$ for illustration.

\section{A. Caloric curve}

The caloric curve $\eta(\Lambda)$ is plotted in Fig. 3. It has the form of a double spiral. The spiral on the right, corresponding to low temperatures, will be called the "cold spiral". It occurs at low energies, positive and negative, close to $E=0$. It is a generalization, when the system is relativistic, of the nonrelativistic caloric curve discussed in Sec. III A. It corresponds to weakly relativistic configurations $\left(k_{B} T / m c^{2}\right.$ small $)$. The spiral on the left, corresponding to high temperatures, will be called the

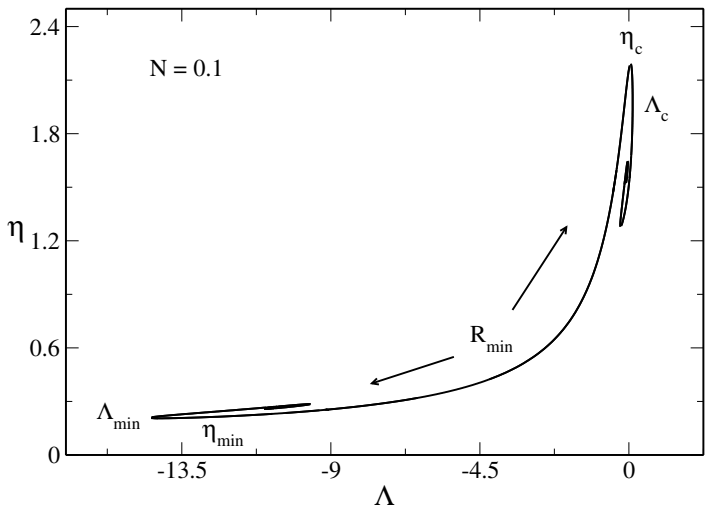

FIG. 3: Typical caloric curve of a classical self-gravitating gas in general relativity (specifically $N=0.1$ ). The arrows indicate the increase of the density contrast $\mathcal{R}=\epsilon(0) / \epsilon(R)$ along the series of equilibria. The system undergoes a gravitational collapse at low energies and low temperatures as in Newtonian gravity (cold spiral, right). It also undergoes a gravitational collapse at high energies and high temperatures as in the case of the self-gravitating radiation (hot spiral, left).

"hot spiral". It occurs at large positive energies (positive energies are allowed because the system is confined within a box). It is related (but not equivalent) to the caloric curve of the ultrarelativistic self-gravitating blackbody radiation discussed in Sec. III B. It corresponds to strongly relativistic configurations $\left(k_{B} T / m c^{2}\right.$ large). For a general relativistic classical gas these two spirals appear at the extremities of the same caloric curve $\eta(\Lambda)$.

The caloric curve presents alternating regions of positive and negative specific heat $C=d E / d T_{\infty}$. This is due to the long-range nature of gravity. The specific heat must be defined with $T_{\infty}$ (the variable conjugate to the energy), not with another temperature like $T(R)$. On the cold spiral, the specific heat is positive before $\eta_{c}$, becomes infinite at $\eta_{c}$, is negative between $\eta_{c}$ and $\Lambda_{c}$, vanishes at $\Lambda_{c}$, is positive again after $\Lambda_{c}$ etc. Similarly, on the hot spiral, the specific heat is positive before $\eta_{\min }$, becomes infinite at $\eta_{\text {min }}$, is negative between $\eta_{\min }$ and $\Lambda_{\text {min }}$, vanishes at $\Lambda_{\text {min }}$, is positive again after $\Lambda_{\text {min }}$ etc.

\section{Microcanonical ensemble}

Let us first consider the microcanonical ensemble where the control parameter is the energy $E$ (or $\Lambda$ ). As in Newtonian gravity [79, 99, 101, there is no global maximum of entropy $S$ at fixed energy $E$ and particle number $N$. However, there exist long-lived metastable states, whose lifetime scales as $e^{N}$, corresponding to local entropy maxima. These metastable states are stable in practice. From Poincaré's theory of linear series of equilibria, we can show that these metastable states are located on the main branch of the caloric curve between $\Lambda_{\text {min }}$ (maximum energy) and $\Lambda_{c}$ (minimum energy). Af- 
ter these turning points of energy (as we progress along the spirals), the equilibrium states are unstable saddle points of entropy. Since the spirals rotate clockwise, a mode of stability is lost at each turning point of energy.

The caloric curve presents the following features (we consider only stable equilibrium states):

(i) Between $\eta_{c}$ and $\Lambda_{c}$ and between $\eta_{\min }$ and $\Lambda_{\min }$, the specific heat $C=d E / d T_{\infty}$ is negative. This is possible in the microcanonical ensemble for systems with long-range interactions because the energy is nonadditive. The specific heat diverges at $\eta_{c}$ and $\eta_{\min }$ and is equal to zero at $\Lambda_{c}$ and $\Lambda_{\text {min }}$. We note that the series of equilibria becomes unstable when the specific heat passes from negative to positive values for the first time. ${ }^{33}$

(ii) For $\Lambda>\Lambda_{c}$, i.e. below a minimum energy, there is no equilibrium state. This is already the case for nonrelativistic systems (see Sec. III A). When its energy is too low, the system experiences a gravothermal catastrophe leading to a binary star surrounded by a hot halo. For sufficiently relativistic systems (corresponding to large values of $N$ ), this collapse may lead to the formation of a black hole. It would be interesting to determine the crossover between these two behaviors.

(iii) For $\Lambda<\Lambda_{\min }$, i.e. above a maximum energy, there is no equilibrium state. This is specific to strongly relativistic systems, like the self-gravitating radiation (see Sec. IIIB. When its energy is too high, the system undergoes a catastrophic collapse towards a black hole.

\section{Canonical ensemble}

We now consider the canonical ensemble where the control parameter is the Tolman temperature $T_{\infty}$ (or $\eta)$. As in Newtonian gravity [79, 99, 101, there is no global minimum of free energy $F$ at fixed particle number $N$. However, there exist long-lived metastable states, whose lifetime scales as $e^{N}$, corresponding to local minima of free energy. These metastable states are stable in practice. From Poincaré's theory of linear series of equilibria, we can show that these metastable states are located on the main branch of the caloric curve between $\eta_{\min }$ (maximum temperature) and $\eta_{c}$ (minimum temperature). After these turning points of temperature (as we progress along the spirals), the equilibrium states are unstable saddle points of free energy. Since the spirals rotate clockwise, a mode of stability is lost at each turning point of temperature.

33 In the microcanonical ensemble the specific heat of stable equilibrium states may be positive or negative. Similarly, unstable configurations may have positive or negative specific heat. Therefore, for systems with long-range interactions like self-gravitating systems, the sign of $C$ is not a criterion of stability in the microcanonical ensemble contrary to the case of systems with shortrange interactions which necessarily have a positive specific heat.

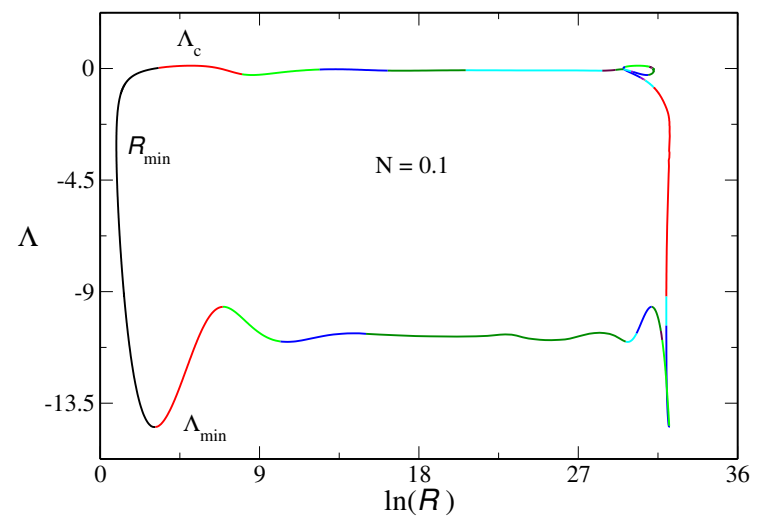

FIG. 4: Dimensionless energy $\Lambda$ as a function of the energy density contrast $\mathcal{R}=\epsilon(0) / \epsilon(R)$ for $N=0.1$.

The caloric curve presents the following features (we consider only stable equilibrium states):

(i) The specific heat is always positive in the canonical ensemble as it should. The specific heat diverges at $\eta_{c}$ and $\eta_{\min }$. We note that the series of equilibria becomes unstable when the specific heat passes from positive to negative values for the first time. ${ }^{34}$

(ii) For $\eta>\eta_{c}$, i.e. below a minimum temperature, there is no equilibrium state. This is already the case for nonrelativistic systems (see Sec. III A). When the system is too cold, it experiences an isothermal collapse leading to a Dirac peak. For sufficiently relativistic systems, the Dirac peak should be replaced by a black hole. It would be interesting to determine the crossover between these two behaviors.

(iii) For $\eta<\eta_{\min }$, i.e. above a maximum temperature, there is no equilibrium state. This is specific to strongly relativistic systems, like the self-gravitating radiation (see Sec. III B). When the system is too hot, it undergoes a catastrophic collapse towards a black hole.

\section{B. Energy density contrast}

In Figs. 4 and 5 we have plotted the dimensionless energy $\Lambda$ and the dimensionless temperature $\eta$ as a function of the energy density contrast $\mathcal{R}=\epsilon(0) / \epsilon(R)$ along the series of equilibria. As for the nonrelativistic gas and for the self-gravitating radiation, the density contrast parametrizes the series of equilibria.

We first observe that there exist a point $\mathcal{R}_{\min }$ at which the density contrast is minimum. Starting from that point, the curves $\Lambda(\mathcal{R})$ and $\eta(\mathcal{R})$ present two branches

\footnotetext{
34 In the canonical ensemble, the specific heat of stable equilibrium states is necessarily positive since it measures the variance of the fluctuations of energy. By contrast, unstable configurations may have positive or negative specific heats.
} 


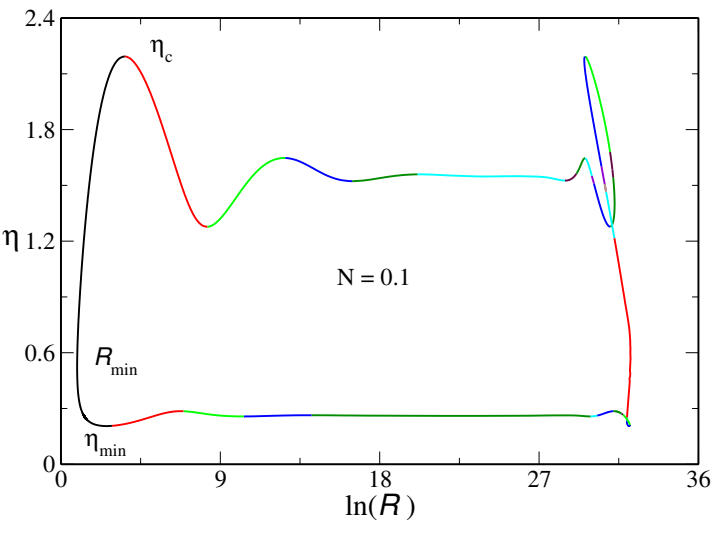

FIG. 5: Dimensionless inverse temperature $\eta$ as a function of the energy density contrast $\mathcal{R}=\epsilon(0) / \epsilon(R)$ for $N=0.1$.

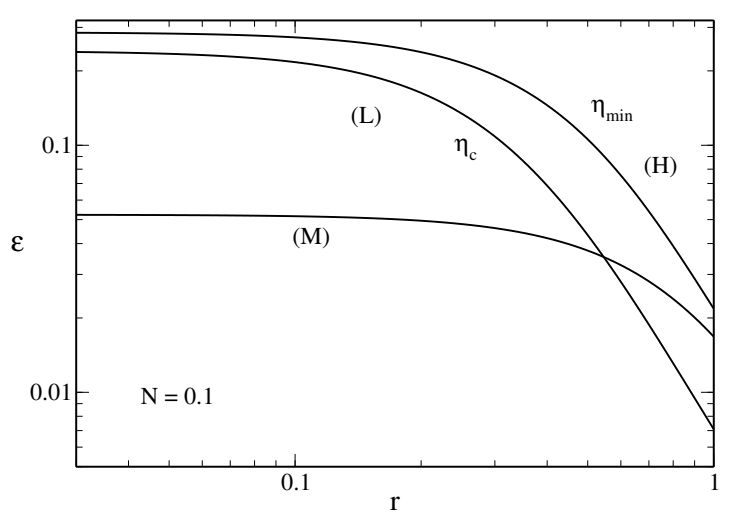

FIG. 6: Energy density profiles $\epsilon(r)$ corresponding to different equilibrium states on the caloric curve of Fig. 3. The curve (M) corresponds to an equilibrium state with a medium energy and a medium temperature. The curve (L) corresponds to an equilibrium state with a low energy and a low temperature (specifically $\eta_{c}$ ). The curve $(\mathrm{H})$ corresponds to an equilibrium state with a high energy and a high temperature (specifically $\eta_{\min }$ ).

which both display damped oscillations at sufficiently high density contrasts. These two branches, and their oscillations, correspond to the two spirals on the caloric curve of Fig. 3. The upper branch (low energies and low temperatures) corresponds to the cold spiral and the lower branch (high energies and high temperatures) corresponds to the hot spiral. ${ }^{35}$

35 We see that, for very high density contrasts, the oscillations are revived and the curves $\Lambda(\mathcal{R})$ and $\eta(\mathcal{R})$ have a complicated behaviour. Furthermore, the two branches merge at a maximum density contrast $\mathcal{R}_{\text {max }}$. This corresponds to the fact that, deep into the spirals, the caloric curve finally goes backwards and superimposes the original curve. Therefore, an equilibrium state on the caloric curve with a given value of energy and temperature $(\Lambda, \eta)$ may correspond to two (or more) configurations with
Actually, it is more physical to study how the density contrast changes with the energy or with the temperature. Starting from $\Lambda\left(\mathcal{R}_{\text {min }}\right)$ in Fig. 4 we see that the density contrast increases monotonically when $\Lambda$ increases towards $\Lambda_{c}$ (i.e. when we reduce the energy) or when $\Lambda$ decreases towards $\Lambda_{\min }$ (i.e. when we increase the energy). Similarly, starting from $\eta\left(\mathcal{R}_{\text {min }}\right)$ in Fig. 5 we see that the density contrast increases monotonically when $\eta$ increases towards $\eta_{c}$ (i.e. when we reduce the temperature) or when $\eta$ decreases towards $\eta_{\min }$ (i.e. when we increase the the temperature). This behavior is illustrated in Fig. 6 showing the energy density profile $\epsilon(r)$ of different equilibrium states with low (L), medium (M), and high $(\mathrm{H})$ energies and temperatures. This figure illustrates the fact that the density contrast increases when, starting from the "middle" point of the caloric curve with the minimum density contrast $\mathcal{R}_{\text {min }}$, we either increase or decrease the energy and the temperature (i.e. we move towards the hot or the cold spiral).

In the microcanonical ensemble, the system becomes unstable after the first turning point of energy on each branch $\left(\Lambda_{c}\right.$ and $\left.\Lambda_{\min }\right)$. In the canonical ensemble, the system becomes unstable after the first turning point of temperature on each branch $\left(\eta_{c}\right.$ and $\left.\eta_{\min }\right)$. Therefore, instability occurs when the system reaches a certain critical concentration: $\mathcal{R}\left(\Lambda_{c}\right)$ and $\mathcal{R}\left(\Lambda_{\text {min }}\right)$ in the microcanonical ensemble; $\mathcal{R}\left(\eta_{c}\right)$ and $\mathcal{R}\left(\eta_{\min }\right)$ in the canonical ensemble.

\section{Central temperature}

In Figs. 7 and 8 we have plotted the normalized energy and the normalized inverse Tolman temperature as a function of the normalized inverse central temperature. We note that the curve $b_{0}(\Lambda)$ is very different from the caloric curve $\eta(\Lambda)$ from Fig. 3. In particular, it does not form spirals. Actually, the curves from Figs. 7 and 8 are more closely related to the curves from Figs. 4 and 5 showing that $b_{0}$ plays a role similar to the density contrast (it parametrizes the series of equilibria). On the other hand, if we restrict ourselves to the stable equilibrium states, we observe on Fig. 8 that the central temperature $T_{0}$ "follows" the Tolman temperature $T_{\infty}$, i.e., $T_{0}$ increases monotonically with $T_{\infty}$ (except in very small intervals close to the critical points). ${ }^{36}$ This is

different density contrasts (see Fig. 35 for an illustration of this phenomenon). However, the configurations with the highest density contrasts are unstable so we will not consider these states in the sequel. For that reason, the left parts of Figs. 4 and 5 will be called "relevant" while the right parts will be called "irrelevant" (see footnote 51). We just consider the relevant parts.

36 This can be understood as follows. We recall that $b_{0}=$ $m c^{2} / k_{B} T_{0}$ and $\eta=G N m^{2} / k_{B} T_{\infty} R$. In the nonrelativistic limit where $T_{0}=T_{\infty}$, we have $\eta=\nu b_{0}$. For $\nu \rightarrow 0$ (see Sec. VI), this linear relationship is valid almost everywhere except for very small values of $\eta$ and $b_{0}$ corresponding to large temperatures where the system is strongly relativistic. For larger values of $\nu$, 


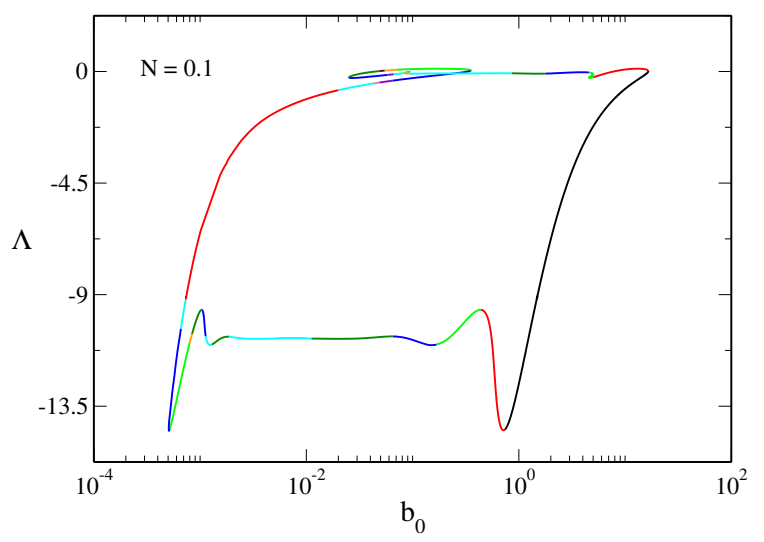

FIG. 7: Normalized energy $\Lambda$ as a function of the inverse central temperature $b_{0}$ for $N=0.1$.

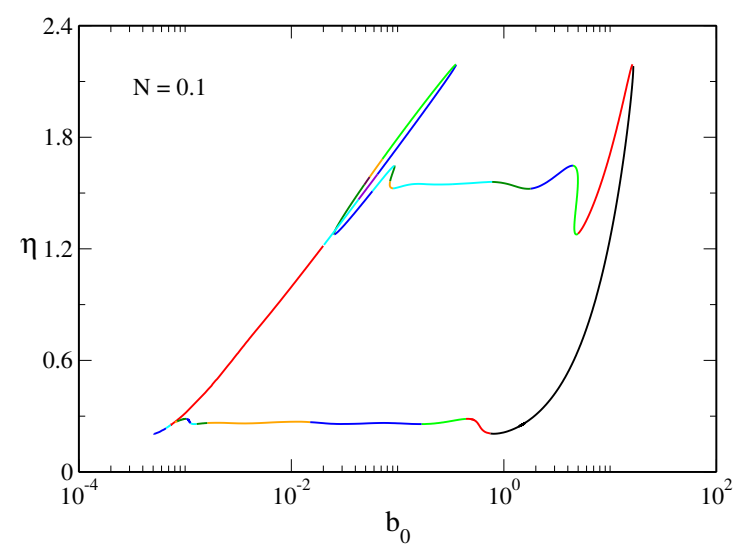

FIG. 8: Normalized inverse temperature $\eta$ as a function of the inverse central temperature $b_{0}$ for $N=0.1$.

different from the energy density contrast that presents a minimum value in the "middle" of the caloric curve. The relation between the evolution of $T_{0}$ and $T_{\infty}$ is more complicated for the unstable equilibrium states but these states are not physically relevant.

\section{Entropy and free energy}

In Fig. 9 we have plotted the entropy $S / N$ as a function of the normalized energy $\Lambda$. As explained in [3, 205], the curve presents some spikes at the extremal energies $\Lambda_{\min }$ and $\Lambda_{c}$. This is because $\delta S=\beta_{\infty} \delta E$ (for a fixed value of $N)$ so that the curves $S\left(\epsilon_{0}\right)$ and $\Lambda\left(\epsilon_{0}\right)$ reach their extremal values at the same points, specified by their central density $\epsilon_{0}$, in the series of equilibria. We also note

the relation between $\eta$ and $b_{0}$ is not linear anymore (see Fig. 8 but it still remains monotonic in the range of stable states.

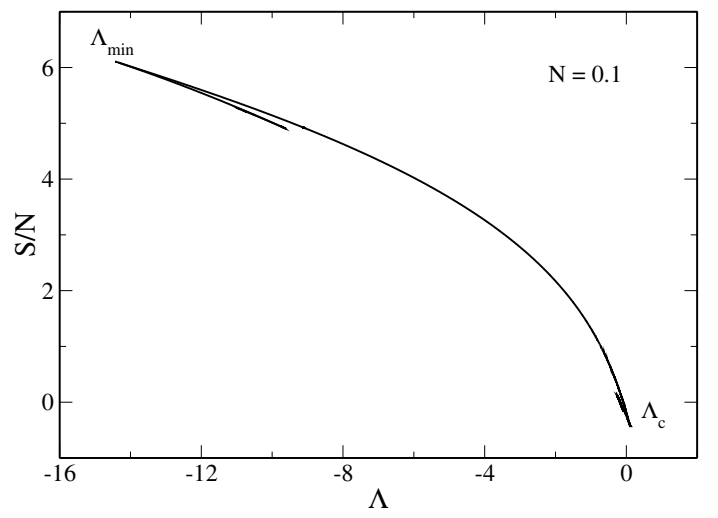

FIG. 9: Entropy per particle $S / N$ as a function of the inverse normalized energy $\Lambda$ for $N=0.1$. We note that the unstable equilibrium states have a lower entropy than the stable equilibrium states. This is of course expected since the stable equilibrium states in the microcanonical ensemble correspond to maxima of entropy at fixed energy and particle number.

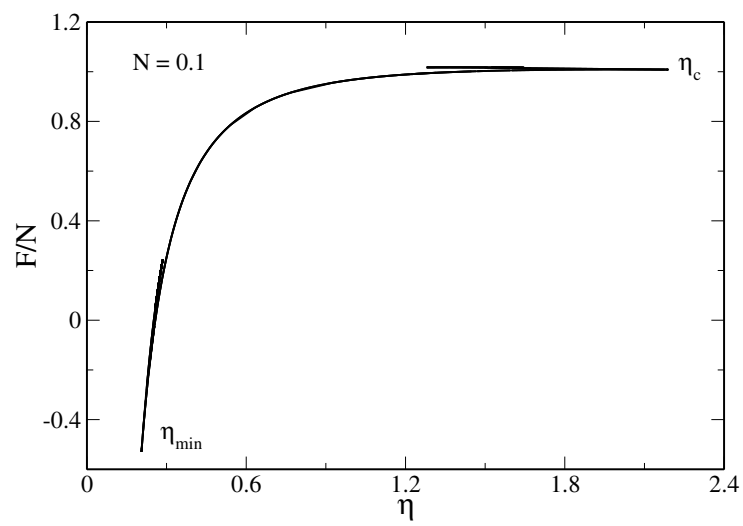

FIG. 10: Free energy per particle $F / N$ as a function of the inverse normalized temperature $\eta$ for $N=0.1$. We note that the unstable equilibrium states have a higher free energy than the stable equilibrium states. This is of course expected since the stable equilibrium states in the canonical ensemble correspond to minima of free energy at fixed particle number.

the presence of two tiny convex dips in the curve $S(\Lambda)$ associated with the two regions of negative specific heats appearing on the caloric curve of Fig. 3 .

In Fig. 10 we have plotted the free energy $F / N$ as a function of the normalized inverse temperature $\eta$. As explained in [3, 205], the curve presents some spikes at the extremal inverse temperatures $\eta_{\min }$ and $\eta_{c}$. This is because $\delta F=-S \delta T_{\infty}$ (for a fixed value of $N$ ) so that the curves $F\left(\epsilon_{0}\right)$ and $\eta\left(\epsilon_{0}\right)$ reach their extremal values at the same points, specified by their central density $\epsilon_{0}$, in the series of equilibria. 


\section{E. Ensembles inequivalence}

From the preceding results, we note that the thermodynamical instabilities occur at different points in the microcanonical and canonical ensembles. Therefore, the statistical ensembles are inequivalent for self-gravitating systems. We can check on the present example the general result according to which "canonical stability implies microcanonical stability" 131. Generally speaking, this is due to the fact that the microcanonical ensemble is more constrained than the canonical ensemble (because of the energy conservation). In the present example, this manifests itself (in relation to the Poincaré criterion) by the fact that the first turning point of temperature occurs before the first turning point of energy in the series of equilibria.

Remark: Statistical ensembles may be inequivalent for systems with long-range interactions because their energy is nonadditive so that the canonical ensemble cannot be deduced from the microcanonical one by considering a subpart of a large system. However, ensembles inequivalence is not compulsory for all systems with long-range interactions. For example, for the HMF model [185 187, the statistical ensembles are equivalent.

\section{F. Dynamical versus thermodynamical stability}

A statistical equilibrium state is thermodynamically stable in the microcanonical ensemble if it is a maximum of entropy at fixed energy and particle number. This corresponds to the "most probable" equilibrium configuration accounting for the constraints of energy and particle number [1, 3]. As we have seen, the equilibrium states on the main branch between $\Lambda_{\text {min }}$ and $\Lambda_{c}$ are thermodynamically stable. They are also dynamically stable with respect to the Vlasov-Einstein equations (governing the evolution of a collisionless star cluster) in view of the general result according to which "thermodynamical stability implies dynamical stability" [130, 131, 155.

Let us now consider the equilibrium states that are not thermodynamically stable.

In Newtonian gravity, it has been shown 124,129 that all isotropic stellar systems with a distribution function of the form $f=f(\epsilon)$ with $f^{\prime}(\epsilon)<0$ are dynamically stable with respect to the Vlasov-Poisson equations. This implies, in particular, that all the equilibrium states on the caloric curve of Fig. 1 are dynamically stable, even those that are thermodynamically unstable (e.g., those deep in the spiral).

By contrast, in general relativity, there is a conjecture by Ipser [155] according to which thermodynamically unstable states are also dynamically unstable, so that thermodynamical and dynamical stability actually coincide.
If this conjecture is confirmed, ${ }^{37}$ we can conclude that the equilibrium states located after the turning points of energy (as we progress along the spirals) in the caloric curve of Fig. 3 are both thermodynamically and dynamically unstable.

In order to reconcile these apparently contradictory results we have to consider the timescale of the instability. It is very likely that the time to develop the dynamical instability diverges as the level of relativity tends to zero. Therefore, it is very long for weakly relativistic systems (becoming comparable to the collisional relaxation time) and decreases for strongly relativistic systems. Since, for small or moderate values of $N$, the cold spiral is made of weakly relativistic configurations, we conclude that the instability at the minimum energy $E_{c}$ is essentially a thermodynamical (slow/secular) instability. By contrast, since the hot spiral is made of strongly relativistic configurations, we conclude that the instability at the maximum energy $E_{\max }$ is essentially a dynamical (fast) instability.

The previous results apply to isolated systems in the microcanonical ensemble. If we now consider the case of systems in contact with a thermal bath in the canonical ensemble we get different results. A statistical equilibrium state is thermodynamically stable in the canonical ensemble if it is a minimum of free energy at fixed particle number. On the other hand, it can be shown that thermodynamical stability in the canonical ensemble coincides with dynamical stability with respect to the EulerPoisson equations in Newtonian gravity [3, 89, 188, and with respect to the Euler-Einstein equations in general relativity [189 192 (governing the evolution of a gaseous star). Therefore, the equilibrium states on the main branch between $\eta_{\min }$ and $\eta_{c}$ are both thermodynamically and dynamically stable. They cease to be stable (thermodynamically and dynamically) after the turning points of temperature.

\section{GENERAL CASE}

We now describe the caloric curves of a general relativistic self-gravitating classical gas for an arbitrary number of particles $N$. As the sphere becomes more compact ( $N$ or $\nu$ increases) general relativistic effects are more intense.

37 As recalled in the Introduction the equivalence between dynamical and thermodynamical stability in general relativity has been checked numerically for several distribution functions including heavily truncated isothermal distributions. It would be interesting to know if it remains valid for box-confined isothermal spheres. 


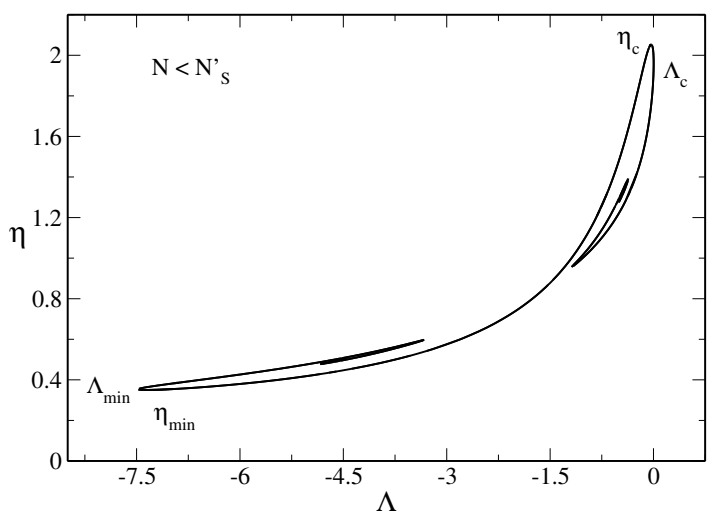

FIG. 11: Caloric curve for $N<N_{S}^{\prime}=0.128$ (specifically $N=0.125)$.

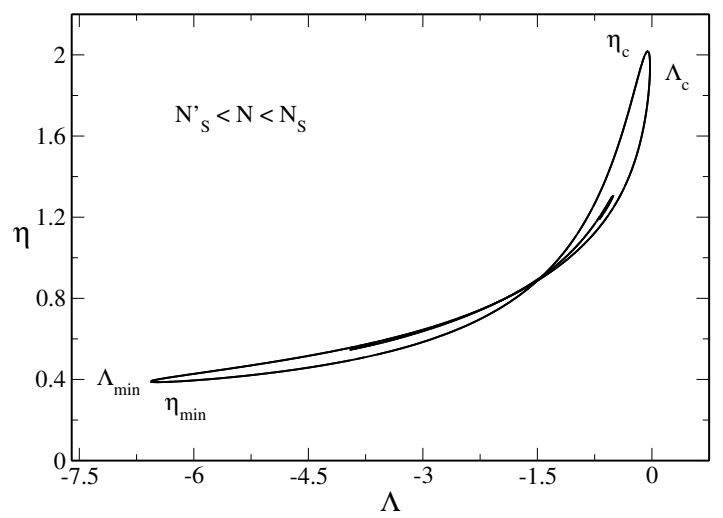

FIG. 12: Caloric curve for $N_{S}^{\prime}=0.128<N<N_{S}=0.1415$ (specifically $N=0.13$ ).

\section{A. The case $N<N_{S}^{\prime}$}

The case $N<N_{S}^{\prime}=0.128$ corresponds to the typical example treated in Sec. IV ${ }^{38}$ The caloric curve has the form of a double spiral (see Fig. 3).

When $N \rightarrow 0$, the hot spiral is pushed towards infinity $\left(\Lambda_{\min } \rightarrow-\infty\right.$ and $\left.\eta_{\min } \rightarrow 0\right)$ and the rest of the caloric curve, consisting in the main branch and the cold spiral, tends to a limit curve corresponding to the nonrelativistic caloric curve of Fig. 11. The limit $N \rightarrow 0$ is studied in detail in Sec. VI

Inversely, as $N$ increases, the two spirals approach each other. For $N<N_{S}^{\prime}$, they remain clearly separated (see Fig. 11.

38 The existence and the value of the critical particle numbers $N_{S}^{\prime}$, $N_{S}$ and $N_{\max }$ (see below) can be understood from simple graphical constructions as explained in Appendix C

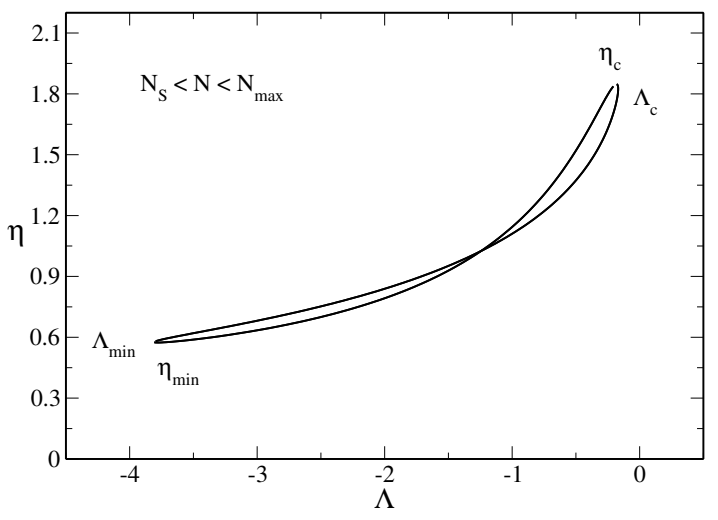

FIG. 13: Caloric curve for $N_{S}=0.1415<N<N_{\max }=$ 0.1764 (specifically $N=0.15$ ).

B. The case $N_{S}^{\prime}<N<N_{S}$

For $N_{S}^{\prime}=0.128<N<N_{S}=0.1415$, the caloric curve still presents two spirals but the difference with the previous case is that the spirals are amputed (truncated) and touch each other (see Fig. 12).

\section{The case $N_{S}<N<N_{\max }$}

For $N_{S}=0.1415<N<N_{\max }=0.1764$, the caloric curve has the form of a single loop resembling the symbol $\infty$ (see Fig. 13). As $N$ increases further, the loop shrinks more and more until it reduces to a point $\left(\Lambda_{*}, \eta_{*}\right)=$ $(-0.9829,1.2203)$ when $N=N_{\max }$. For $N>N_{\max }$, no equilibrium state is possible.

\section{The critical point $N=N_{\max }$}

The case $N=N_{\max }=0.1764$, is very special because an equilibrium state exists only at a unique energy $\Lambda_{*}=-0.9829$ and a unique temperature $\eta_{*}=1.2203$. This equilibrium state is probably unstable or, at best, marginally stable. Despite this very peculiar circumstance, the energy density and temperature profiles are regular (see Fig. 14).

\section{E. The case $N>N_{\max }$}

Equilibrium states may exist only below a maximum particle number $N_{\max }=0.1764$. For $N>N_{\max }$ the system is expected to collapse and form a black hole whatever the value of its energy and temperature. Coming back to dimensional variables, the inequality $N \leq$ $N_{\max }=0.1764$ required for the existence of an equilib- 


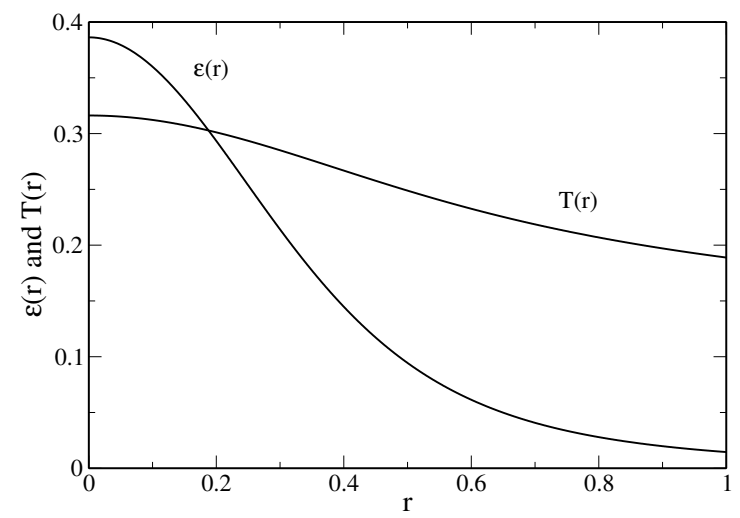

FIG. 14: Energy density profile $\epsilon(r)$ and temperature profile $T(r)$ at the critical point $N=N_{\max }=0.1764$.

rium state can be written as

$$
\nu=\frac{G N m}{R c^{2}} \leq \nu_{\max }=0.1764 .
$$

For a given box radius $R$, there is no statistical equilibrium state if $N$ is larger than

$$
N_{\text {max }}=0.1764 \frac{R c^{2}}{G m} .
$$

Alternatively, for a given particle number $N$ there is no equilibrium state if the radius is smaller than

$$
R_{\min }=5.67 \frac{G N m}{c^{2}} .
$$

The minimum radius $R_{\min }$ is similar to the Schwarzschild radius $R_{S}=2 G M / c^{2}$ except that it is defined in terms of the rest mass $N m$ instead of the mass-energy $M$. Similarly, the maximum particle number $N_{\max }$ is similar to a Schwarzschild particle number $N_{S}=R c^{2} / 2 G m$, where $R$ is the box radius. We note that, formally, Eq. (28) satisfies the Buchdahl 193 inequality $R \geq(9 / 8) R_{S}$.

\section{F. Physical caloric curve}

As we approach $N_{\max }=0.1764$ the series of equilibria $\eta(\Lambda)$ becomes very complex. However, this complexity is only apparent as it essentially concerns the unstable equilibrium states that are not physically relevant. If we focus on the stable (actually metastable) equilibrium states, we just need to consider the main branch of the series of equilibria. This defines the physical caloric curve. This curve always has the same shape. In the microcanonical ensemble it extends from $\Lambda_{\min }$ to $\Lambda_{c}$. In the canonical ensemble it extends from $\eta_{\min }$ to $\eta_{c}$. The only effect of increasing $N$ is to make this branch smaller and smaller until it completely disappears at $N_{\max }$. In this sense, the interpretation of the caloric curve given in Sec. IV] is valid for any $N \leq N_{\max }$. Since the physical caloric curve shrinks when the compactness parameter $\nu$ increases, this implies that general relativistic effects render the system more unstable. This feature will be studied in more detail in the following sections.

\section{THE LIMIT $N \rightarrow 0$}

In this section, we study the form of the caloric curve in the limit $N \rightarrow 0$. If we come back to dimensional variables, this corresponds to $\nu=G N m / R c^{2} \rightarrow 0$ which can be written as $N \ll N_{\max }$ or $R \gg R_{\min }$. We successively consider the cold spiral and the hot spiral.

\section{A. The cold spiral}

When $N \rightarrow 0$, the caloric curve $\eta(\Lambda)$ tends to a limit curve corresponding to the caloric curve of a nonrelativistic classical self-gravitating gas (see Fig. 11). The hot spiral is rejected at infinity $\left(\Lambda_{\min } \rightarrow-\infty\right.$ and $\left.\eta_{\min } \rightarrow 0\right)$ as discussed in Sec. VII.

In Figs. 15 and 16, using the dimensionless variables $\eta$ and $\Lambda$, we show how the caloric curve $\eta(\Lambda)$ evolves with $N$. Starting from the nonrelativistic caloric curve corresponding to $N \rightarrow 0$, and increasing $N$, we see that the minimum normalized energy and the minimum normalized temperature of the cold spiral both increase $\left(\Lambda_{c}\right.$ and $\eta_{c}$ decrease) as $N$ increases. Therefore, increasing $N$, i.e. increasing the compactness parameter $\nu$, advances the destabilization of the system in the microcanonical and canonical ensembles at low energies and low temperatures. Therefore, general relativistic effects render the system more unstable. Indeed, the instability occurs sooner than in the nonrelativistic limit.

This result, obtained from a fully general relativistic treatment, can be compared with the former result obtained in 166 from a semirelativistic treatment in which the gas is described by a relativistic equation of state while gravity is described by Newton's theory. In that case, as shown in Fig. 1 of [166], the critical energy increases with $N$ as in the present work but the critical temperature remains constant $\left(\Lambda_{c}\right.$ decreases while $\eta_{c}$ remains equal to 2.52). Therefore, the semi relativistic treatment of [166] does not correcty account for the increase of the critical temperature when gravity is described by Einstein's theory.

In Fig. 17, we have plotted the normalized energy density profile at $\eta_{c}$ for different values of $N$. For $N \rightarrow 0$, we recover the nonrelativistic isothermal profile. As $N$ increases, the energy density profiles remain substantially the same.

In Fig. 18, we have plotted the normalized temperature profile at $\eta_{c}$ for different values of $N$. For $N \rightarrow 0$, the temperature becomes uniform as it has to be for a Newtonian self-gravitating system at statistical equilibrium. As $N$ increases, we see that the temperature becomes spatially inhomogeneous even though the system 


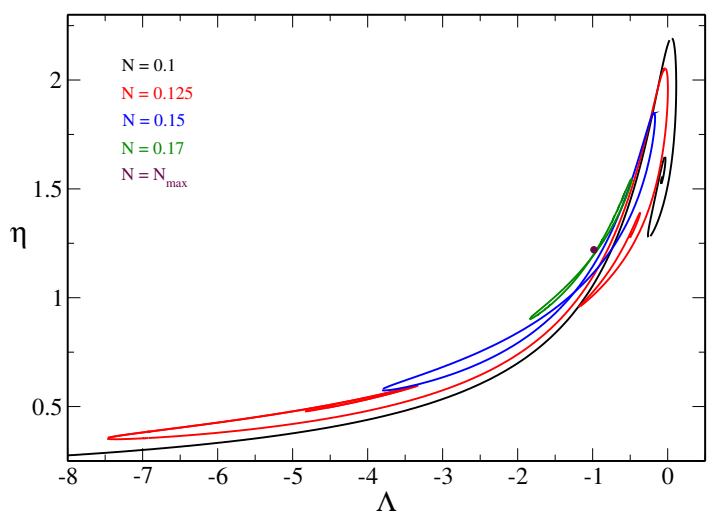

FIG. 15: Caloric curve $\eta(\Lambda)$ for different values of $N$ (specifically $\left.N=0.1,0.125,0.15,0.17, N_{\max }\right)$. A zoom of the cold spiral for small values of $N$ is provided in Fig. 16 .

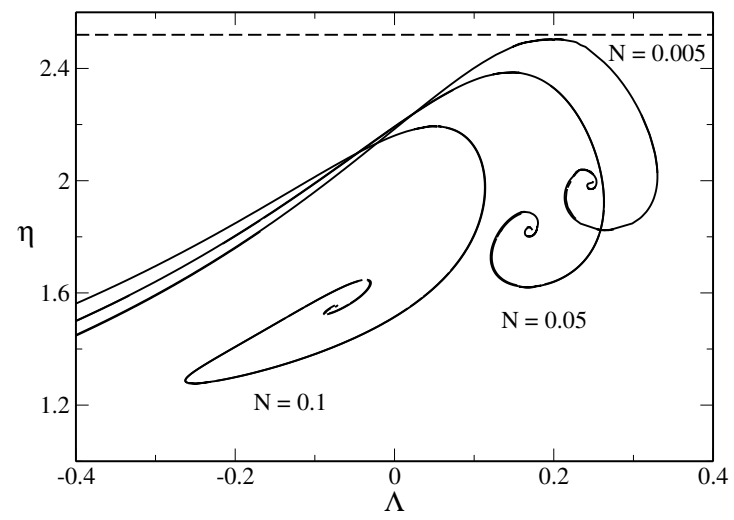

FIG. 16: Evolution of the cold spiral as $N$ increases, starting from the nonrelativistic spiral $(N \rightarrow 0)$. Relativistic effects increase the critical energy and the critical temperature, making the system less stable. The dashed line corresponds to $\eta_{c}=2.52$ (see the discussion in the main text).

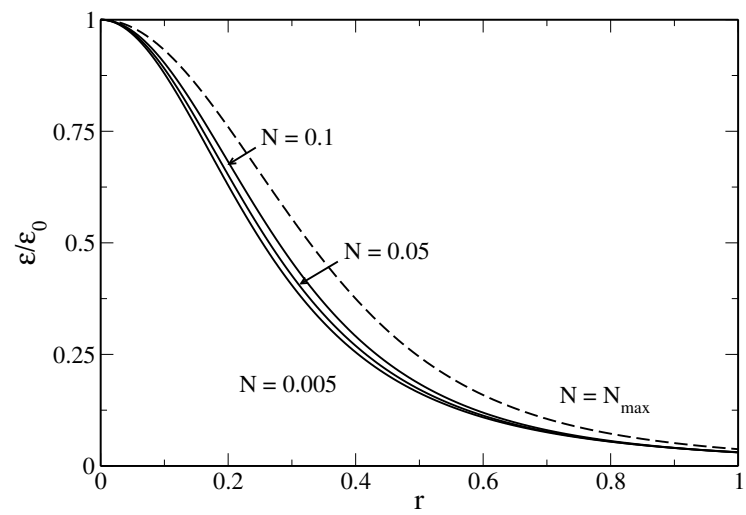

FIG. 17: Normalized energy density profiles at $\eta_{c}$ for different values of $N$. The values of the central densities are $\epsilon_{0}=1.28 \times 10^{-2}(N=0.005), \epsilon_{0}=0.123(N=0.05)$, and $\epsilon_{0}=0.235(N=0.1)$. We have added in dashed line the energy density profile at $N=N_{\max }\left(\epsilon_{0}=0.386\right)$ for comparison.

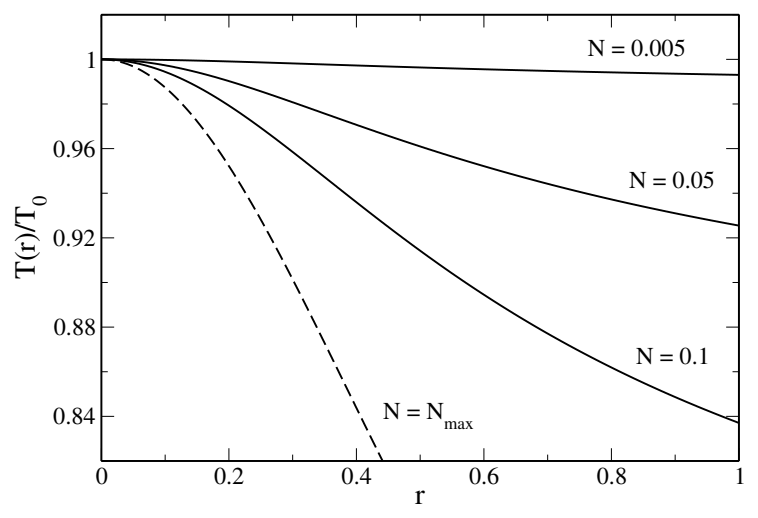

FIG. 18: Normalized temperature profiles at $\eta_{c}$ for different values of $N$. The values of the central temperatures are $T_{0}=$ $2.02 \times 10^{-3}(N=0.005), T_{0}=2.39 \times 10^{-2}(N=0.05)$, and $T_{0}=6.09 \times 10^{-2}(N=0.1)$. We have added in dashed line the temperature profile at $N=N_{\max }\left(T_{0}=0.316\right)$ for comparison.

is at statistical equilibrium. This is a manifestation of the Tolman effect [164] in general relativity.

\section{B. The hot spiral}

For $N \rightarrow 0$, we have seen that the hot spiral of the caloric curve $\eta(\Lambda)$ is rejected at infinity $\left(\eta_{\min } \rightarrow 0\right.$ and $\left.\Lambda_{\text {min }} \rightarrow-\infty\right)$. In order to investigate the evolution of the hot spiral in the limit $N \rightarrow 0$ accurately, we need to introduce a different normalization of $E$ and $T_{\infty}$. Indeed, the normalized variables $\Lambda$ and $\eta$ defined by Eq. (17) are adapted to the nonrelativistic limit $k_{B} T \ll m c^{2}$ (cold spiral) when $\nu \rightarrow 0$. As shown in [1], the normalized variables adapted to the ultrarelativistic limit $k_{B} T \gg$ $m c^{2}$ (hot spiral) when $\nu \rightarrow 0$ are

$$
\mathcal{M} \equiv \frac{G M}{R c^{2}}=-\Lambda \nu^{2}+\nu, \quad \mathcal{B} \equiv \frac{R c^{4}}{G N k_{B} T_{\infty}}=\frac{\eta}{\nu^{2}}
$$

Using the dimensionless variables introduced in Appendix $\mathrm{B}$ of [2], we get

$$
\mathcal{M} \equiv M=-\Lambda N^{2}+N, \quad \mathcal{B} \equiv \frac{\beta_{\infty}}{N}=\frac{\eta}{N^{2}} .
$$

The first quantity represents the mass-energy $M$ and the second quantity represents the inverse Tolman temperature $\beta_{\infty}$ divided by the particle number $N .^{39}$ When $N \rightarrow 0$, the caloric curve $\mathcal{B}(\mathcal{M})$ tends to a limit curve corresponding to the caloric curve of an ultrarelativistic

39 By contrast, $\Lambda=-(M-N m) c^{2} R / G N^{2} m^{2}$ (or $\Lambda=-(M-$ $N) / N^{2}$ ) represents the binding energy divided by $N^{2}$ and $\eta=$ $\beta_{\infty} G N m^{2} / R$ (or $\eta=\beta_{\infty} N$ ) represents the inverse Tolman temperature $\beta_{\infty}=1 /\left(k_{B} T_{\infty}\right)$ multiplied by $N$. 


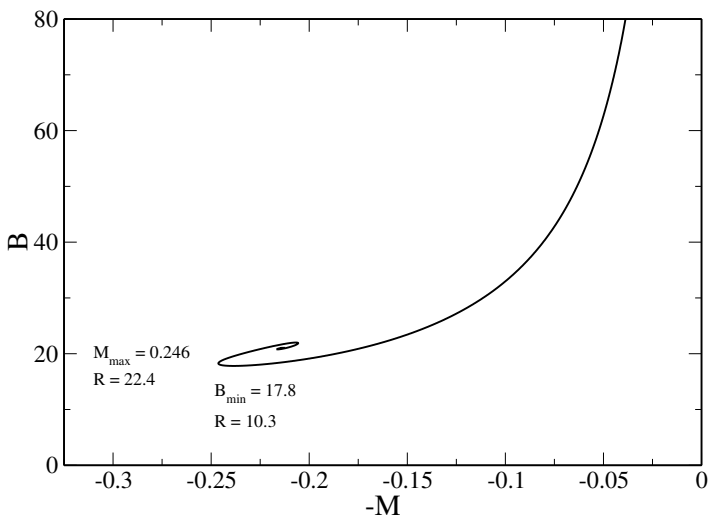

FIG. 19: Caloric curve $\mathcal{B}(\mathcal{M})$ of the ultrarelativistic selfgravitating classical gas when $N \rightarrow 0$. The manner to obtain this asymptotic curve is explained in [1].

classical self-gravitating gas (see Fig. 19 [1]. The cold spiral is rejected at infinity $\left(\mathcal{M}_{c} \rightarrow 0\right.$ and $\left.\mathcal{B}_{c} \rightarrow+\infty\right)$ as discussed in Sec. VII. The caloric curve $\mathcal{B}(\mathcal{M})$ of Fig. 19 is similar, but not equivalent, to the caloric curve $1 / \mathcal{T}(\mathcal{M})$ of the self-gravitating black-body radiation represented in Fig. 2 (see 1 for a detailed discussion of the analogies and differences between the two caloric curves). The maximum mass and the corresponding energy density contrast of the hot spiral when $N \rightarrow 0$ are [1]

$$
\mathcal{M}_{\text {max }}=\frac{G M_{\max }}{R c^{2}}=0.24632, \quad \mathcal{R}_{c}=22.4 .
$$

These are the same values as for the self-gravitating black-body radiation [162, 163] (see Sec. IIIB). On the other hand, the maximum temperature and the corresponding energy density contrast of the hot spiral when $N \rightarrow 0$ are [1]

$$
\mathcal{B}_{\min } \equiv \frac{R c^{4}}{G N k_{B}\left(T_{\infty}\right)_{\max }}=17.809, \quad \mathcal{R}_{c}^{\prime}=10.3
$$

These values differ from those of the self-gravitating black-body radiation (see Sec. IIIB) for the reasons explained in 1 .

In Figs. 20 and 21, using the dimensionless variables $\mathcal{B}$ and $\mathcal{M}$, we show how the caloric curve $\mathcal{B}(\mathcal{M})$ evolves with $N$. Starting from the ultrarelativistic caloric curve corresponding to $N \rightarrow 0$, and increasing $N$, we see that the maximum mass $\mathcal{M}_{\text {max }}$ first slightly increases before decreasing while the maximum normalized temperature always decreases $\left(\mathcal{B}_{\min }\right.$ increases). Therefore, except for very small values of $N$, increasing $N$, i.e. increasing the compactness parameter $\nu$, advances the destabilization of the system in the microcanonical and canonical ensembles at high energies and high temperatures. Therefore, general relativistic effects render the system more unstable. Indeed, the instability occurs sooner than in

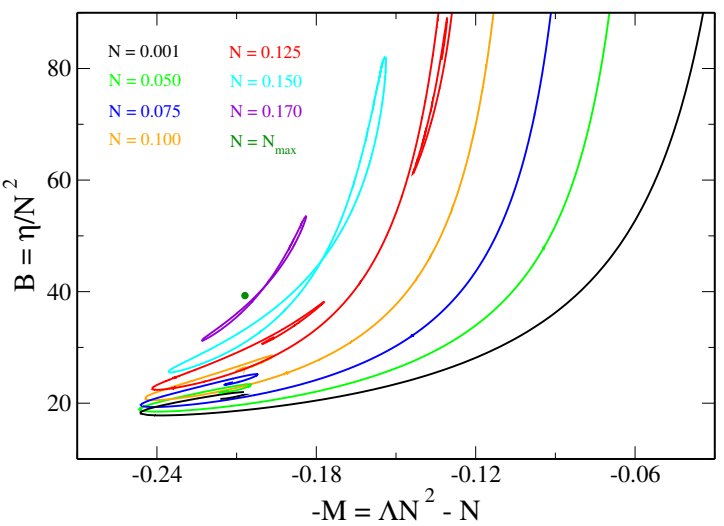

FIG. 20: Caloric curves $\mathcal{B}(\mathcal{M})$ for different values of $N$ (specifically $\left.N=0.001,0.05,0.075,0.1,0.125,0.15,0.17, N_{\max }\right) . \quad \mathrm{A}$ zoom of the hot spiral is provided in Fig. 21.

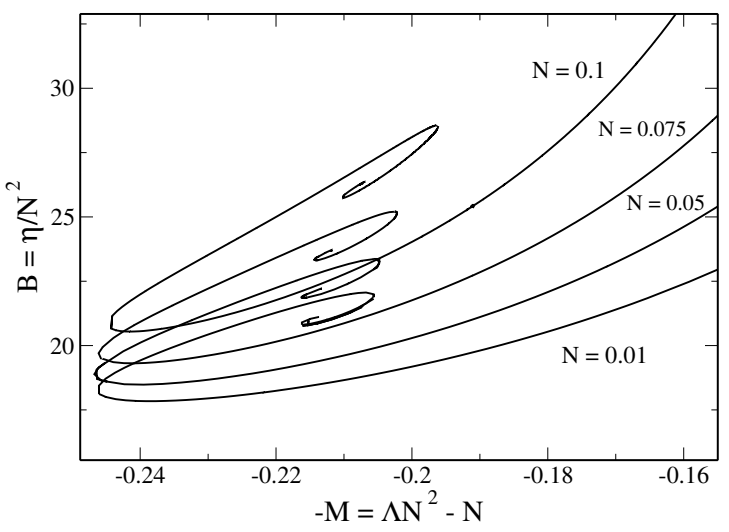

FIG. 21: Evolution of the hot spiral as $N$ increases, starting from the ultrarelativistic spiral $(N \rightarrow 0)$. Except for very small values of $N$, increasing $N$ decreases the critical energy and the critical temperature making the system less stable.

the ultrarelativistic limit. ${ }^{40}$ We also note that $\mathcal{M} \ll 1$ so that isothermal spheres are always far from the black hole (Schwarzschild) limit $\mathcal{M}_{S}=1 / 2$.

\section{EVOLUTION OF THE CRITICAL POINTS WITH $N$}

In this section, we study how the critical points of the caloric curve $\eta(\Lambda)$ identified in the preceding sections evolve with the particle number $N$.

40 It is important to realize that when the compactness parameter $\nu$ increases the system is less relativistic on the hot spiral from the viewpoint of kinematics since the ultrarelativistic limit corresponds to $\nu \rightarrow 0$. Therefore, the system is more general relativistic but less special relativistic. 


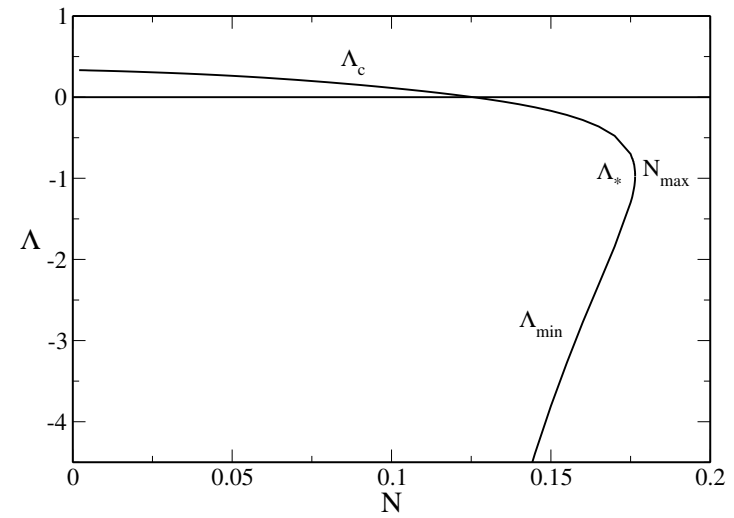

FIG. 22: Evolution of the critical energies $\Lambda_{c}$ and $\Lambda_{\text {min }}$ with $N$.

\section{A. Energy and temperature}

In Fig. 22 we have plotted the evolution of the critical energies $\Lambda_{c}$ and $\Lambda_{\text {min }}$, corresponding to the cold and hot spirals, as a function of $N$. For $N \rightarrow 0$, we find that $\Lambda_{c}$ tends to the Newtonian value 0.335 58 (see Sec. III A) while $\Lambda_{\min }$ tends to $-\infty$ as (see Sec. VIB)

$$
\Lambda_{\min } \sim-\frac{0.24632-N}{N^{2}} .
$$

As $N$ increases, we see on Fig. 22 that $\Lambda_{c}$ decreases monotonically while $\Lambda_{\text {min }}$ increases monotonically. For $N \rightarrow N_{\max }=0.1764$, they tend to the common value

$$
\Lambda_{*}=-0.9829 .
$$

Close to the maximum particle number, we have the scaling law ${ }^{41}$

$$
\Lambda_{X}-\Lambda_{*} \sim \pm 6.7\left(N_{\max }-N\right)^{1 / 2}
$$

where $\Lambda_{X}$ stands for $\Lambda_{c}$ or $\Lambda_{\text {min }}$. We note on Fig. 22 that $\Lambda_{\min }$ is always negative while $\Lambda_{c}$ is positive for $N<N_{b}=0.1255$ and negative for $N>N_{b}=0.1255$. A negative value of $\Lambda$, i.e. a positive energy $E$, is usually impossible because the system would explode and disperse away. ${ }^{42}$ However, in our case, stable equilibrium states with positive energies are possible because

41 Here and in the following, the prefactors are given at an indicative level because the numerics is not very accurate close to $N_{\max }$.

42 In Newtonian gravity, there is no equilibrium state for stellar systems with positive energy. Indeed, according to the equilibrium virial theorem $2 K+W=0$ we have $E=K+W=(1 / 2) W=$ $-K<0$. In general relativity, equilibrium states of star clusters may have a positive energy. This observation was first made by Zel'dovich [194]. However, when the system is unbounded, the equilibrium states with $E>0$ are usually unstable.

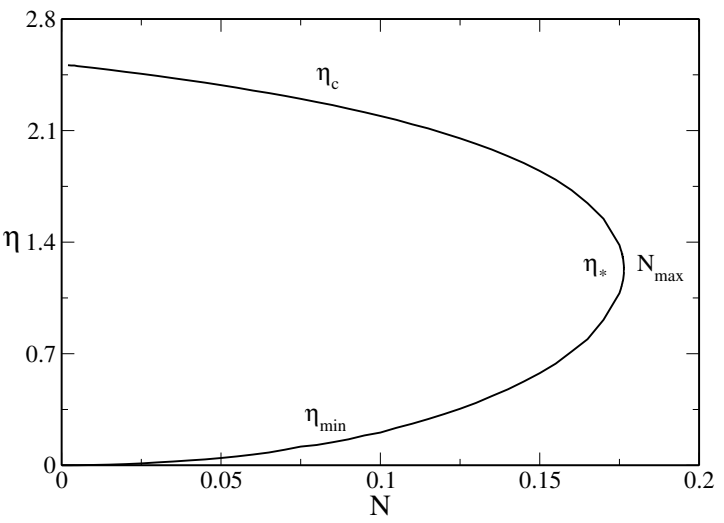

FIG. 23: Evolution of the critical inverse temperatures $\eta_{c}$ and $\eta_{\min }$ with $N$.

we work inside a box so that the system is confined by the walls of the box.

In Fig. 23 we have plotted the evolution of the critical temperatures $\eta_{c}$ and $\eta_{\text {min }}$, corresponding to the cold and hot spirals, as a function of $N$. For $N \rightarrow 0$, we find that $\eta_{c}$ tends to the Newtonian value 2.52 21. (see Sec. III A) while $\eta_{\min }$ tends to zero as (see Sec. VIB

$$
\eta_{\min } \sim 17.809 N^{2}
$$

As $N$ increases, we see on Fig. 23 that $\eta_{c}$ decreases monotonically while $\eta_{\min }$ increases monotonically. For $N \rightarrow N_{\max }=0.1764$, they tend to the common value

$$
\eta_{*}=1.2203 .
$$

Close to the maximum particle number, we have the scaling law

$$
\eta_{X}-\eta_{*} \sim \pm 3.9\left(N_{\max }-N\right)^{1 / 2}
$$

where $\eta_{X}$ stands for $\eta_{c}$ or $\eta_{\min }$.

As explained in Sec. VIB, in order to study the evolution of the hot spiral with $N$, it is better to use the dimensionless variables $\mathcal{M}$ and $\mathcal{B}$.

In Fig. 24 we have plotted the evolution of the critical masses $\mathcal{M}_{c}$ and $\mathcal{M}_{\max }$, corresponding to the cold and hot spirals, as a function of $N$. For $N \rightarrow 0, \mathcal{M}_{c}$ tends to zero as (see Sec. VIA)

$$
\mathcal{M}_{c} \simeq N-0.335 N^{2}
$$

while $\mathcal{M}_{\max }$ tends to the ultrarelativistic value 0.24632 [1] coinciding with the value obtained in Refs. [162, 163] for the self-gravitating black-body radiation (see Secs. III B and VIB. As $N$ increases, we see on Fig. 24 that $\mathcal{M}_{c}$ increases monotonically while $\mathcal{M}_{\max }$ first increases (zoom not shown), reaches a maximum value 0.24642 at $N=0.0418(137)$, and decreases. For $N \rightarrow N_{\max }=$ 0.1764 , they tend to the common value

$$
\mathcal{M}_{*}=0.20703 \text {. }
$$




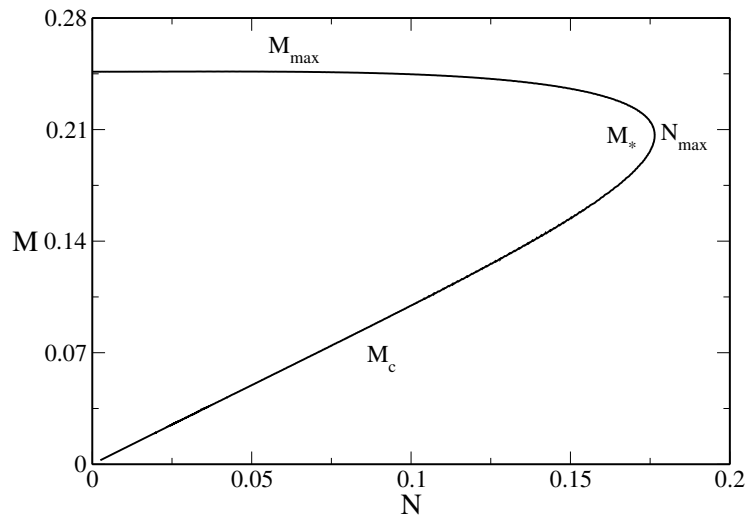

FIG. 24: Evolution of the critical masses $\mathcal{M}_{c}$ and $\mathcal{M}_{\max }$ with $N$.

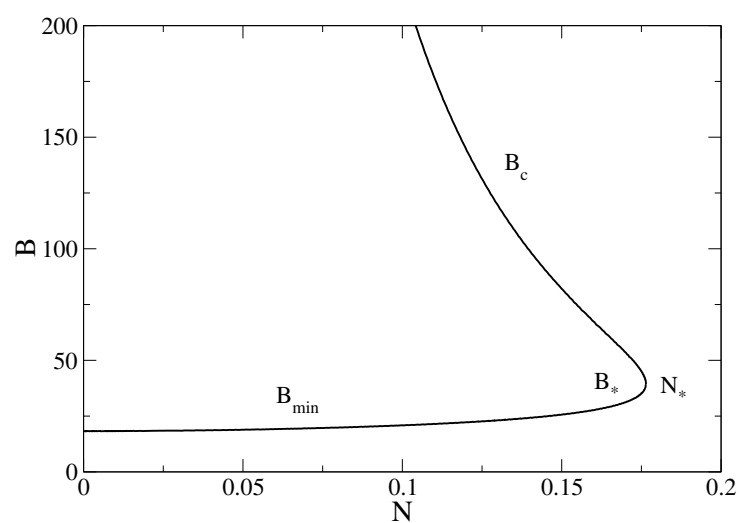

FIG. 25: Evolution of the critical inverse temperatures $\mathcal{B}_{c}$ and $\mathcal{B}_{\text {min }}$ with $N$.

Close to the maximum particle number, we have the scaling law

$$
\mathcal{M}_{X}-\mathcal{M}_{*} \sim \pm 0.24\left(N_{\max }-N\right)^{1 / 2}
$$

where $\mathcal{M}_{X}$ stands for $\mathcal{M}_{\max }$ or $\mathcal{M}_{c}$.

In Fig. 25 we have plotted the evolution of the critical temperatures $\mathcal{B}_{c}$ and $\mathcal{B}_{\text {min }}$, corresponding to the cold and hot spirals, as a function of $N$. For $N \rightarrow 0, \mathcal{B}_{c}$ tends to $+\infty$ as (see Secs. III A and VIB

$$
\mathcal{B}_{c} \sim \frac{2.52}{N^{2}}
$$

while $\mathcal{B}_{\text {min }}$ tends to the ultrarelativistic value 17.809 [1] (see Sec. VIB]. As explained in [1], this value is different from the value of the maximum temperature obtained for the self-gravitating black-body radiation (see Sec. III B). As $N$ increases, we see on Fig. 25 that $\mathcal{B}_{c}$ decreases monotonically while $\mathcal{B}_{\min }$ increases monotonically. For $N \rightarrow N_{\max }=0.1764$, they tend to the common value

$$
\mathcal{B}_{*}=39.1918 \text {. }
$$

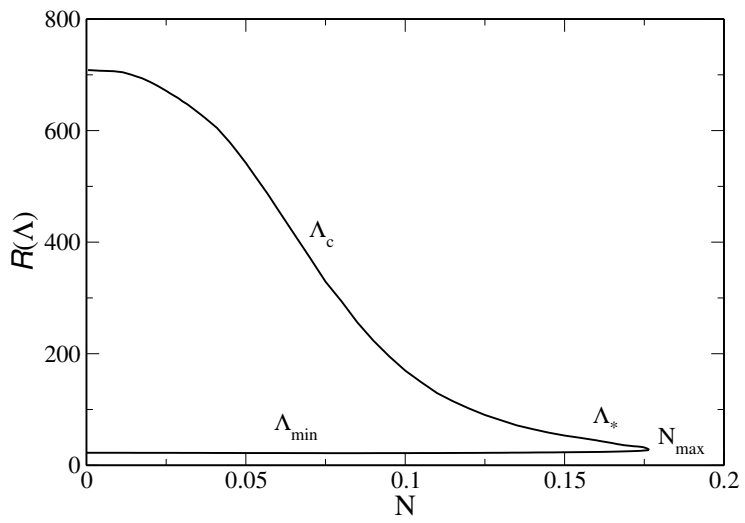

FIG. 26: Energy density contrast at the microcanonical collapse energies $\Lambda_{c}$ and $\Lambda_{\min }$ as a function of $N$.

Close to the maximum particle number, we have the scaling law

$$
\mathcal{B}_{X}-\mathcal{B}_{*} \sim \pm 130\left(N_{\max }-N\right)^{1 / 2}
$$

where $\mathcal{B}_{X}$ stands for $\mathcal{B}_{\min }$ or $\mathcal{B}_{c}$.

\section{B. Density contrast}

In Fig. 26 we have plotted the evolution of the energy density contrast $\mathcal{R}=\epsilon(0) / \epsilon(R)$ at the critical energies $\Lambda_{c}$ and $\Lambda_{\min }$ as a function of $N$. For $N \rightarrow 0$, we find that $\mathcal{R}\left(\Lambda_{c}\right)$ tends to the Newtonian value 709 [57] (see Sec. IIIA while $\mathcal{R}\left(\Lambda_{\text {min }}\right)$ tends to the ultrarelativistic value 22.41 coinciding with the value obtained in Ref. 163 for the self-gravitating black-body radiation (see Secs. III B and VIB). As $N$ increases, $\mathcal{R}\left(\Lambda_{c}\right)$ decreases monotonically. On the other hand, $\mathcal{R}\left(\Lambda_{\min }\right)$ slightly decreases (zoom not shown), reaches a minimum value 21.735 at $N=0.0785(784)$ and increases. For $N \rightarrow N_{\max }=0.1764$, we find that $\mathcal{R}\left(\Lambda_{*}\right)=27.5$. Close to the maximum particle number, we have the scaling laws

$$
\begin{aligned}
\mathcal{R}\left(\Lambda_{c}\right)-\mathcal{R}\left(\Lambda_{*}\right) & \sim 106\left(N_{\max }-N\right)^{1 / 2}, \\
\mathcal{R}\left(\Lambda_{\min }\right)-\mathcal{R}\left(\Lambda_{*}\right) & \sim-29\left(N_{\max }-N\right)^{1 / 2} .
\end{aligned}
$$

We note that the prefactors are different on the two branches.

In Fig. 27 we have plotted the evolution of the energy density contrast $\mathcal{R}=\epsilon(0) / \epsilon(R)$ at the critical inverse temperatures $\eta_{c}$ and $\eta_{\min }$ as a function of $N$. For $N \rightarrow 0$, we find that $\mathcal{R}\left(\eta_{c}\right)$ tends to the nonrelativistic value 32.1 [21] (see Sec. III A). For $N \rightarrow 0, \mathcal{R}\left(\eta_{\min }\right)$ tends to the ultrarelativistic value 10.3 [1] which is different from the value 1.91 obtained in Ref. 163 for the self-gravitating black-body radiation (see Secs. III B and 


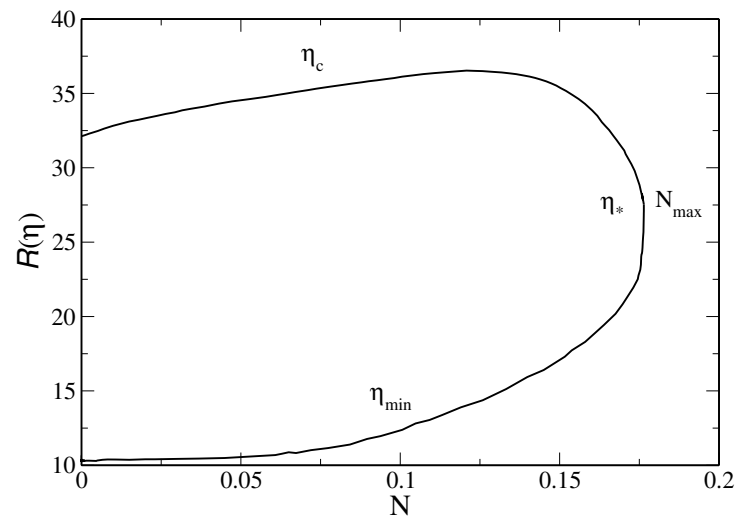

FIG. 27: Energy density contrast at the canonical collapse temperatures $\eta_{c}$ and $\eta_{\text {min }}$ as a function of $N$.

VIB). As $N$ increases, $\mathcal{R}\left(\eta_{c}\right)$ increases, reaches a maximum value 36.532 at $N=0.121(471)$ and decreases. On the other hand, $\mathcal{R}\left(\eta_{\min }\right)$ increases monotonically with $N$. For $N \rightarrow N_{\max }=0.1764$, we find that $\mathcal{R}\left(\eta_{*}\right)=27.5$. Close to the maximum particle number, we have the scaling laws

$$
\begin{array}{r}
\mathcal{R}\left(\eta_{c}\right)-\mathcal{R}\left(\eta_{*}\right) \sim 39\left(N_{\max }-N\right)^{1 / 2}, \\
\mathcal{R}\left(\eta_{\min }\right)-\mathcal{R}\left(\eta_{*}\right) \sim-113\left(N_{\max }-N\right)^{1 / 2} .
\end{array}
$$

We note that there is a sort of antisymmetry in Figs. 26 and 27 between the critical energies and critical temperatures on the cold and hot spirals respectively (see in this connection the Remark at the end of Appendix C 4 a). In particular, $\mathcal{R}\left(\Lambda_{c}\right)$ and $\mathcal{R}\left(\eta_{\min }\right)$ are monotonic functions of $N$ while $\mathcal{R}\left(\Lambda_{\text {min }}\right)$ and $\mathcal{R}\left(\eta_{c}\right)$ present an extremum.

Finally, we have plotted in Fig. 28 the minimum value of the energy density contrast $\mathcal{R}_{\text {min }}$ (see Sec. IV B as a function of $N$. For $N \rightarrow 0$, we find that $\mathcal{R}_{\min } \rightarrow 1$. For $N \rightarrow N_{\max }=0.1764$, we find that $\mathcal{R}_{\min } \rightarrow 27.5$. We also find that $\alpha\left(\mathcal{R}_{\text {min }}\right) \simeq 6.527+\ln N, \Lambda\left(\mathcal{R}_{\text {min }}\right) \sim-0.43 / N$ and $\eta\left(\mathcal{R}_{\text {min }}\right) \sim 4.33 N$ when $N \rightarrow 0$. The locus of the minimum energy density contrast is pushed to infinity when $N \rightarrow 0$.

\section{Temperature contrast}

In Fig. 29 we have plotted the evolution of the temperature contrast $\Theta=T(0) / T(R)$ at the critical energies $\Lambda_{c}$ and $\Lambda_{\min }$ as a function of $N$. The corresponding central redshift $z_{0}=e^{-\nu(0) / 2}-1$ is given by

$$
z_{0}=\frac{\Theta}{\sqrt{1-2 \mathcal{M}}}-1,
$$

where we have used the Tolman relation $T(r)=$ $T_{\infty} e^{-\nu(r) / 2}$ and Eq. (12). For $N \rightarrow 0$, we find that

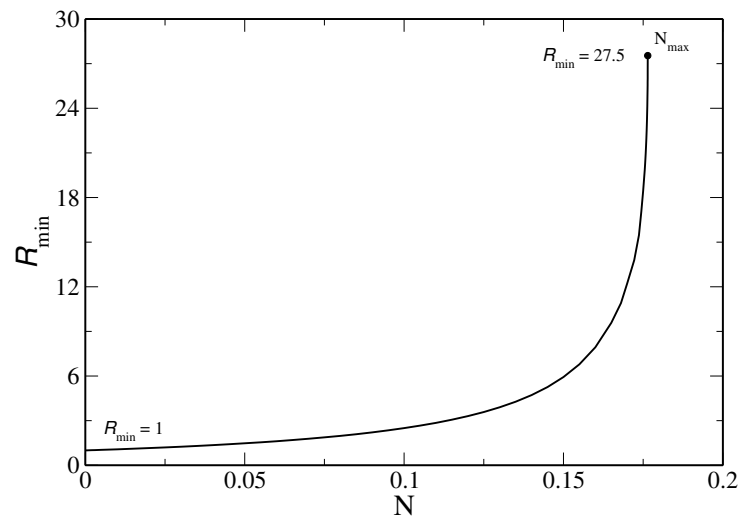

FIG. 28: Minimum value of the energy density contrast $\mathcal{R}$ as a function of $N$.

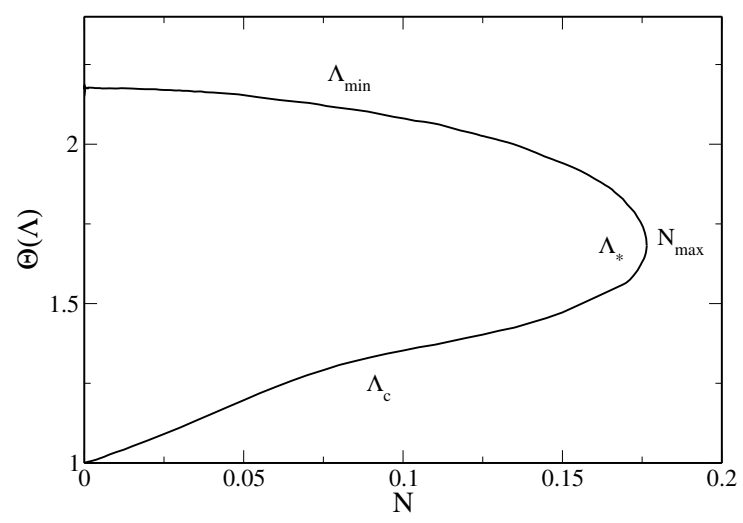

FIG. 29: Temperature contrast at the microcanonical collapse energies $\Lambda_{c}$ and $\Lambda_{\min }$ as a function of $N$.

$\Theta\left(\Lambda_{c}\right) \rightarrow 1$ since the temperature is uniform in a nonrelativistic self-gravitating system at statistical equilibrium while $\Theta\left(\Lambda_{\min }\right) \rightarrow \mathcal{R}\left(\Lambda_{\min }\right)^{1 / 4}=2.18$ for an ultrarelativistic gas (see Eq. (153) of [1), corresponding to a central redshift $z_{0}=2.06$. These results also apply to the self-gravitating black-body radiation. As $N$ increases, $\Theta\left(\Lambda_{c}\right)$ increases monotonically while $\Theta\left(\Lambda_{\min }\right)$ decreases monotonically. For $N \rightarrow N_{\max }=0.1764$, we find that $\Theta\left(\Lambda_{*}\right)=1.68$, corresponding to a central redshift $z_{0}=1.19$. Close to the maximum particle number, we have the scaling laws

$$
\begin{gathered}
\Theta\left(\Lambda_{c}\right)-\Theta\left(\Lambda_{*}\right) \sim-1.37\left(N_{\max }-N\right)^{1 / 2}, \\
\Theta\left(\Lambda_{\min }\right)-\Theta\left(\Lambda_{*}\right) \sim 1.64\left(N_{\max }-N\right)^{1 / 2} .
\end{gathered}
$$

In Fig. 30 we have plotted the evolution of the temperature contrast $\Theta=T(0) / T(R)$ at the critical inverse temperatures $\eta_{c}$ and $\eta_{\min }$ as a function of $N$. For $N \rightarrow 0$, we find that $\Theta\left(\eta_{c}\right) \rightarrow 1$ since the temperature is uniform in a nonrelativistic self-gravitating system at statistical equilibrium while $\Theta\left(\eta_{\min }\right) \rightarrow \mathcal{R}\left(\eta_{\min }\right)^{1 / 4}=1.79$ 


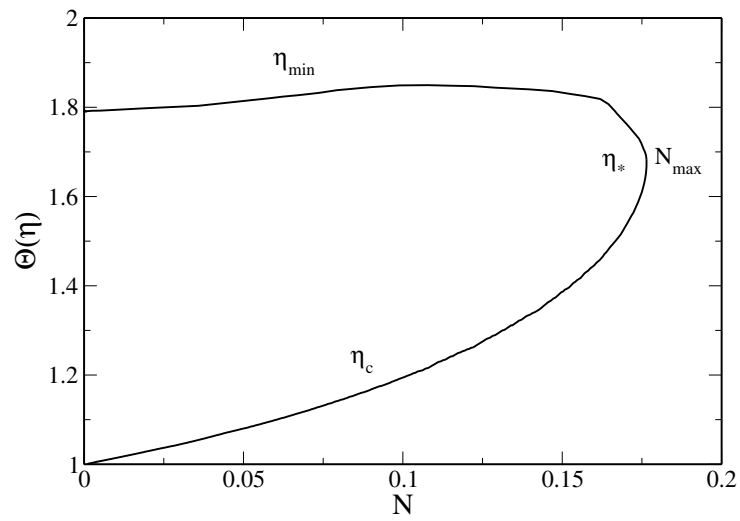

FIG. 30: Temperature contrast at the canonical collapse temperatures $\eta_{c}$ and $\eta_{\min }$ as a function of $N$.

for an ultrarelativistic gas (see Eq. (153) of [1). As $N$ increases, $\Theta\left(\eta_{c}\right)$ increases monotonically. On the other hand, $\Theta\left(\eta_{\min }\right)$ increases slightly, reaches a maximum value 1.85 at $N=0.108$ and decreases. For $N \rightarrow N_{\max }=0.1764$, we find that $\Theta\left(\eta_{*}\right)=1.68$. Close to the maximum particle number, we have the scaling laws

$$
\begin{aligned}
\Theta\left(\eta_{c}\right)-\Theta\left(\eta_{*}\right) & \sim-1.83\left(N_{\max }-N\right)^{1 / 2}, \\
\Theta\left(\eta_{\min }\right)-\Theta\left(\eta_{*}\right) & \sim 0.873\left(N_{\max }-N\right)^{1 / 2} .
\end{aligned}
$$

\section{Another normalization}

To plot the caloric curves, we have to introduce convenient normalizations of the temperature and binding energy. In the preceding sections, we have considered the normalizations $\eta$ and $\Lambda$ adapted to the nonrelativistic limit (for $\nu \rightarrow 0$ and $\Lambda, \eta \sim 1$ we are in the case $k_{B} T \ll m c^{2}$ ) and the normalizations $\mathcal{B}$ and $\mathcal{M}$ adapted to the ultrarelativistic limit (for $\nu \rightarrow 0$ and $\mathcal{M}, \mathcal{B} \sim 1$ we are in the case $\left.k_{B} T \gg m c^{2}\right)$. We could have also introduced the normalizations

$$
\frac{1}{b_{\infty}} \equiv \frac{k_{B} T_{\infty}}{m c^{2}}=\frac{\nu}{\eta}=\frac{1}{\mathcal{B} \nu}
$$

and

$$
e \equiv \frac{E}{N m c^{2}}=\frac{M}{N m}-1=-\Lambda \nu=\frac{\mathcal{M}}{\nu}-1 .
$$

Here, $e$ is called the fractional binding energy. We note that these normalized variables do not depend on the box radius $R$. Using the dimensionless variables introduced in Appendix B of [2], we get

$$
\frac{1}{b_{\infty}}=T_{\infty} \quad \text { and } \quad e=\frac{E}{N} .
$$

For $N \rightarrow 0$, the turning points of the cold and hot spirals behave with $N$ as

$$
\begin{gathered}
\frac{1}{b_{\infty}^{c}}=\frac{k_{B} T_{\infty}^{c}}{m c^{2}} \sim \frac{N}{2.52}, \\
e_{c}=\frac{E_{c}}{N m c^{2}}=\frac{M_{c}}{N m}-1 \sim-0.335 N, \\
\frac{1}{b_{\infty}^{\min }}=\frac{k_{B} T_{\infty}^{\max }}{m c^{2}} \sim \frac{1}{17.809 N}, \\
e_{\max }=\frac{E_{\max }}{N m c^{2}}=\frac{M_{\max }}{N m}-1 \sim \frac{0.24632}{N}-1 .
\end{gathered}
$$

We note that the caloric curve $b_{\infty}(e)$ does not tend to a limit when $N \rightarrow 0$. In this sense, the normalizations $\eta(\Lambda)$ and $\mathcal{B}(\mathcal{M})$ seem to be more adapted to our study than $b_{\infty}(e)$. On the other hand, for $N \rightarrow N_{\max }=0.1764$, we have

$$
\begin{gathered}
\frac{1}{b_{\infty}^{*}}=\frac{k_{B} T_{\infty}^{*}}{m c^{2}}=0.1446 \\
e_{*}=\frac{E_{*}}{N m c^{2}}=\frac{M_{*}}{N m}-1=0.1734 .
\end{gathered}
$$

The order of magnitude of $k_{B} T_{\infty}^{*} / m c^{2}=0.1446$ and the corresponding central redshift $z_{0}^{*}=1.19$ are in agreement with the maximum temperature $k_{B}\left(T_{\infty}\right)_{\max } / m c^{2}=$ 0.273 and the corresponding central redshift $z_{0}=1.08$ found by Zel'dovich and Podurets [132] for heavily truncated isothermal distributions. However, the correspondance between the two approaches is not straightforward. We refer to Appendix B of [1] for a detailed comparison between box-confined isothermal spheres and heavily truncated isothermal distributions.

\section{ASTROPHYSICAL APPLICATIONS}

The caloric curve of the general relativistic classical gas with its double spiral shape displays different types of instabilities. We discuss here some astrophysical applications of these instabilities.

\section{A. Instability at $T_{c}$ for gaseous stars and self-gravitating Brownian particles}

Let us first consider the instability that takes place at $T_{c}$ in the CE. The CE is appropriate to study a system in contact with a heat bath fixing the temperature $T$. This could be an isothermal self-gravitating gas (star) described by the Euler-Poisson equations [195, 196] or a system of self-gravitating Brownian particles described by the Smoluchowski-Poisson equations [91 97]. 
In that case, the temperature is constant and uniform. The dynamical evolution of the system is due to a departure from hydrostatic equilibrium. When $T>T_{c}$ the system settles on a stable equilibrium state in which the pressure gradient equilibrates the gravitational attraction. At $T=T_{c}$ the equilibrium becomes unstable and the system undergoes an isothermal collapse [89]. The system collapses because it is too cold so the thermal pressure cannot balance the gravitational attraction. The thermodynamical stability in the CE coincides with the dynamical stability with respect to the Euler-Poisson equations [89] and with respect to the SmoluchowskiPoisson equations [91, 92]. This leads to a fast instability at $T_{c} \cdot{ }^{43}$

The perturbation $\delta \rho(r)$ that triggers the instability at the critical temperature $T_{c}$ has a core structure (one node) [89, 196] while the velocity perturbation $\delta v(r)$ has an implosive structure (no node) with $\delta v<0$ (implosion) [89, 196. In view of the form of the marginal mode, the isothermal collapse of a star corresponds to a pure implosion (the star collapses as a whole) leading to a Dirac peak containing all the mass [93, 97, 195, 196]. This structure has an infinite free energy $(F \rightarrow-\infty)$ [92]. In practice, the collapse stops when quantum mechanics (Pauli's exclusion principle for fermions) comes into play. This results in the formation of a compact object such as a white dwarf or a neutron star containing most of the mass + a tenuous atmosphere [2, 101, 197]. This is possible as long as the mass of the star is not too high, i.e., below the Oppenheimer-Volkoff (OV) limit $M_{\mathrm{OV}}$. When $M \gtrsim M_{\mathrm{OV}}$ general relativity must be taken into account and there is no equilibrium state anymore. In that case, the star collapses towards a black hole.

This phase transition is reminiscent of the hypernova phenomenon for supermassive stars (above $40 M_{\odot}$ ) which shows very intense and directive gamma ray bursts, but no explosion of matter (or a very faint one) [196].

\section{B. Instability at $E_{c}$ for globular clusters and dark matter halos}

We now consider the instability that takes place at $E_{c}$ in the MCE. The MCE is appropriate to study an isolated system evolving at fixed energy $E$. We consider here a stellar system such as a globular cluster described by the Vlasov-Landau-Poisson equation or by the orbitaveraged-Fokker-Planck equation [120.

In that case, the system is in hydrostatic equilibrium but the temperature (velocity dispersion) is not uniform. The dynamical evolution of the system is due to a temperature gradient between the core and the halo and the

43 The instability may be slow if the system is described by the Vlasov-Kramers-Poisson equations with a small friction coefficient. fact that the specific heat of the core is negative [58, 119 121. (see Appendix B). When $E>E_{c}$ the system settles on a stable equilibrium state in which the temperature in the core is equal to the temperature in the halo (the temperature is uniform). At $E=E_{c}$ the equilibrium becomes unstable and the system undergoes the gravothermal catastrophe [58. In that case, the temperature in the core increases more rapidly than the temperature in the halo. This leads to a thermal runaway and a core collapse [58, 119-121]. The thermodynamical stability in the MCE does not coincide with the dynamical stability with respect to the (collisionless) Vlasov-Poisson equations. Indeed, all isotropic distribution functions are (Vlasov) dynamically stable [124-129]. This implies that the instability at $E_{c}$ is slow (secular) since it has a thermodynamical origin (see Sec. IVF).

The perturbation $\delta \rho(r)$ that triggers the instability at the critical energy $E_{c}$ has a core-halo structure (two nodes) 79 . In view of the form of the marginal mode, a globular cluster experiencing the gravothermal catastrophe has a core-halo structure reminiscent of a red giant [58. Core collapse leads to a binary star surrounded by a hot halo [121. This structure has an infinite entropy $(S \rightarrow+\infty)$ at fixed energy 92 .

In the case of dark matter halos made of fermions (massive neutrinos) or bosons (axions), the gravothermal catastrophe can be stopped or even prevented by quantum mechanics. This leads to a fermion or boson "ball" - forming a bulge - surrounded by an extended halo (quantum core-halo structure) [198, 199. Alternatively, during the gravothermal catastrophe, the system may become relativistic and finally undergo a dynamical instability of general relativistic origin leading to the formation of a supermassive black hole as described in 132, 151, 161. The application of this scenario to dark matter halos has been developed in 200. and advocated in [114, 197, 198].

\section{Instability at $E_{c}$ for gaseous stars and self-gravitating Brownian particles}

As before we consider the instability at $E_{c}$ in the MCE but we focus on a different dynamical model in which the temperature $T(t)$ is uniform throughout the system but evolves with time so as to conserve the energy $E$. Specifically, we consider a self-gravitating gas (star) described by the Euler-Poisson equations [201, 202] or a system of self-gravitating Brownian particles described by the Smoluchowski-Poisson equations [91, 92] with an additional equation assuring the conservation of energy.

In this model the temperature $T(t)$ is uniform (albeit not constant in time) and the dynamical evolution of the system is due to a departure from hydrostatic equilib- 
rium. ${ }^{44}$ When $E>E_{c}$ the system settles on a stable equilibrium state in which the pressure gradient equilibrates the gravitational attraction. At $E=E_{c}$, the equilibrium becomes unstable and the system undergoes a form of gravothermal catastrophe [91, 92, 201, 202]. The system collapses because it is too cold (even if the temperature increases with time) so the thermal pressure cannot balance the gravitational attraction. The thermodynamical stability in the MCE coincides with the dynamical stability with respect to the Euler-Poisson equations and with respect to the Smoluchowski-Poisson equations with a time-dependent temperature $T(t)$ [91, 92, 201, 202]. This leads to a fast (dynamical) instability at $E_{c}$ in contrast to the slow (secular) instability of globular clusters experiencing the standard gravothermal catastrophe.

The perturbation $\delta \rho(r)$ that triggers the instability at the critical energy $E_{c}$ has a core-halo structure (two nodes) [79, 92, 202] while the velocity perturbation $\delta v(r)$ has an implosive-explosive structure (one node) with $\delta v<0$ in the core (implosion) and $\delta v>0$ in the halo (explosion) 202. In view of the form of the marginal mode, the gravothermal catastrophe of a star (in the sense explained above) corresponds to an implosion-explosion leading to a collapsing core and an explosive (hot) atmosphere expanding at large distances 202]. This structure has an infinite entropy $(S \rightarrow+\infty)$ at fixed energy 92 . In practice, the collapse stops when quantum mechanics (Pauli's exclusion principle for fermions) comes into play. This results in the formation of a compact object such as a white dwarf or a neutron star containing a finite fraction $(\sim 1 / 4)$ of the total initial mass + an extended halo [2, 101, 197. This is possible as long as the mass of the star is not too high. For $M \gtrsim 4 M_{\mathrm{OV}}$ the mass of the core passes above the OV limit and there is no equilibrium state anymore. In that case, the core collapses towards a black hole.

This phase transition is reminiscent of the red giant structure of stars with low or intermediate mass (roughly $\left.0.3-8 M_{\odot}\right)$ in a late phase of stellar evolution before the white dwarf stage. The implosion of the core and the explosion of the halo is also similar to the supernova explosion of massive stars with mass in the range of $8-$ $40 M_{\odot}$ resulting in the formation of a neutron star [202].

\section{Instability at $E_{\max }$ for the self-gravitating black-body radiation}

As a preamble of the following section, let us first consider the general relativistic instability that takes place

44 This model assumes that thermodynamical equilibrium is established on a timescale short with respect to the dynamical time. By contrast, for globular clusters, thermodynamical equilibrium is established on a timescale long with respect to the dynamical time. This model also assumes an infinite thermal conductivity so that the temperature is uniform throughout the system. at $E_{\max }$ in the MCE for the self-gravitating black-body radiation [162, 163]. When $E<E_{\max }$ the system settles on a stable equilibrium state. At $E=E_{\max }$ the equilibrium becomes unstable and the system collapses because it is too hot and feels "the weight of heat" (Tolman's effect) [164. Indeed, energy is mass so that it gravitates. The thermodynamical stability in the $\mathrm{MCE}$ coincides with the dynamical stability with respect to the Euler-Einstein equations 162, 163. As a result, this is a fast instability.

The perturbation $\delta \epsilon(r)$ that triggers the instability at the critical energy $E_{\max }$ has a core structure (one node) [166. In view of the form of the marginal mode, the collapse of the self-gravitating black-body radiation at $E=E_{\max }$ corresponds to a pure implosion (the photon star collapses as a whole) leading to a black hole containing all the mass.

Remark: We note that the self-gravitating black-body radiation in the MCE behaves similarly to a nonrelativistic self-gravitating classical isothermal gas in the $\mathrm{CE}$ and differently from a nonrelativistic self-gravitating classical isothermal gas in the MCE. Indeed, for the selfgravitating black-body radiation in the MCE: (i) the thermodynamical stability coincides with the dynamical stability with respect to the Euler-Einstein equations governing the evolution of a gas with a linear equation of state $P=\epsilon / 3$; (ii) the marginal mode $\delta \epsilon(r)$ at $E_{\max }$ has a core structure; (iii) the instability is fast (dynamical). Similarly, for a nonrelativistic self-gravitating classical isothermal gas in the CE: (i) the thermodynamical stability coincides with the dynamical stability with respect to the Euler-Poisson equations governing the evolution of a gas with a linear equation of state $P=\rho k_{B} T / \mathrm{m}$; (ii) the marginal mode $\delta \rho(r)$ at $T_{c}$ has a core structure; (iii) the instability is fast (dynamical). By contrast, for a nonrelativistic self-gravitating classical isothermal gas in the MCE: (i) the thermodynamical stability does not coincide with the dynamical stability with respect to the Vlasov-Poisson equations; (ii) the marginal mode $\delta \rho(r)$ at $E_{c}$ has a core-halo structure; (iii) the instability is slow/secular (thermodynamical).

\section{E. Instability at $E_{\max }$ for a relativistic classical isothermal gas}

We finally consider the general relativistic instability that takes place at $E_{\max }$ in the MCE for a self-gravitating classical isothermal gas. The MCE is the relevant ensemble to describe relativistic star clusters that are isolated. However, the consideration of very high (positive) energies is possible only if we confine the system within a cavity. This is a very artificial situation. ${ }^{45}$ When

45 The CE may be appropriate to describe relativistic stars in contact with a heat bath. In that case, the box may be less artificial 
$E<E_{\max }$ the system settles on a stable equilibrium state. At $E=E_{\max }$ the equilibrium becomes unstable and the system collapses. The thermodynamical stability in the MCE coincides with the dynamical stability with respect to the Vlasov-Einstein equations [155]. As a result, this is a fast (dynamical) instability. This is different from the case of nonrelativistic systems experiencing the slow (thermodynamical) gravothermal catastrophe at $E_{c}$ (see Sec. IV F).

The perturbation $\delta \epsilon(r)$ that triggers the instability at the critical energy $E_{\max }$ has not been determined. By analogy with the self-gravitating black-body radiation, we suspect that it has a core structure. If confirmed, the collapse of a relativistic classical isothermal gas at $E=$ $E_{\max }$ would correspond to a pure implosion (the system collapses as a whole) leading to a black hole containing all the mass. Quantum mechanics cannot stop the collapse in this highly relativisitic situation [2, 169].

Remark: owing to the analogy between a strongly relativistic classical isothermal gas and the self-gravitating black-body radiation, we understand why thermodynamical stability coincides with dynamical stability in general relativity in the MCE similarly to Newtonian systems in the $\mathrm{CE}$ and differently from Newtonian systems in the MCE. We also understand why the marginal mode of strongly relativistic systems at $E_{\max }$ in the MCE has (presumably) a core structure similarly to the marginal mode of nonrelativistic systems at $T_{c}$ in the $\mathrm{CE}$ and differently from the marginal mode of nonrelativistic systems at $E_{c}$ in the MCE (that has a core-halo structure). The self-gravitating black-body radiation is a particular system where Ipser's conjecture [155] (see Sec. IV F) can be easily demonstrated. However, it is important to realize that, for the self-gravitating black-body radiation, dynamical stability refers to the Euler-Einstein equations while, for a classical isothermal system, it refers to the Vlasov-Einstein equations. This is a difference of fundamental importance.

\section{CONCLUSION}

In this paper, we have studied the statistical equilibrium states of box-confined classical self-gravitating systems in general relativity and we have determined their caloric curves. This is a generalization of the problem introduced by Antonov [57] and Lynden-Bell and Wood [58 for nonrelativistic classical stellar systems. This also corresponds to the nondegenerate limit of the selfgravitating Fermi gas studied by Hertel and Thirring [203, Bilic and Viollier [204] and Chavanis 101, 205] in the nonrelativistic limit and by Bilic and Viollier [168, Alberti and Chavanis 22 and Roupas and Chavanis [169. in general relativity.

as it can mimic the pressure exerted by an external medium.
The caloric curves of the general relativistic classical self-gravitating gas depend on the compactness parameter $\nu=G N m / R c^{2}$. They typically have the form of a double spiral. The system undergoes a gravitational collapse at both low and high energies and temperatures. At low temperatures the gas collapses because it is too cold and the thermal pressure cannot balance the gravitational attraction. At high energies the gas collapses because it is too hot and feels "the weight of heat" 164] (energy is mass so that it gravitates). The two spirals approach each other as the compactness parameter $\nu=G N m / R c^{2}$ increases, indicating that general relativistic effects advance the onset of gravitational collapse. There is a maximum compactness $\nu_{\max }=0.1764$ above which no equilibrium state exists whatever the values of energy and temperature.

As mentioned in the Introduction, similar results have been obtained by Roupas [167]. Below, we make a brief comparison between the two studies in order to show that they are complementary to each other:

(i) We have made a detailed history of the statistical mechanics of self-gravitating systems in Newtonian gravity and general relativity (see also [1-4). In particular, we have stressed the important works of Zel'dovich and Podurets [132, Ipser [140, 155] and Katz and Horwitz [152, 154] related to the general relativistic classical isothermal gas. We have discussed the analogies and the differences between the maximum temperature $k_{B}\left(T_{\infty}\right)_{\max } / m c^{2}=0.273$ (and the corresponding central redshift $z_{0}=1.08$ ) of heavily truncated isothermal distributions discovered by Zel'dovich and Podurets [132] and the maximum temperature $k_{B} T_{\infty}^{*} / m c^{2}=0.1446$ (and the corresponding central redshift $z_{0}^{*}=1.19$ ) of box-confined isothermal systems at $\nu_{\max }=0.1764$. This comparison is further developed in Appendix B of [1].

(ii) We have shown that the "hot spiral" of the caloric curve of general relativistic classical self-gravitating systems has some similarities with the caloric curve of the self-gravitating black-body radiation represented in Fig 15 of 163 that also displays a striking spiral structure. In particular, the values of the maximum mass-energy $G M_{\max } / R c^{2}=0.24632$ (and the corresponding density contrast $\mathcal{R}_{\mathrm{MCE}}=22.4$ ) are the same. This is no true, however, for the maximum temperature because the two systems have a different physical nature. This comparison is further developed in Sec. VI of [1].

(iii) We have used a different numerical method to obtain the caloric curves of the general relativistic classical gas which is based on the algorithm proposed by Bilic and Viollier 168 for self-gravitating fermions. This method (described in Appendix C) allows us to understand clearly the origin of the critical parameters $N_{S}^{\prime}, N_{S}$, $N_{\max }, \eta_{c}, \Lambda_{c}, \eta_{\min }, \Lambda_{\min } \ldots$ appearing in our study and to determine them from simple graphical constructions.

(iv) We have shown that the curves $\Lambda(\mathcal{R})$ and $\eta(\mathcal{R})$ close themselves at high density contrasts and that they contain a "relevant" part and an "irrelevant" part. The "irrelevant" part (corresponding to unstable equilibrium 
states with a very high central density) may be of interest to mathematicians in order to understand better the rich and complex structure of the isothermal TOV equations presented in Sec. III.

(v) We have plotted the curves $S(E)$ and $F(T)$ (see Figs. 9 and 10 and explained why they display spikes at the critical points.

(vi) We have discussed the thermodynamical and dynamical stability of isothermal star clusters in general relativity (see Sec. IVF). We have argued that the instability that occurs at low energies (cold spiral) is slow/secular, like in the case of globular clusters experiencing the gravothermal catastrophe, because it has a thermodynamical origin while the instability that occurs at high energies (hot spiral) is fast because it has a dynamical origin. ${ }^{46}$ This is corroborated by the fact that the marginal mode of instability at $E_{\max }$ (hot spiral - strongly relativistic configurations) presumably has a "core" structure, like for the self-gravitating black-body radiation [163, 166], contrary to the marginal mode of instability at $E_{c}$ (cold spiral - weakly relativistic configurations) that has a "core-halo" structure [79] (see the discussion Sec. VIII).

(vii) We have made a detailed study of the caloric curve close to $N_{\max }=0.1764$ showing that it has a complex structure giving rise to amputed spirals, and a loop, before disappearing (see Figs. 12 and 13). This complex topological structure may be of interest to mathematicians. Note that the stable part of the caloric curve remains however "simple".

(viii) We have compared the caloric curves of general relativistic classical systems for $N \rightarrow 0$ (see Fig. 16) with the caloric curves obtained in the case of Newtonian selfgravitating classical systems described by a relativistic equation of state (see Fig. 1 of [166]). We have shown that the critical energy $E_{c}$ increases with $N$ in the two cases. By contrast, the critical temperature $T_{c}$ increases with $N$ for general relativistic systems while it remains constant for relativistic Newtonian systems [166. On the other hand, the hot spiral is absent for Newtonian systems.

(ix) Following [1, we have introduced a new normalization of the energy and temperature, $\mathcal{M}=G M / R c^{2}$ and $\mathcal{B}=R c^{4} / G N k_{B} T_{\infty}$, adapted to the ultrarelativistic

${ }^{46}$ If we extrapolate the conjecture of Ipser 155 to box-confined systems, and argue that thermodynamical and dynamical stability coincide in general relativity, we are led to conclude that general relativistic systems essentially experience a (fast) dynamical instability. As a result, the collapse at $E_{\max }$ (hot spiral - strongly relativistic configurations) occurs quickly, on a dynamical timescale. This is very different from the gravothermal catastrophe of nonrelativistic systems which is a (slow) thermodynamical instability. As a result, the collapse at $E_{c}$ (cold spiral - weakly relativistic configurations) occurs secularly, on a relaxation timescale of the order of the age of the Universe. In this sense, the collapse at high energies is very different from the collapse at low energies. limit. Using this normalization, the caloric curve $\mathcal{B}(\mathcal{M})$ tends to an asymptotic curve when $\nu \rightarrow 0$ (see Figs. 19 and 20). This asymptotic curve (hot spiral) has been theoretically characterized in [1]. We have compared this caloric curve, corresponding to the ultrarelativistic limit of the classical self-gravitating gas, with the caloric curve of the self-gravitating black-body radiation (see Fig. 22. This comparison is further developed in Sec. VI of [1. ${ }^{47}$

(x) We have performed a detailed study of the evolution of the critical points of the general relativistic classical gas with $N$ obtaining their asymptotic behaviors when $N \rightarrow 0$ and $N \rightarrow N_{\max }$.

(xi) We have discussed (see also [2, 170, 202]) some analogies between the implosion-explosion instability that occurs at low energies (cold spiral) in certain models of stars 202 and the supernova phenomenon. A different scenario of supernova explosion connected to the gravitational collapse occurring at very high positive energies (hot spiral) is developed by Roupas [206, 207.

\section{Appendix A: The very early history of the theory of globular clusters}

In this Appendix, we briefly retrace the very early history of the theory of globular clusters. This completes the review of the statistical mechanics of self-gravitating systems made in the introduction.

Inspired by an idea of Lord Kelvin, von Zeipel 208. modeled the density distribution of stars in a globular cluster by analogy with the density profile of a selfgravitating isothermal gas and found a good agreement with observations in the central parts of the cluster. Plummer [209], on the other hand, modeled the density distribution of stars in a globular cluster by analogy with the density profile of a self-gravitating polytropic gas in convective equilibrium whose general theory had been elaborated by Lord Kelvin [210, 211, Lane [212, Ritter [213] and Emden [21]. He considered analytical polytropic models: the polytrope of index $n=1$ found by Ritter 213 and the polytrope of index $n=5$ found by Schuster [214]. He noted that the polytrope of index $n=5$, whose spatial density decreases at large distances as $r^{-5}$, provides a good agreement with observations in the outer parts of the cluster. This is now called the "Plummer distribution". At the end of his paper, Plummer proposed to model the density of stars in a globular cluster by an isothermal distribution in the center and by a polytropic distribution of index $n=5$ in the envelope. In other words, he assumed that globular clusters have a central core in isothermal equilibrium and

\footnotetext{
47 To investigate the ultrarelativistic limit, Roupas [167] uses the normalized temperature $k_{B} T_{\infty} / m c^{2}$ (see his Fig. 7(a)). However, as explained in Sec. VIID this normalization does not allow one to obtain an asymptotic caloric curve when $\nu \rightarrow 0$. This is apparent on his Fig. 7(a).
} 
an outer envelope in convective equilibrium. Eddington 23. 215] argued that globular clusters are collisionless stellar systems (instead of collisional gases as assumed by von Zeipel 208, and Plummer 209]) and derived a selfconsistent quasisteady state with a Schwarzschild velocity distribution containing the Maxwell distribution as a special case. Jeans [18] determined the general form of steady state solutions of the collisionless Boltzmann equation (deriving the so-called "Jeans theorem" 216]) and pointed out that the Maxwell-Boltzmann distribution is a particular case of his general theorem. Jeans [7] mentioned that the effect of "collisions" (encounters) between stars would tend to establish an isothermal (Maxwell-Boltzmann) distribution. However, since the density of an isothermal sphere decreases as $r^{-2}$ at large distances (implying an infinite mass) the collisions would lead to the complete disintegration of the cluster if they were allowed to act for a sufficient time. As a result, in a subsequent paper, Jeans [217] modeled globular clusters as collisionless stellar systems with a distribution function of the form $f=f(\epsilon)$ satisfying the condition $f(\epsilon)=0$ when $\epsilon>0$ (for a permanent cluster the energy of every star must be negative). He found that the density decreases at large distances as $r^{-4}$. He argued that this law provides a better description of globular clusters than the Plummer law.

Eddington 24] reconsidered the results of von Zeipel and Plummer from the point of view of collisionless stellar systems instead of collisional gases. The connection is made through the so-called "Eddington formula" 216] which relates the distribution function $f(\epsilon)$ of a spherical stellar system to the density $\rho(\Phi)$ of the corresponding barotropic gas. In particular, he introduced the stellar polytropic distributions $f=A\left(\epsilon_{m}-\epsilon\right)^{n-3 / 2}$ associated with the polytropic gaseous spheres. Eddington tried to find physical arguments to justify the Plummer distribution (polytrope $n=5$ ) but did not arrive at a justification for this particular law. Following Jeans, he insisted on the fact that the distribution function $f(\epsilon)$ must vanish for positive energies $(\epsilon \geq 0)$ in order to account for the escape of high energy stars and avoid an infinite mass. He introduced the truncated Boltzmann distribution $f=A e^{-\beta \epsilon}$ if $\epsilon \leq 0$ and $f=0$ if $\epsilon \geq 0$ that will be studied in detail later by Woolley [29. ${ }^{48}$

48 In addition, the paper of Eddington [24] contains many insight and intuitions about: (i) the escape of stars studied lated by Ambartsumian [15] and Spitzer [17]: "the system will settle down to a state which may be considered steady, since it is only slowly altered by the encounters of the stars. Stars with high velocities may be lost in the process; it is highly probable that some will escape since the condition for a steady state $T=\frac{1}{2} \Omega$, shows that on the average the mean kinetic energy of the stars is as much as a quarter of the kinetic energy required for escape."; (ii) the violent relaxation of collisionless stellar systems studied later by Lynden-Bell [56]: "The system might oscillate at first; but, since the periods of the individual stars are not isochronous, the oscillations would die out rapidly. The question arises whether
Heckmann and Siedentopf [25] treated globular clusters as collisional stellar systems described by the Boltzmann equation. By cancelling simultaneously the advection term and the collisional term in the Boltzmann equation, they derived from it the Maxwell-Boltzmann distribution function $f(\mathbf{r}, \mathbf{v})$ and the Boltzmann density distribution $\rho(\mathbf{r})$ coupled to the Poisson equation. On the other hand, Mineur [16], Dicke [26] and Spitzer [17] assumed that globular clusters have reached a state of statistical equilibrium and that they are described by the Boltzmann-Poisson equations applying to isothermal self-gravitating systems. The next developments in the study of globular clusters and in the statistical mechanics of classical self-gravitating systems are reviewed in the Introduction.

\section{Appendix B: Negative specific heats and ensembles inequivalence}

In this Appendix, we show how the concepts of negative specific heats and ensembles inequivalence for systems with long-range interactions emerged in physics and astrophysics.

The notion of negative specific heats was known to astronomers since the 19 th century. Indeed, according to the virial theorem, when a star or a star cluster loses energy its temperature increases [218, 219].

In statistical physics, Landau and Lifshitz [220] (P. 62) mentioned that inhomogeneous systems whose energy is nonadditive, such as self-gravitating systems, may have negative specific heats $C=d E / d T<0$. As a result, the body gets hotter when its energy decreases. They stressed that this property is not in contradiction with the laws of thermodynamics.

Lynden-Bell and Wood [58] proved from the virial theorem that self-gravitating systems have negative specific heat (they also showed that the presence of a box in their model does not alter this result provided the system is sufficiently centrally condensed). By losing heat they grow hotter. The evolution is thus away from equilibrium: If two systems with negative specific heat are put in contact, the hotter loses heat and gets hotter while the colder gains heat and gets colder. For the same reason, no equilibrium is possible for a system of negative specific

the ultimate steady state may not be practically independent of the initial conditions; and if so, can we show that it is the state which the actual clusters assume?"; (iii) the King 44 model: "The law of distribution should therefore be approximately the Maxwellian law for small velocities, but modified for the larger velocities so as to fall to zero at or before the limit $\sqrt{2 \Phi}$ "; (iv) the maximum entropy state and the $H$-theorem studied lated by Ogorodnikov [54, 55, Antonov [57] and Lynden-Bell and Wood [58]: "We assume then that the most probable distribution is that which leads to the lowest value of $H$, subject to the condition that the mass is given and the energy is not greater than a fixed value". 
heat in contact with a heat bath (canonical ensemble). If the system is cooler than the bath it will accept heat and grow cooler. Inversely, if the system is hotter than the bath it will lose heat and become hotter. Finally, isolated systems (microcanonical ensemble) may also experience a similar instability. If they are sufficiently concentrated, they take a core-halo structure with a temperature gradient between the core and the halo (the core being hotter than the halo). The inner parts being self-gravitating have negative specific heat $C_{c}<0$; by losing heat they shrink and grow hotter. The outer parts (which may be held by the walls of a box) have positive specific heat $C_{h}>0$. On receiving heat they grow hotter (and expand if they are not artificially confined). If $C_{h}<\left|C_{c}\right|$ the temperature increases faster in the halo than in the core and the system as a whole can reach an equilibrium state. If $C_{h}>\left|C_{c}\right|$ the temperature difference increases and no equilibrium is possible. This is the gravothermal catatrophe. According to the previous considerations, it occurs when the total specific heat $C=-\left|C_{c}\right|+C_{h}$ passes from negative to positive values (this is in accordance with the Poincaré turning point criterion). A system of negative heat capacity can only be in equilibrium with a system of positive heat capacity provided that the sum of the heat capacities is negative. Lynden-Bell and Wood [58] related the core-halo structure of a system undergoing the gravothermal catastrophe to the onset of red-giant structure in stellar evolution. They also anticipated the existence of microcanonical phase transitions in a self-gravitating gas of fermions or hard spheres (see their Appendix IV).

Thirring 221 mentioned that stars have negative specific heat $C=d E / d T<0$. They become hotter when energy is lost. However, a body with negative specific heat cannot exist in thermal balance with a heat bath. It undergoes a first order phase transition to a new state where the body has a positive specific heat. This happens, for example, for sufficiently massive white dwarfs (above the Chandrasekhar 222] limit) when the zeropoint pressure of the electrons are no longer able to counterbalance the gravitational attraction. This leads to one of those cosmic catastrophes which we see as supernovae. The result is a pulsar (rotating neutron star) surrounded by a cloud like the crab nebula. Thirring [221] mentioned that a sufficiently massive neutron star (above the Oppenheimer-Volkoff [165] limit) may itself contract to a "mathematical point" but considered this possibility as "science fiction". This "mathematical point" is now interpreted as a black hole.

In a subsequent paper, Thirring 72 refined his arguments. Referring to Landau and Lifshitz 220] and ter Haar 223], he mentioned that self-gravitating systems may have negative specific heat for some energies. In contact with a heat bath a system with $C<0$ creates an instability leading to a phase transition. He argued that supernovae are, in essence, a phase transition of this origin. He showed from the virial theorem that $C=-(3 / 2) N k_{B}<0$ in the microcanonical ensemble but recalled that, in the canonical ensemble, the specific heat is necessarily positive. ${ }^{49}$ Therefore, the statistical ensembles are not equivalent. ${ }^{50} \mathrm{He}$ argued that the region $C<0$ is jumped by a phase transition of the first kind. He argued that stars act like systems with $C<0$. At the end of their life, when no more nuclear fuel is available, the star takes a core-halo structure similar to a red giant or a supernova. These events reflect the instability of systems with negative specific heat. Similarly, stellar systems may separate into a collapsing core and a halo. However, the timescale governing these phase transitions is very different in the two cases. For supernovae where the energy is carried quickly by neutrinos they are fast (a few days), but for stellar systems they are very slow (of the order of the age of the Universe).

In later years, Carlitz 227] showed that negative specific heat and ensembles inequivalence also occur in hadronic matter at high density. On the other hand, Bekenstein [228] and Hawking [229, 230] showed that the thermodynamics of black holes involves negative specific heats. The negative specific heat paradox was further discussed by Lynden-Bell and Lynden-Bell [231] and Padmanabhan [79] with the aid of analytical toy models. Some reviews about negative specific heats and ensembles inequivalence in self-gravitating systems are provided by Padmanabhan 79, Lynden-Bell 232, Katz 99. and Chavanis [101. More general results valid for arbitrary systems with long-range interactions are given in [185] 187.

49 In the microcanonical ensemble, using the virial theorem $2 K+$ $W=0$ with $K=(3 / 2) N k_{B} T$ we get $E=K+W=-K=$ $-(3 / 2) N k_{B} T$ implying $C=d E / d T=-(3 / 2) N k_{B}<0$. In the canonical ensemble, using the partition function, one can show that $C=d\langle E\rangle / d T=\beta^{2}\left(\left\langle E^{2}\right\rangle-\langle E\rangle^{2}\right)>0$ so the specific heat is a measure of the variance of the fluctuations of energy. In general, equivalence of the canonical and microcanonical descriptions is obtained whenever fluctuations of energy are small. The canonical and microcanonical descriptions will be inequivalent if the fluctuations are large $\left(\left\langle E^{2}\right\rangle-\langle E\rangle^{2}\right) /\left\langle E^{2}\right\rangle \gg 1$ that is to say when $C \rightarrow+\infty$ corresponding to the turning point of temperature on the caloric curve.

50 To illustrate these properties, Thirring [72] considered the statistical mechanics of one particle in a Coulombian potential. This can be seen as a preamble to the binary star model developed later by Padmanabhan [79. On the other hand, Thirring 72 and Hertel and Thirring 224] developped an analytical toy model which presents two phases, one gaseous, the other consisting of gas and one cluster of condensed matter. In the microcanonical ensemble, there is a region of negative specific heat and a first order phase transition marked by a jump of temperature at some energy. In the canonical ensemble the region of negative specific heat is replaced by a first order phase transition marked by a jump of energy at some temperature. This is similar to the phase transitions in a self-gravitating gas of fermions [203] (see a detailed history in 2, 101]) or hard spheres 225]. This is also similar to phase transitions in the van der Waals gas [226]. 


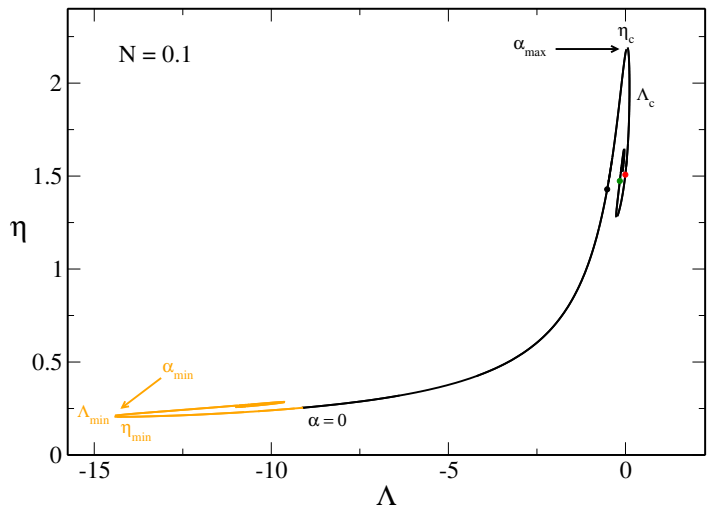

FIG. 31: Caloric curve $\eta(\Lambda)$ for $N=0.1$. The black part corresponds to $\alpha>0$ (positive chemical potential) and the orange part corresponds to $\alpha<0$ (negative chemical potential).

\section{Appendix C: Construction of the caloric curves}

In order to obtain the caloric curves $\eta(\Lambda)$ of a classical self-gravitating gas in general relativity, we have followed the method of Bilic and Viollier [168].

\section{The principle of the method}

Let us recall the procedure and illustrate it with a simple example. To construct the caloric curve $\eta(\Lambda)$ corresponding to $N=0.1$ (see Fig. 31), we proceeed as follows. We first fix a value of $\alpha$. For this value of $\alpha$, we can solve the TOV equations $(7)$ and (8) by prescribing the value $\Phi_{0}$ of the potential at the origin [we work in terms of $\Phi(r)$ instead of $b(r)$ using Eq. [16]]. We stop the integration at $r=R=1$ and compute the number of particles $N$, the mass $M$ and the Tolman temperature $T_{\infty}$ from Eqs. (10)-(12). Using Eq. (17) we obtain $\Lambda$ and $\eta$. We then vary $\Phi_{0}$ from -1 to $+\infty$ and plot $N$ as a function of $\Phi_{0}$ for the initially fixed value of $\alpha$. An example of curve $N_{\alpha}\left(\Phi_{0}\right)$ is shown in Fig. 32. This curve displays damped oscillations for large values of $\Phi_{0} .{ }^{51}$

We now consider the possible intersection(s) between the curve $N_{\alpha}\left(\Phi_{0}\right)$ and the line level $N$. Depending on the value of $N$, there can be zero, one, or several (up

${ }^{51}$ For very large values of $\Phi_{0}$ the oscillations are revived and the curve at high fields mirrors the curve at low fields. This is, however, essentially a mathematical curiosity because the solutions associated to these revived oscillations are unstable. They lead to the right parts of the curves of Figs. 4 and 5 that are unstable (see footnote 35 ). Therefore, we shall not pay too much attention on this part of the curve that we call "irrelevant". In the following, we shall focus essentially on the "relevant" part of the curve corresponding to "low" values of $\Phi_{0}$, up to the end of the damped oscillations.

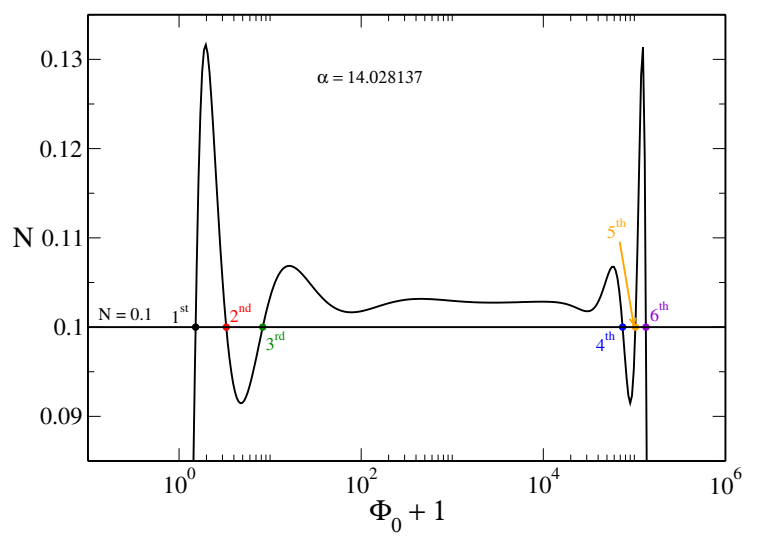

FIG. 32: The curve $N_{\alpha}\left(\Phi_{0}\right)$ for a fixed value of $\alpha$ (here $\alpha=$ 14.028137) illustrating the principle of the method detailed in the text. We have selected $N=0.1$. The first three intersections correspond to equilibrium states with $\left(\Phi_{0}, \Lambda, \eta\right)_{1}=$ $(0.5207,-0.5164,1.4286), \quad\left(\Phi_{0}, \Lambda, \eta\right)_{2}=(2.3008,-3.663 \times$ $\left.10^{-3}, 1.5076\right)$ and $\left(\Phi_{0}, \Lambda, \eta\right)_{3}=(7.3043,-0.1595,1.4736)$. They have been localized on the caloric curve of Fig. 31 by using the same color convention (black, red, green). The subsequent intersections are "irrelevant" as explained in footnote 51.

to an infinity) intersections. In the example shown in Fig. 32, corresponding to $\alpha=14.028137$ and $N=0.1$, there are three intersections in the relevant part of the curve (see footnote 51). These intersections correspond to equilibrium states that have the same values of $N$ and $\alpha$ but that are characterized by different values of $\Phi_{0}$ that we denote by $\left(\Phi_{0}\right)_{i \in\{1, \ldots, n\}}$. In the present example, $n=3$. The corresponding profiles of $\Phi(r)$ and $\epsilon(r)$ are represented in Figs. 33 and 34 for illustration. These solutions have different masses $M$ and different Tolman temperatures $T_{\infty}$, hence different values of $\Lambda$ and $\eta$. For the selected value of $N$ and for the initially fixed value of $\alpha$ we can report these solutions on the caloric curve $\eta(\Lambda)$. In our example, this defines three points (black, red and green) in Fig. 31. By varying $\alpha$ these points form $n$ branches in the caloric curve $\eta(\Lambda)$. The branches $B_{1}$ and $B_{2}$ corresponding to the first and second intersections have been represented in color (black and red) in Fig. 35.

In conclusion, the complete caloric curve $\eta(\Lambda)$ for the selected value of $N$ is obtained by determining the intersections between the curve $N_{\alpha}\left(\Phi_{0}\right)$ and the line level $N$ for "all" values of $\alpha$ ranging from $-\infty$ to $+\infty$. We can then redo this work for different values of $N$ in order to see how the caloric curve $\eta(\Lambda)$ changes with the number of particles. This leads to the caloric curves presented in Sec. V] 


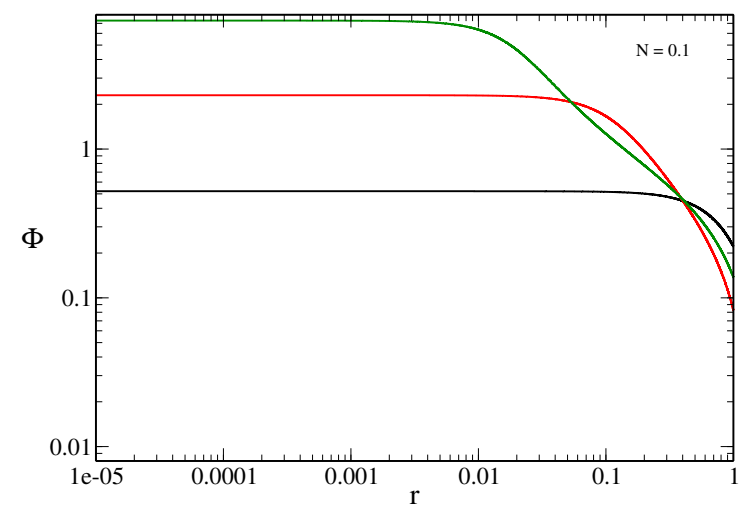

FIG. 33: Gravitational potential $\Phi(r)$ corresponding to the three intersections displayed in Fig. 32

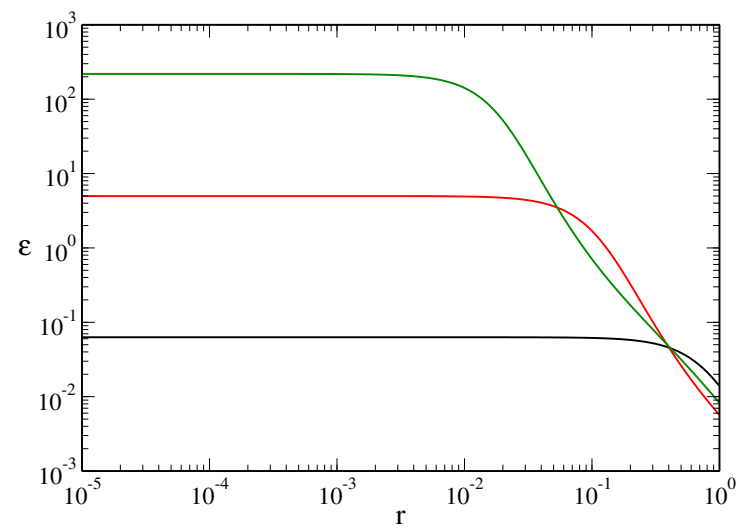

FIG. 34: Energy density profile $\epsilon(r)$ corresponding to the three intersections displayed in Fig. 32

\section{Evolution of $N_{\alpha}\left(\Phi_{0}\right)$ with $\alpha$}

We now describe how the curve $N_{\alpha}\left(\Phi_{0}\right)$ changes with $\alpha$ (as explained in footnote 51, we only consider the relevant part of the curve $N_{\alpha}\left(\Phi_{0}\right)$ ). To facilitate the discussion, we introduce some notations. We call $\mathcal{N}(\alpha)$ the maximum value of the curve $N_{\alpha}\left(\Phi_{0}\right)$ and we denote by $\Psi(\alpha)$ the value of the central potential $\Phi_{0}$ corresponding to this maximum.

We note that the curve $N_{0}\left(\Phi_{0}\right)$ corresponding to $\alpha=$ 0 is singular because $\alpha$ appears explicitly in the relation $T_{0}=\frac{1}{|\alpha|} \sqrt{\Phi_{0}+1}$ between $T_{0}$ and $\Phi_{0}$ [see Eq. [16)]. Therefore, when $\alpha \rightarrow 0$ the curves $N_{\alpha}\left(\Phi_{0}\right)$ are "squeezed" near $\Phi_{0}=-1$. This is, however, just an apparent singularity that would have not occurred if we had chosen to plot $N_{\alpha}$ as a function of $b_{0}$ instead of $\Phi_{0}$.

Let us first consider the case $\alpha<0$ (see Fig. 37). When $\alpha \rightarrow-\infty$, we find that $\mathcal{N}(\alpha) \rightarrow 0$ and $\Psi(\alpha) \rightarrow+\infty$. This corresponds to the ultrarelativistic regime that gives rise to the hot spiral in the limit $N \rightarrow 0$ (see Sec. VIB). As $\alpha$ increases, $\mathcal{N}(\alpha)$ increases and $\Psi(\alpha)$ decreases: the peak of the curve $N_{\alpha}\left(\Phi_{0}\right)$ grows and moves to the left.

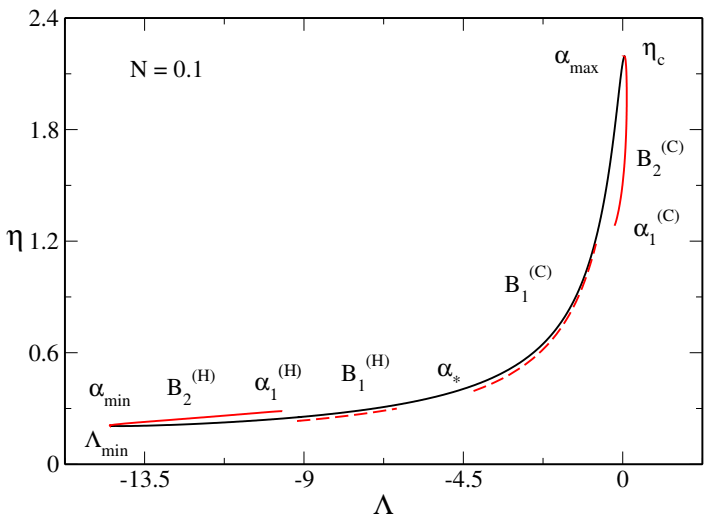

FIG. 35: Caloric curve $\eta(\Lambda)$ for $N=0.1$ showing just the branches $B_{1}$ and $B_{2}$ corresponding to the first two intersections in Fig. 32 (extended to all values of $\alpha \in\left[\alpha_{\min }, \alpha_{\max }\right]$ ). The branches $B_{1}^{(H)}$ and $B_{1}^{(C)}$ (black) correspond to the ensemble of the first intersections. They give rise to the main branch. The branch $B_{1}^{(H)}$ corresponds to $\alpha_{\min } \leq \alpha \leq \alpha_{*}$ and the branch $B_{1}^{(C)}$ corresponds to $\alpha_{*} \leq \alpha \leq \alpha_{\max }$. They connect each other at $\alpha=\alpha_{*}$. The branches $B_{2}^{(H)}$ and $B_{2}^{(C)}$ (red) correspond to the ensemble of the second intersections. They give rise to the begining of the hot $(\mathrm{H})$ and cold $(\mathrm{C})$ spirals. The branch $B_{2}^{(H)}$ corresponds to $\alpha_{\min } \leq \alpha \leq \alpha_{1}^{(H)}$. It connects the branch $B_{1}^{(H)}$ at $\alpha_{\text {min }}$ and stops at $\alpha_{1}^{(\bar{H})}$. The branch $B_{2}^{(C)}$ corresponds to $\alpha_{1}^{(C)} \leq \alpha \leq \alpha_{\max }$. It starts at $\alpha_{1}^{(C)}$ and connects the branch $B_{1}^{(C)}$ at $\alpha_{\max }$. Next order intersections (not represented) form the other branches of the spirals. We observe that a part of the second branches $B_{2}$ is superimposed on the main branch $B_{1}$ (we have slightly shifted the red curves lying on the black curve for a better visualization). They correspond to different equilibrium states with the same energy and the same temperature but a different density contrast (see the right parts in red of Figs. 4 and 5 . These solutions which have a very high central density (see Fig. 36) are irrelevant because they are unstable (see footnote 51). We note that the branch $B_{2}^{\prime}$ (dashed line) corresponds to the branch of last intersections that becomes a branch of second intersections when the branch $B_{2}$ disappears.

When $\alpha \rightarrow 0$, we find that $\mathcal{N}(\alpha) \rightarrow N_{0}=0.1297$ and $\Psi(\alpha) \rightarrow-1$ : the peak of the curve $N_{\alpha}\left(\Phi_{0}\right)$ is squeezed near $\Phi_{0}=-1$.

We now turn to the case $\alpha>0$ (see Fig. 38). When $0<\alpha \leq \alpha_{*}=5.012$, we find that $\mathcal{N}(\alpha)$ and $\Psi(\alpha)$ both increase: the peak of the curve $N_{\alpha}\left(\Phi_{0}\right)$ grows and moves to the right. When $\alpha=\alpha_{*}, \mathcal{N}(\alpha)$ reaches its maximum value $N_{\max }=0.1764$ at $\Psi_{*}=1.51157$. The first minimum and the second maximum of the curve $N_{\alpha_{*}}\left(\Phi_{0}\right)$ will play a particular role in the interpretation of the caloric curves (see below). The values of $N$ at these points are $N_{S}^{\prime}=0.128$ and $N_{S}=0.1415$. When $\alpha \geq \alpha_{*}, \mathcal{N}(\alpha)$ and $\Psi(\alpha)$ both decrease: the peak of the curve $N_{\alpha}\left(\Phi_{0}\right)$ decays and moves to the left. When $\alpha \rightarrow+\infty$, we find that $\mathcal{N}(\alpha) \rightarrow 0$ and $\Psi(\alpha) \rightarrow 0$. This corresponds to the nonrelativistic regime that gives rise to the cold spiral in 


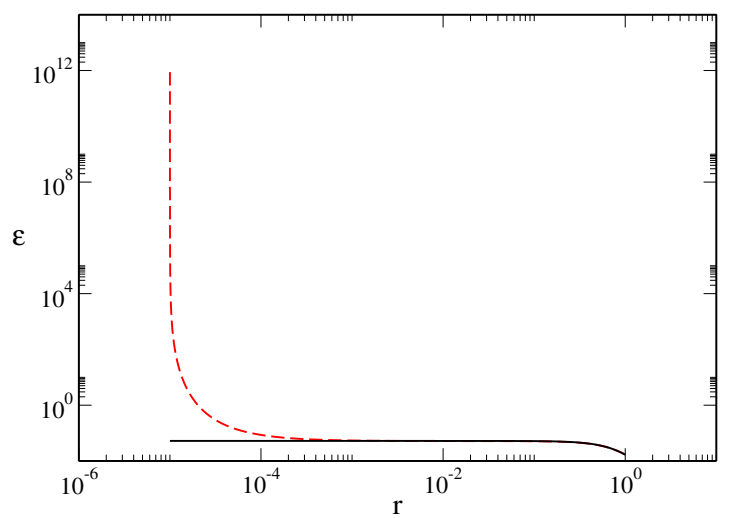

FIG. 36: Energy density profile of a stable solution (black solid line) and of an unstable solution (red dashed line) with the same value of energy and temperature (specifically $\alpha=9.77, \eta=1.01$ and $\Lambda=-1.09$ ). These solutions are superimposed on the main branch of the caloric curve (see Fig. 35. We see that their profiles coincide except at the very center where the unstable solution has a very high density.

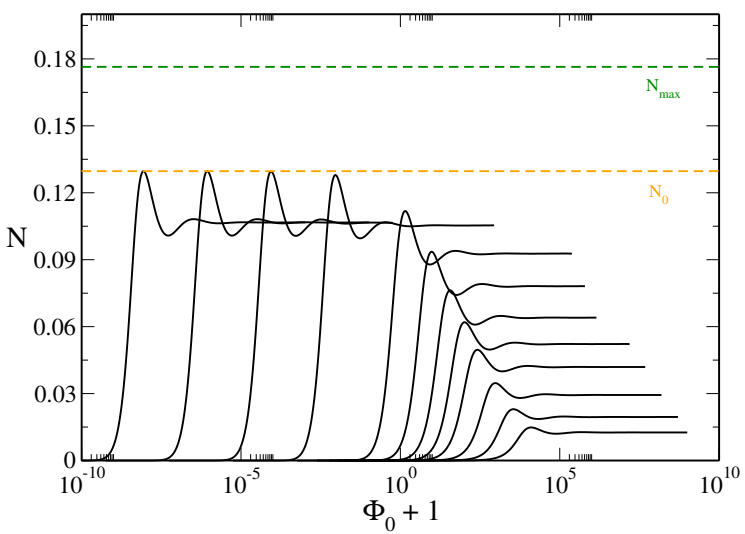

FIG. 37: Evolution of the curve $N_{\alpha}\left(\Phi_{0}\right)$ for different values of $\alpha<0$ (for illustration, the curves go from $\alpha=-10$ to $\left.\alpha=-10^{-6}\right)$. We have indicated different characteristic values of $N: N_{0}=0.1297$ and $N_{\max }=0.1764$.

the limit $N \rightarrow 0$ (see Sec. VIA).

\section{Extremal values of $\alpha$ for a given $N$}

Let us select a value of $N$ and progressively increase $\alpha$, starting from $\alpha \rightarrow-\infty$ (see Fig. 39).

For small values of $\alpha$, there is no intersection between the line level $N$ and the curve $N_{\alpha}\left(\Phi_{0}\right)$. However, as the peak $\mathcal{N}(\alpha)$ grows as $\alpha$ increases, some intersections become possible. The first intersection with the line level $N$ occurs for $\alpha=\alpha_{\min }(N)$. For $\alpha>\alpha_{\min }(N)$ the peak $\mathcal{N}(\alpha)$ continues to grow, reaches a maximum $N_{\max }=$ 0.1764 at $\alpha_{*}=5.012$, then decreases. The last intersection with the line level $N$ occurs for $\alpha=\alpha_{\max }(N)$. For

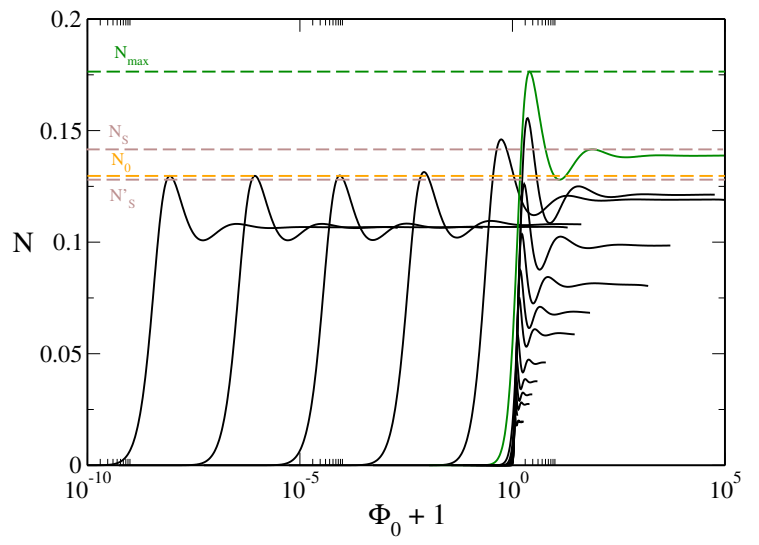

FIG. 38: Evolution of the curve $N_{\alpha}\left(\Phi_{0}\right)$ for different values of $\alpha>0$ (for illustration, the curves go from $\alpha=10^{-6}$ to $\alpha=$ 100). We have indicated different characteristic values of $N$ : $N_{0}=0.1297, N_{\max }=0.1764, N_{S}^{\prime}=0.128$ and $N_{S}=0.1415$.

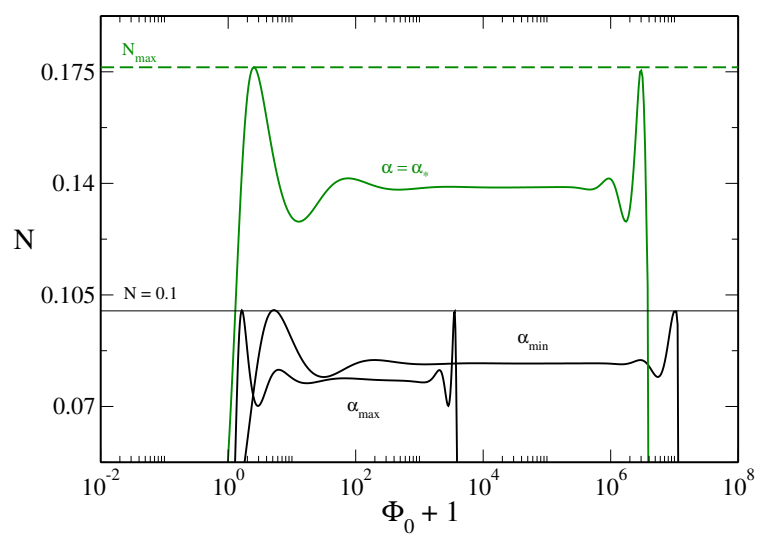

FIG. 39: Evolution of the curve $N_{\alpha}\left(\Phi_{0}\right)$ with $\alpha$ illustrating the first intersection with the line level $N$ occuring at $\alpha_{\min }(N)$ and the last intersection occuring at $\alpha_{\max }(N)$. There is no intersection below $\alpha_{\min }(N)$ or above $\alpha_{\max }(N)$. For illustration we have taken $N=0.1$ for which $\alpha_{\min }=-1.641$ and $\alpha_{\max }=20.989$. We have also represented the curve $N_{\alpha_{*}=5.012}\left(\Phi_{0}\right)$ whose peak $\mathcal{N}\left(\alpha_{*}\right)$ reaches the maximum value $N_{\text {max }}=0.1764$.

$\alpha>\alpha_{\max }(N)$, there is no intersection.

In Fig. 40, we have ploted $\alpha_{\min }$ and $\alpha_{\max }$ as a function of $N$. Let us mention some characteristic values. For $N=N_{\max }=0.1764$ we have $\alpha_{\min }=\alpha_{\max }=\alpha_{*}=$ 5.012. On the other hand, we find that $\alpha_{\min }=0$ for $N=N_{0}=0.1297$. Therefore, when $N>N_{0}$, the caloric curve is made exclusively of equilibrium states with $\alpha>0$ (positive chemical potential). Apart from this property, the value $N_{0}=0.1297$ does not seem to play a special role in the problem. 


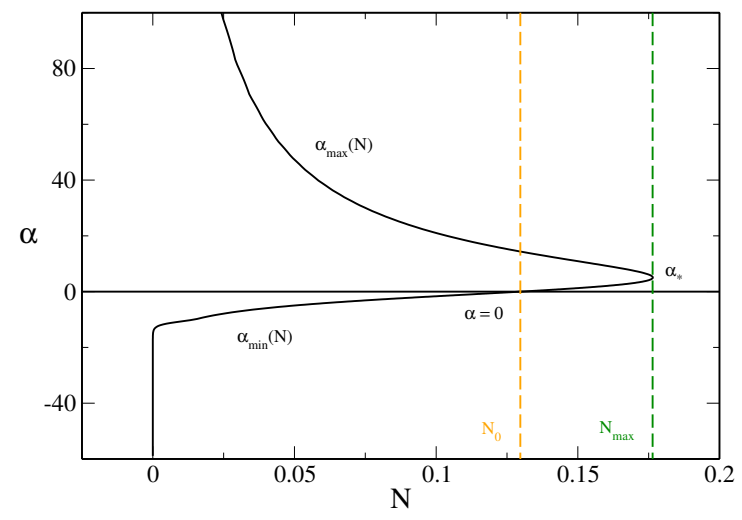

FIG. 40: Evolution of $\alpha_{\min }$ and $\alpha_{\max }$ with $N$. We find that $\alpha_{\min } \simeq-6.28+0.90 \ln N$ and $\alpha_{\max } \sim 2.40 / N$ for $N \rightarrow 0$.

\section{Relation with the caloric curves for different values of $N$}

We are now ready to discuss the relation between the topological properties of the curves $N_{\alpha}\left(\Phi_{0}\right)$ and the caloric curves $\eta(\Lambda)$ analyzed in Sec. V

$$
\text { a. } \quad N<N_{S}^{\prime}
$$

Let us consider the case $N<N_{S}^{\prime}=0.128$ (for illustration we take $N=0.1$ ).

We first consider the possible intersections between the curve $N_{\alpha}\left(\Phi_{0}\right)$ and the line level $N$ when $\alpha \leq \alpha_{*}$. As we shall see, this range of $\alpha$ is associated with the left part of the main branch + the "hot spiral" corresponding to the strongly relativistic gas.

For $\alpha<\alpha_{\min }(N)$, there is no intersection. For $\alpha$ just above $\alpha_{\min }(N)$, two intersections appear (see Fig. 41). If we keep increasing $\alpha$, we successively find more and more intersections, then less and less intersections, as the oscillations of the curve $N_{\alpha}\left(\Phi_{0}\right)$ traverse the line level $N$. For even larger values of $\alpha$, i.e. $\alpha>\alpha_{1}^{(\mathrm{H})}$, the oscillations of the curve $N_{\alpha}\left(\Phi_{0}\right)$ have passed above the line level $N$ so there is only one (relevant) intersection. We can see these different intersections, as a function of $\alpha$, in Figs. 42 and 43 (left side). Each set of intersections, as we vary $\alpha$, defines a branch $B_{i}^{(\mathrm{H})}=\left\{\Lambda_{i}(\alpha), \eta_{i}(\alpha)\right\}$ of the caloric curve $\eta(\Lambda): B_{1}^{(\mathrm{H})}$ is the branch corresponding to the first intersections (black), $B_{2}^{(\mathrm{H})}$ is the branch corresponding to the second intersections (red), etc. The ensemble of these branches forms the left part of the main branch and the "hot spiral". We see in Figs. 42 and 43 (left side) that the typical values of $\Phi_{0}$ and $T_{0}$ are large so the system is strongly relativistic on this part of the caloric curve. By plotting these branches in different colors on the density contrast versus energy curve of Fig. 4 or on the caloric curve of Fig. 35 , and considering the hot spiral, we observe that two successive branches merge at a

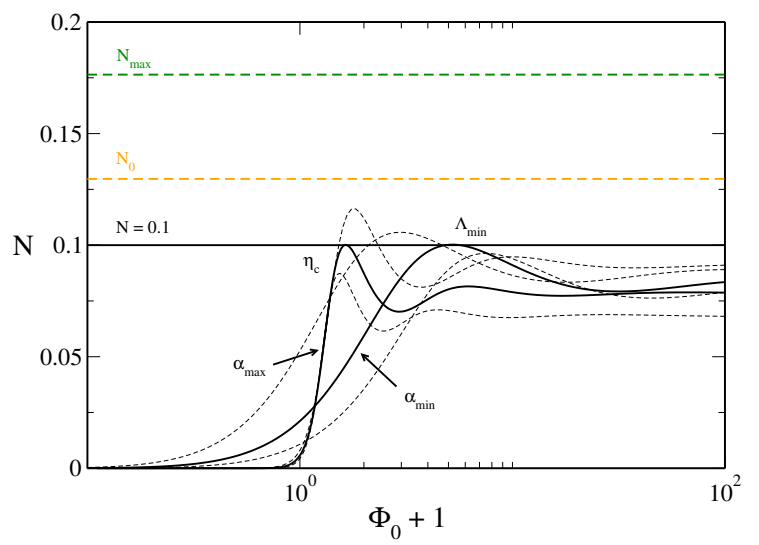

FIG. 41: (i) Appearance of two intersections when $\alpha$ passes above $\alpha_{\min }$ (the full line corresponds to $\alpha=\alpha_{\min }$ and the dashed lines correspond to values of $\alpha$ slightly below or above $\left.\alpha_{\min }\right)$. They are associated with the first turning point of energy $\Lambda_{\min }$ of the hot spiral ( $\Phi_{0}$ large). (ii) Disappearance of two intersections when $\alpha$ passes above $\alpha_{\max }$ (the full line corresponds to $\alpha=\alpha_{\max }$ and the dashed lines correspond to values of $\alpha$ slightly above or below $\alpha_{\max }$ ). They are associated with the first turning point of temperature $\eta_{c}$ of the cold spiral ( $\Phi_{0}$ small).

turning point of energy (we do not have a mathematical proof for that). In particular, the branches $B_{1}^{(\mathrm{H})}$ and $B_{2}^{(\mathrm{H})}$ corresponding to the first two intersections (plotted in black and red) merge, for $\alpha=\alpha_{\min }(N)$, at the critical point $\Lambda_{\min }$ corresponding to the maximum energy. Similarly, the branches $B_{2}^{(\mathrm{H})}$ and $B_{3}^{(\mathrm{H})}$ corresponding to the second and third intersections (red and green) merge at the second turning point of energy, and so on... As a result, for $\alpha \leq \alpha_{*}$, the ensemble of the first intersections $B_{1}^{(\mathrm{H})}$ forms the left part of the main branch of the caloric curve and the ensemble of the subsequent intersections $B_{2}^{(\mathrm{H})}, B_{3}^{(\mathrm{H})} \ldots$ form the "hot spiral".

We now consider the possible intersections between the curve $N_{\alpha}\left(\Phi_{0}\right)$ and the line level $N$ when $\alpha \geq \alpha_{*}$. As we shall see, this range of $\alpha$ is associated with the right part of the main branch + the "cold spiral" corresponding to the weakly relativistic gas. To make the discussion symmetric with respect to the previous one, we start from $\alpha \rightarrow+\infty$ and progressively decrease its value.

For $\alpha>\alpha_{\max }(N)$, there is no intersection. For $\alpha$ just below $\alpha_{\max }(N)$, two intersections appear (see Fig. 41). If we keep decreasing $\alpha$, we successively find more and more intersections, then less and less intersections, as the oscillations of the curve $N_{\alpha}\left(\Phi_{0}\right)$ traverse the line level $N$. For even lower values of $\alpha$, i.e. $\alpha<\alpha_{1}^{(\mathrm{C})}$, the oscillations of the curve $N_{\alpha}\left(\Phi_{0}\right)$ have passed above the line level $N$ so there is only one (relevant) intersection. We can see these different intersections, as a function of $\alpha$, in Figs. 42 and 43 (right side). Each set of intersections, as we vary $\alpha$, defines a branch $B_{i}^{(\mathrm{C})}=\left\{\Lambda_{i}(\alpha), \eta_{i}(\alpha)\right\}$ of the 


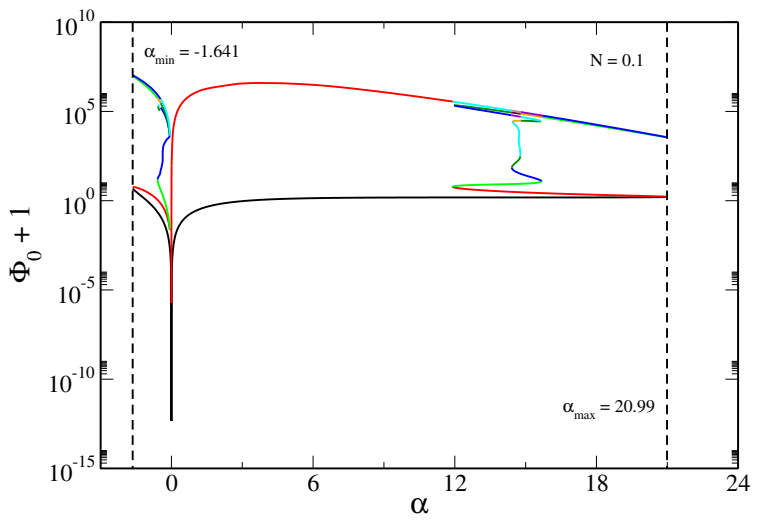

FIG. 42: Central value of the gravitational potential $\left(\Phi_{0}\right)_{i=1,2, \ldots}$, corresponding to the different intersections between the curve $N_{\alpha}\left(\Phi_{0}\right)$ and the line level $N$ (here $N=0.1$ ), as a function of $\alpha$. These curves clearly displays the bound $\alpha_{\min }$ and $\alpha_{\max }$ between which equilibrium states exist. Each intersection is plotted with a different color, the first one corresponding to the black curve, the second to the red curve, the third to the green curve etc. We are essentially interested in the first two intersections because the other ones correspond to unstable equilibrium states.

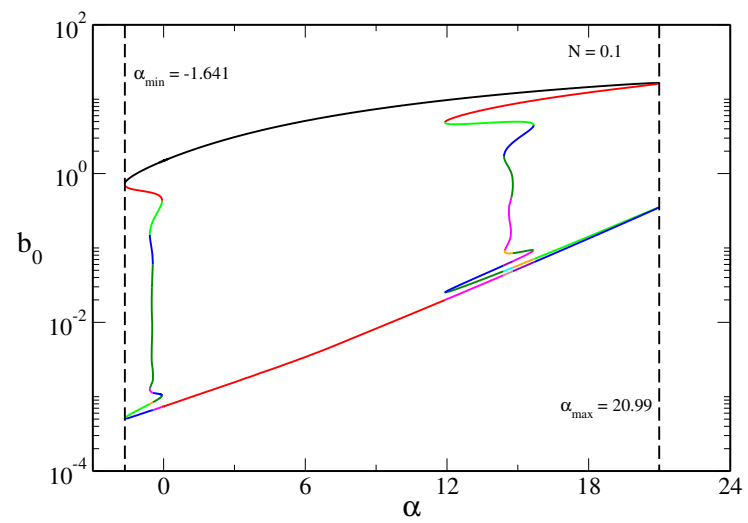

FIG. 43: Same as Fig. 42 except that we have plotted the inverse central temperature $b_{0}$ instead of the central potential $\Phi_{0}$ for a better visualization.

caloric curve $\eta(\Lambda): B_{1}^{(\mathrm{C})}$ is the branch corresponding to the first intersections (black), $B_{2}^{(\mathrm{C})}$ is the branch corresponding to the second intersections (red), etc. The ensemble of these branches forms the right part of the main branch and the "cold spiral". We see in Figs. 42 and 43 (right side) that the typical values of $\Phi_{0}$ and $T_{0}$ are small so the system is weakly relativistic on this part of the caloric curve. By plotting these branches in different colors on the density contrast versus temperature curve of Fig. 5 or on the caloric curve of Fig. 35 and considering the cold spiral, we observe that two successive branches merge at a turning point of temperature (we do not have a mathematical proof for that). In particular, the branches $B_{1}^{(\mathrm{C})}$ and $B_{2}^{(\mathrm{C})}$ corresponding to the first two intersections (plotted in black and red) merge, for $\alpha=\alpha_{\max }(N)$, at the critical point $\eta_{c}$ corresponding to the minimum temperature. Similarly, the branches $B_{2}^{(\mathrm{C})}$ and $B_{3}^{(\mathrm{C})}$ corresponding to the second and third intersections (red and green) merge at the second turning point of temperature, and so on... As a result, for $\alpha \geq \alpha_{*}$, the ensemble of the first intersections $B_{1}^{(\mathrm{C})}$ forms the right part of the main branch of the caloric curve and the ensemble of the secondary intersections $B_{2}^{(\mathrm{C})}, B_{3}^{(\mathrm{C})} \ldots$ form the "cold spiral".

We can make the following comments:

(i) The roles of $\Lambda$ and $\eta$ are reversed for the hot and cold spirals. For the hot spiral, the first two branches $B_{1}^{(\mathrm{H})}$ and $B_{2}^{(\mathrm{H})}$ merge at the first turning point of energy $\Lambda_{\min }$. By contrast, for the cold spiral, the first two branches $B_{1}^{(\mathrm{C})}$ and $B_{2}^{(\mathrm{C})}$ merge at the first turning point of temperature $\eta_{c}$.

(ii) The branch $B_{1}^{(\mathrm{H})}$ corresponding to the ensemble of the first intersections for $\alpha_{\min } \leq \alpha \leq \alpha_{*}$ forms the left part of the main branch of the caloric curve $\eta(\Lambda)$ while the branch $B_{1}^{(\mathrm{C})}$ corresponding to the ensemble of the first intersections for $\alpha_{*} \leq \alpha \leq \alpha_{\max }$ forms the right part of the main branch of the caloric curve $\eta(\Lambda)$. They are represented in black in Fig. 35. These two branches meet at $\alpha=\alpha_{*}$. We have numerically observed, however, that $\alpha_{*}$ does not correspond to the point at which the density contrast is minimum (see Figs. 4 and 5 ).

(iii) Only the lower part of Fig. 42 and only the upper part of Fig. 43 are "relevant" in the sense explained in footnote 51 . We note that a part of the second intersection (plotted in red) lies in the irrelevant region. This corresponds to the dashed red curves that superimpose the main (black) branch of the caloric curve on Fig. 35 (we also see this irrelevant red branch on the right side of Figs. 4 and 57. The corresponding energy density profiles coincide except at the very center where the irrelevant (unstable) solution has a very high density (see Fig. 36).

Remark: We have seen [see comment (i) above] that the maximum of the curve $N_{\alpha}\left(\Phi_{0}\right)$ with $\alpha<\alpha_{*}$ determines the point $\Lambda_{\min }(N)$ of the caloric curve $\eta(\Lambda)$ corresponding to $N=\mathcal{N}(\alpha)$. Antisymmetrically, the maximum of the curve $N_{\alpha}\left(\Phi_{0}\right)$ with $\alpha>\alpha_{*}$ determines the point $\eta_{c}(N)$ on the caloric curve $\eta(\Lambda)$ corresponding to $N=\mathcal{N}(\alpha)$. Therefore, it is very easy to obtain the curves $\Lambda_{\min }(N)$ and $\eta_{c}(N)$ plotted in Figs. 22 and 23 . For each value of $\alpha$, we determine the values of $N, \eta$ and $\Lambda$ corresponding to the maximum of the curve $N_{\alpha}\left(\Phi_{0}\right)$. For $\alpha<\alpha_{*}$, they determine $N(\alpha)$ and $\Lambda_{\min }(\alpha)$. For $\alpha>\alpha_{*}$, they determine $N(\alpha)$ and $\eta_{c}(\alpha)$. By running $\alpha$ from $-\infty$ to $\alpha_{*}$ we obtain the curve $\Lambda_{\min }(N)$. On the other hand, by running $\alpha$ from $\alpha_{*}$ to $+\infty$ we obtain the curve $\eta_{c}(N)$. Unfortunately, it does not seem possible to obtain the values of $\eta_{\min }$ and $\Lambda_{c}$ by a simple graphical construction based on the curve $N_{\alpha}\left(\Phi_{0}\right)$. Therefore, the curves $\eta_{\min }(N)$ and $\Lambda_{c}(N)$ plotted in Figs. 22 and 23 must 
be obtained by plotting the caloric curve $\eta(\Lambda)$ for any value of $N$ and determining the values of $\eta_{\min }$ and $\Lambda_{c}$ "by hand" (in practice numerically) from these curves.

$$
\text { b. } \quad N_{S}^{\prime}<N<N_{S}
$$

Let us now consider the case $N_{S}^{\prime}<N<N_{S}$. We recall that $N_{S}^{\prime}$ corresponds to the first minimum of $N_{\alpha_{*}}\left(\Phi_{0}\right)$. The difference with the previous case is that the second and third intersections can never merge. As a result, the spirals will not be complete. They will be amputed (truncated) and touch each other as shown in Fig. 12.

$$
\text { c. } \quad N_{S}<N<N_{\max }
$$

Let us finally consider the case $N_{S}<N<N_{\max }$. We recall that $N_{S}$ corresponds to the second maximum of $N_{\alpha_{*}}\left(\Phi_{0}\right)$. The difference with the previous case is that there can be at most two intersections between the curve $N\left(\Phi_{0}\right)$ and the line level $N$. As a result there is no spiralling behavior. This is why the caloric curve $\eta(\Lambda)$ looks like a loop resembling the symbol $\infty$ as in Fig. 13 .
[1] P.H. Chavanis, preprint

[2] G. Alberti, P.H. Chavanis, arXiv:1808.01007

[3] P.H. Chavanis, preprint

[4] P.H. Chavanis, Phys. Rev. D 84, 043531 (2011)

[5] S. Chandrasekhar, Principles of Stellar Dynamics (University of Chicago Press, 1942)

[6] J.H. Jeans, Mon. Not. R. Astron. Soc. 74, 109 (1913)

[7] J.H. Jeans, Mon. Not. R. Astron. Soc. 76, 552 (1916)

[8] J.H. Jeans, Problems of cosmogony and stellar dynamics (Cambridge University Press, 1919)

[9] J.H. Jeans, Astronomy and Cosmogony (Cambridge University Press, 1929)

[10] A.S. Eddington, Stellar movements and the structure of the Universe (MacMillan, London, 1914)

[11] C.V.L. Charlier, Lund Meddelanden 28, 16 (1917)

[12] K. Schwarzschild, Seeliger Festschrift, P. 94 (1924)

[13] S. Rosseland, Mon. Not. R. Astron. Soc., 88, 208 (1928)

[14] W.M. Smart, Stellar Dynamics (Cambridge University Press, 1938)

[15] V.A. Ambartsumian, Ann. Leningrad State Univ. 22, 19 (1938)

[16] H. Mineur, Ann. Astrophys. 2, 1 (1939)

[17] L. Spitzer, Mon. Not. R. Astron. Soc. 100, 396 (1940)

[18] J.H. Jeans, Mon. Not. R. Astron. Soc. 76, 70 (1915)

[19] A.A. Vlasov, Zh. Eksp. i Teor. Fiz. 8, 291 (1938)

[20] M. Hénon, Astron. Astrophys. 114, 211 (1982)

[21] R. Emden, Gaskugeln (Leipzig, 1907)

[22] S. Chandrasekhar, An Introduction to the Theory of Stellar Structure (Dover, New York, 1939)

[23] A.S. Eddington, Mon. Not. R. Astron. Soc. 75, 366 (1915)

[24] A.S. Eddington, Mon. Not. R. Astron. Soc. 76, 572 (1916)

[25] O. Heckmann and H. Siedentopf, Zs. f. Ap. 1, 67 (1930)

[26] R.H. Dicke, Astron. J. 48, 108 (1939)

[27] E. Zöllner, Über die stabilität kosmischer Massen, Leipzig, (1871)

[28] A. Ritter, Wiedemann's Annalen 16, 166 (1882)

[29] R. Woolley, Mon. Not. R. Astron. Soc. 114, 191 (1954)

[30] R.W. Michie, Mon. Not. R. Astron. Soc. 125, 127 (1963)

[31] I. King, Astron. J. 70, 376 (1965)

[32] S. Chandrasekhar, Astrophys. J. 97, 255 (1943)

[33] S. Chandrasekhar, Astrophys. J. 97, 263 (1943)

[34] S. Chandrasekhar, Astrophys. J. 98, 54 (1943)

[35] P.-H. Chavanis, Astron. Astrophys. 556, A93 (2013)

[36] M.L. White, Astrophys. J. 109, 159 (1949)
[37] L. Spitzer, R. Härm, Astrophys. J. 127, 544 (1958)

[38] S. von Hoerner, Zeit. Astr. 44, 221 (1958)

[39] I. King, Astron. J. 63, 109 (1958)

[40] I. King, Astron. J. 63, 114 (1958)

[41] I. King, Astron. J. 63, 465 (1958)

[42] I. King, Astron. J. 64, 351 (1959)

[43] I. King, Astron. J. 65, 122 (1960)

[44] I.R. King, Astron. J. 71, 64 (1966)

[45] M. Hénon, Ann. Astrophys. 23, 668 (1960)

[46] M. Hénon, Ann. Astrophys. 24, 369 (1961)

[47] M. Hénon, Ann. Astrophys. 28, 62 (1965)

[48] M. Hénon, Astron. Astrophys. 2, 151 (1969)

[49] R.W. Michie, Astrophys. J. 133, 781 (1961)

[50] R.W. Michie, P.H. Bodenheimer, Mon. Not. R. Astron. Soc. 126, 269 (1963)

[51] R.W. Michie, Mon. Not. R. Astron. Soc. 126, 331 (1963)

[52] R.H. Miller, E.N. Parker, Astrophys. J. 140, 50 (1964)

[53] L. Spitzer, W.C. Saslaw, Astrophys. J. 143, 400 (1966)

[54] K.F. Ogorodnikov, Sov. Astron. 1, 748 (1957)

[55] K.F. Ogorodnikov, Sov. Astron. 1, 787 (1957)

[56] D. Lynden-Bell, Mon. Not. R. Astron. Soc. 136, 101 (1967)

[57] V.A. Antonov, Vest. Leningr. Gos. Univ. 7, 135 (1962)

[58] D. Lynden-Bell and R. Wood, Mon. Not. R. Astron. Soc. 138, 495 (1968)

[59] D. Lynden-Bell, Bull. Astron. 3, 305 (1968)

[60] S. von Hoerner, Zeit. Astr. 50, 184 (1960)

[61] S. von Hoerner, Zeit. Astr. 57, 47 (1963)

[62] S. von Hoerner, Bull. Astron. (Paris) 3, 147 (1968)

[63] S.J. Aarseth, Mon. Not. R. Astron. Soc. 132, 35 (1966)

[64] H. Poincaré, Acta Math. 7, 259 (1885)

[65] R.A. Lyttleton, Stability of Rotating Liquid Masses (Cambridge University Press, 1953)

[66] P. Ledoux, in Handbook Der Physik, Ed. S. Flügge, Vol. 51 (Springer, Berlin, 1958)

[67] R. Ebert, Z. Astrophys. 37, 217 (1955)

[68] R. Ebert, Z. Astrophys. 42, 263 (1957)

[69] W.B. Bonnor, Mon. Not. R. Astron. Soc. 116, 351 (1956)

[70] W.H. McCrea, Mon. Not. R. Astron. Soc. 117, 562 (1957)

[71] M. Schönberg, S. Chandrasekhar, Astrophys. J. 96, 161 (1942)

[72] W. Thirring, Z. Physik 235, 339 (1970)

[73] G. Horwitz, J. Katz, Astrophys. J. 211, 226 (1977)

[74] G. Horwitz, J. Katz, Astrophys. J. 222, 941 (1978) 
[75] D. Lynden-Bell, N. Sanitt, Mon. Not. Roy. Astr. Soc. 143, 167 (1969)

[76] J. Katz, Mon. Not. R. Astron. Soc. 183, 765 (1978)

[77] J. Katz, Mon. Not. R. Astron. Soc. 189, 817 (1979)

[78] M. Lecar, J. Katz, Astrophys. J. 243, 983 (1981)

[79] T. Padmanabhan, Phys. Rep. 188, 285 (1990)

[80] T. Padmanabhan, Astrophys. J. Supp. 71, 651 (1989)

[81] S. Yabushita, Mon. Not. R. Astron. Soc. 140, 109 (1968)

[82] S. Yabushita, Mon. Not. R. Astron. Soc. 171, 85 (1975)

[83] L.G. Taff, H.M. van Horn, Mon. Not. R. Astron. Soc. 167, 427 (1974)

[84] L.G. Taff, H.M. van Horn, Astrophys. J. 197, L23 (1975)

[85] Y. Nakada, Publ. Astron. Soc. Japan 30, 57 (1978)

[86] I. Hachisu, D. Sugimoto, Prog. Theor. Phys. 60, 123 (1978)

[87] I. Hachisu, Y. Nakada, K. Nomoto, D. Sugimoto, Prog. Theor. Phys. 60, 393 (1978)

[88] S. Inagaki, Publ. Astron. Soc. Japan 32, 213 (1980)

[89] P.H. Chavanis, Astron. Astrophys. 381, 340 (2002)

[90] P.H. Chavanis, Astron. Astrophys. 401, 15 (2003)

[91] P.H. Chavanis, C. Rosier, C. Sire, Phys. Rev. E 66, 036105 (2002)

[92] C. Sire, P.H. Chavanis, Phys. Rev. E 66, 046133 (2002)

[93] C. Sire, P.H. Chavanis, Phys. Rev. E 69, 066109 (2004)

[94] P.H. Chavanis, C. Sire, Phys. Rev. E 70, 026115 (2004)

[95] P.H. Chavanis, C. Sire, Phys. Rev. E 73, 066103 (2006)

[96] P.H. Chavanis, C. Sire, Phys. Rev. E 73, 066104 (2006)

[97] P.H. Chavanis, C. Sire, Phys. Rev. E 83, 031131 (2011)

[98] J. Katz, I. Okamoto, Mon. Not. R. Astron. Soc. 317, $163(2000)$

[99] J. Katz, Found. Phys. 33, 223 (2003)

[100] P.H. Chavanis, Astron. Astrophys. 432, 117 (2005)

[101] P.H. Chavanis, Int. J. Mod. Phys. B 20, 3113 (2006)

[102] H.J. de Vega, N. Sanchez, Nucl. Phys. B 625, 409 (2002)

[103] H.J. de Vega, N. Sanchez, Nucl. Phys. B 625, 460 (2002)

[104] H.J. de Vega, N. Sanchez, F. Combes, Phys. Rev. D 54, 6008 (1996)

[105] B. Semelin, H.J. de Vega, N. Sanchez, F. Combes, Phys. Rev. D 59, 125021 (1999)

[106] B. Semelin, N. Sanchez, H.J. de Vega, Phys. Rev. D 63, 084005 (2001)

[107] J. Messer, H. Spohn, J. Stat. Phys. 29, 561 (1982)

[108] M. Kiessling, J. Stat. Phys. 55, 203 (1989)

[109] A. Gabrielli, M. Joyce, B. Marcos, F. Sicard, J. Stat. Phys. 141, 970 (2010)

[110] M. Champion, A. Alastuey, T. Dauxois, S. Ruffo, J. Phys. A 47, 225001 (2014)

[111] J. Katz, G. Horwitz, A. Dekel, Astrophys. J. 223, 299 (1978)

[112] J. Katz, Mon. Not. R. Astron. Soc. 190, 497 (1980)

[113] L. Casetti, C. Nardini, Phys. Rev. E 85, 061105 (2012)

[114] P.H. Chavanis, M. Lemou, F. Méhats, Phys. Rev. D 91, 063531 (2015)

[115] R.B. Larson, Mon. Not. R. Astron. Soc. 147, 323 (1970)

[116] R.B. Larson, Mon. Not. R. Astron. Soc. 150, 93 (1970)

[117] M. Hénon, Astrophys. Space Sci. 13, 284 (1971)

[118] S.J. Aarseth, Astron. Astrophys. 35, 237 (1974)

[119] D. Lynden-Bell, P.P. Eggleton, Mon. Not. R. Astron. Soc. 191, 483 (1980)

[120] H. Cohn, Astrophys. J. 242, 765 (1980)

[121] S. Inagaki, D. Lynden-Bell, Mon. Not. R. Astron. Soc. 205, 913 (1983)

[122] D. Sugimoto, E. Bettwieser, Mon. Not. R. Astron. Soc.
204, 19 (1983)

[123] D. Heggie, N. Ramamani, Mon. Not. R. Astron. Soc. 237, 757 (1989)

[124] J.P. Doremus, M.R. Feix, G. Baumann, Phys. Rev. Lett. 26, 725 (1971)

[125] J.P. Doremus, M.R. Feix, G. Baumann, Astron. Astrophys. 29, 401 (1973)

[126] D. Gillon, M. Cantus, J.P. Doremus, G. Baumann, Astron. Astrophys. 50, 467 (1976)

[127] J.F. Sygnet, G. Des Forets, M. Lachieze-Rey, R. Pellat, Astrophys. J. 276, 737 (1984)

[128] H. Kandrup, J.F. Sygnet, Astrophys. J. 298, 27 (1985)

[129] H. Kandrup, Astrophys. J. 370, 312 (1991)

[130] J.R. Ipser, G. Horwitz, Astrophys. J. 232, 863 (1979)

[131] A. Campa, P.H. Chavanis, J. Stat. Mech. 06, 06001 (2010)

[132] Y.B. Zel'dovich, M.A. Podurets, Soviet Astron. - AJ 9, 742 (1966)

[133] E.D. Fackerell, Ph.D. thesis (University of Sydney, 1966)

[134] N.A. Dmitriev, S.A. Kholin, Voprosy kosmogonii 9, 254 (1963)

[135] J.A. Wheeler, American Scientist 56, 1 (1968)

[136] J.A. Wheeler, The American Scholar 37, 248 (1968)

[137] A. Ewing, Sci. News Lett. 85, 39 (1964)

[138] A. Rosenfeld, Life Magazine 24, 11 (1964)

[139] C.A.R. Herdeiro, J.P.S. Lemos, arXiv:1811.06587

[140] J.R. Ipser, Astrophys. J. 158, 17 (1969)

[141] J.R. Ipser, K.S. Thorne, Astrophys. J. 154, 251 (1968)

[142] J.R. Ipser, Astrophys. J. 156, 509 (1969)

[143] J. Katz, G. Horwitz, M. Klapisch, Astrophys. J. 199, 307 (1975)

[144] K.G. Suffern, E.D. Fackerell, Astrophys. J. 203, 477 (1976)

[145] E.D. Fackerell, K.G. Suffern, Aust. J. Phys. 29, 311 (1976)

[146] M. Merafina, R. Ruffini, Astron. Astrophys. 221, 4 (1989)

[147] M. Merafina, R. Ruffini, Europhys. Lett. 9, 621 (1989)

[148] M. Merafina, R. Ruffini, Astron. Astrophys. 227, 415 (1990)

[149] G.S. Bisnovatyi-Kogan, M. Merafina, R. Ruffini, E. Vesperini, Astrophys. J. 414, 187 (1993)

[150] G.S. Bisnovatyi-Kogan, M. Merafina, R. Ruffini, E. Vesperini, Astrophys. J. 500, 217 (1998)

[151] D. Fackerell, J. Ipser, K. Thorne, Comments Ap. and Space Phys. 1, 134 (1969)

[152] J. Katz, G. Horwitz, Astrophys. J. 33, 251 (1977)

[153] J. Katz, G. Horwitz, Astrophys. J. 194, 439 (1974)

[154] G. Horwitz, J. Katz, Astrophys. J. 223, 311 (1978)

[155] J.R. Ipser, Astrophys. J. 238, 1101 (1980)

[156] J.R. Ipser, Astrophys. J. 193, 463 (1974)

[157] S.L. Shapiro, S.A. Teukolsky, Astrophys. J. 298, 34 (1985)

[158] S.L. Shapiro, S.A. Teukolsky, Astrophys. J. 298, 58 (1985)

[159] S.L. Shapiro, S.A. Teukolsky, Astrophys. J. 292, L41 (1985)

[160] S.L. Shapiro, S.A. Teukolsky, Astrophys. J. 307, 575 (1986)

[161] S.L. Shapiro, S.A. Teukolsky, Phil. Trans. R. Soc. Lond. A 340, 365 (1992)

[162] R.D. Sorkin, R.M. Wald, Z.Z. Jiu, Gen. Relat. Grav. 13, 1127 (1981) 
[163] P.H. Chavanis, Astron. Astrophys. 483, 673 (2008)

[164] R.C. Tolman, Phys. Rev. 35, 904 (1930)

[165] J.R. Oppenheimer, G.M. Volkoff, Phys. Rev. 55, 374 (1939)

[166] P.H. Chavanis, Astron. Astrophys. 381, 709 (2002)

[167] Z. Roupas, Class. Quantum Grav. 32, 135023 (2015)

[168] N. Bilic, R.D. Viollier, Eur. Phys. J. C 11, 173 (1999)

[169] Z. Roupas, P.H. Chavanis, Class. Quant. Grav. 36, 065001 (2019)

[170] P.H. Chavanis, G. Alberti, arXiv

[171] O. Klein, Rev. Mod. Phys. 21, 531 (1949)

[172] C.W. Misner, H.S. Zapolsky, Phys. Rev. Lett. 12, 635 (1964)

[173] B.K. Harrison, Phys. Rev. 137, 1644 (1965)

[174] B.K. Harrison, K.S. Thorne, M. Wakano, J.A. Wheeler, Gravitation Theory and Gravitational Collapse, (Chicago University Press, Chicago, 1965)

[175] G.S. Bisnovatyi-Kogan, Ya. B. Zel'dovich, Astrofizika 5, $223(1969)$

[176] G.S. Bisnovatyi-Kogan, K.S. Thorne, Astrophys. J. 160, 875 (1970)

[177] Chandrasekhar S., 1972, "A limiting case of relativistic equilibrium" in General Relativity, papers in honour of J.L. Synge, Edited by L.O' Raifeartaigh (Oxford)

[178] S. Yabushita, Mon. Not. R. Astron. Soc. 165, 11 (1973)

[179] S. Yabushita, Mon. Not. R. Astron. Soc. 165, 17 (1973)

[180] S. Yabushita, Mon. Not. R. Astron. Soc. 167, 95 (1974)

[181] H.J. Schmidt, F. Homann, Gen. Rel. Grav. 32, 919 (2000)

[182] T. Banks, W. Fischler, A. Kashani-Poor, R. McNees, S. Paban, Class. Quant. Grav. 19, 4717 (2002)

[183] A. Pesci, Class. Quant. Grav. 24, 2283 (2007)

[184] Ya. B. Zel'dovich, Soviet Phys. JETP 14, 1143 (1962)

[185] Dynamics and thermodynamics of systems with long range interactions, edited by T. Dauxois, S. Ruffo, E. Arimondo, M. Wilkens, Lecture Notes in Physics 602, (Springer, 2002)

[186] A. Campa, T. Dauxois, S. Ruffo, Physics Reports 480, $57(2009)$

[187] A. Campa, T. Dauxois, D. Fanelli, S. Ruffo, Physics of long-range interacting systems (Oxford University Press, 2014)

[188] P.H. Chavanis, Astron. Astrophys. 451, 109 (2006)

[189] Z. Roupas, Class. Quantum Grav. 30, 115018 (2013)

[190] Z. Roupas, Class. Quantum Grav. 32, 119501 (2015)

[191] X. Fang, X. He, J. Jing, Eur. Phys. J. C 77, 893 (2017)

[192] S.R. Green, J.S. Schiffrin, R.M. Wald, Class. Quantum Grav. 31, 035023 (2014)

[193] H.A. Buchdahl, Phys. Rev. 116, 1027 (1959)

[194] Ya.B. Zel'dovich, Soviet Phys. JETP 15, 1158 (1962)

[195] M.V. Penston, Mon. Not. R. Astron. Soc. 144, 425 (1969)

[196] Y. Pomeau, M. Le Berre, P.H. Chavanis and B. Denet,
Eur. Phys. J. E 37, 26 (2014)

[197] P.H. Chavanis, M. Lemou, F. Méhats, Phys. Rev. D 92, 123527 (2015)

[198] P.H. Chavanis, arXiv:1810.08948

[199] P.H. Chavanis, arXiv:1905.08137

[200] S. Balberg, S.L. Shapiro, S. Inagaki, Astrophys. J. 568, $475(2002)$

[201] M.C. Sormani, G. Bertin, Astron. Astrophys. 552, 37 (2013)

[202] P.H. Chavanis, Y. Pomeau, M. Le Berre and B. Denet, arXiv

[203] P. Hertel and W. Thirring, Thermodynamic Instability of a System of Gravitating Fermions. In: H.P. Dürr (Ed.): Quanten und Felder (Brauschweig: Vieweg 1971)

[204] N. Bilic, R.D. Viollier, Phys. Lett. B 408, 75 (1997)

[205] P.H. Chavanis, Phys. Rev. E 65, 056123 (2002)

[206] Z. Roupas, arXiv:1809.04408

[207] Z. Roupas, Universe 5, 12 (2019)

[208] H. von Zeipel, C. R. 144, 361 (1907)

[209] H.C. Plummer, Mon. Not. R. Astron. Soc. 71, 460 (1911)

[210] Lord Kelvin, Math. Phys. Papers 3, 255 (1862)

[211] Lord Kelvin, Phil. Mag. 22, 287 (1887)

[212] J.H. Lane, Amer. J. Sci. 50, 57 (1869)

[213] A. Ritter, Wiedemann's Annalen 11, 338 (1880)

[214] A. Schuster, Brit. Assoc. Rept., P. 427 (1883)

[215] A.S. Eddington, Mon. Not. R. Astron. Soc. 74, 5 (1913)

[216] J. Binney, S. Tremaine, Galactic Dynamics (Princeton Series in Astrophysics, 1987)

[217] J.H. Jeans, Mon. Not. R. Astron. Soc. 76, 567 (1916)

[218] A.S. Eddington, The Internal Constitution of the Stars (Cambridge, 1926)

[219] A.S. Eddington, Mon. Not. R. Astron. Soc. 76, 525 (1916)

[220] L.D. Landau and E.M. Lifshitz, Statistical Physics (Pergamon Press, New York, 1958)

[221] W. Thirring, Gen. Rel. Grav. 1, 31 (1970)

[222] S. Chandrasekhar, Astrophys. J. 74, 81 (1931)

[223] D. ter Haar, Proc. Int. Conf. on Statistical Mechanics (Kyoto, 1968)

[224] P. Hertel and W. Thirring, Ann. Phys. 63, 520 (1971)

[225] E.B. Aronson, C.J. Hansen, Astrophys. J. 177, 145 (1972)

[226] N.G. van Kampen, Phys. Rev. 135, 362 (1964)

[227] R.D. Carlitz, Phys. Rev. D 5, 3231 (1972)

[228] J.D. Bekenstein, Phys. Rev. D 9, 3292 (1974)

[229] S. Hawking, Nature 248, 30 (1974)

[230] S. Hawking, Phys. Rev. D 13, 191 (1976)

[231] D. Lynden-Bell, R.M. Lynden-Bell, Mon. Not. R. Astron. Soc. 181, 405 (1977)

[232] D. Lynden-Bell, Physica A 263, 293 (1999) 